\title{
The Intersection of Theory and Application in Elucidating Pattern Formation in Developmental Biology
}

\author{
H. G. Othmer ${ }^{1 *}$, K. Painter ${ }^{2}$, D. Umulis ${ }^{3}$ and C. Xue ${ }^{4}$ \\ ${ }^{1}$ School of Mathematics and Digital Technology Center \\ University of Minnesota, Minneapolis, MN 55455 USA \\ 2 Department of Mathematics \\ Department of Mathematics and Maxwell Institute for Mathematical Sciences \\ Heriot-Watt University, Edinburgh, EH14 4AS, UK \\ ${ }^{3}$ Agricultural \& Biological Engineering \\ Purdue University, West Lafayette, IN USA 47907 USA \\ ${ }^{4}$ Mathematical Biosciences Institute \\ Ohio State University, Columbus, OH 43210 USA
}

\begin{abstract}
We discuss theoretical and experimental approaches to three distinct developmental systems that illustrate how theory can influence experimental work and vice-versa. The chosen systems - Drosophila melanogaster, bacterial pattern formation, and pigmentation patterns - illustrate the fundamental physical processes of signaling, growth and cell division, and cell movement involved in pattern formation and development. These systems exemplify the current state of theoretical and experimental understanding of how these processes produce the observed patterns, and illustrate how theoretical and experimental approaches can interact to lead to a better understanding of development. As John Bonner said long ago

'We have arrived at the stage where models are useful to suggest experiments, and the facts of the experiments in turn lead to new and improved models that suggest new experiments. By this rocking back and forth between the reality of experimental facts and the dream world of hypotheses, we can move slowly toward a satisfactory solution of the major problems of developmental biology.'
\end{abstract}

Key words: robustness, chemotaxis, bacterial patterns, animal coat markings AMS subject classification: 35Q80, 92B05

\footnotetext{
*Corresponding author. E-mail: othmer@math.umn.edu
} 


\section{Introduction}

The word pattern, which stems from the French patron and the Latin patronus, means variously a model or template (as in design), a regularly-repeating sequence of shapes, objects or states in time and/or space (in mathematics), or more colloquially, something to be emulated. What does it mean in biology? Often the term is used loosely to mean the variation of a structure or occurrence or characteristic in space and/or time. For example, the leopard spots and zebra stripes shown in Figure 1 show differing degrees of regularity, and animal coat markings in general usually do not exhibit precise symmetry properties, yet we refer to them as patterns. Similarly, the arrangement of hair follicles is referred to as a spatial pattern, even though its primary characteristic is a roughly equal distance in space between the 'units'. Obviously there are many levels at which what we call pattern occurs, from the population-level, as in the bacterial patterns described later, to the single organism level of animal coat markings (though many cells are involved here), to the single cell level in early Drosophila melanogaster development. Certainly the mechanisms that generate patterns at the different levels differ in detail, but some aspects are common: there must be a signal of some sort, and mechanisms for detecting that signal and acting on it. Of course what the signal is and what it means to act on the signal differs from case to case.

Skin pigmentation markings, such as the characteristic stripes of zebras or the spots of a leopard, provide one of the most common and elegant examples of biological patterns (see Figure 1). Many of the advantages that accrue from the display of markings are obvious: these include camouflage, warnings, like-recognition and mate attraction, yet a number of less apparent benefits have also been set forth. For instance, in the case of zebra stripes these include social interactions, thermoregulation and even protection from insects such as tsetse flies [193].

In the developmental context, the adult form of an organism is achieved by numerous repetitions of three basic developmental processes: (i) growth and cell division, (ii) morphogenetic movements of cells and, (iii) determination and molecular differentiation of cells. The developmental fate of either isolated cells or cells in a tissue is governed both by its current internal state and by its previous history. Pictorially one can view the instantaneous internal state as a point in a high dimensional space in which the coordinates are concentrations and other relevant factors, and the evolution of the state in time determines a curve, or better a tube, given the inevitable fluctuations in the state, in this state space. A cell is said to be determined when a particular developmental pathway has been singled out, whereas it is differentiated when the program for that pathway has been executed. At the molecular level, determination is generally viewed as either preparation for transcription, or actual transcription, of a particular battery of genes, whereas molecular differentiation involves the production of enzymes or structural proteins that alter the morphology and function of a cell. These processes are more or less tightly coupled, though there may be separate control of RNA processing, and hereafter we simply speak of differentiation.

\subsection{Fundamental mechanisms of pattern formation}

While significant advances in understanding the molecular components involved in signal transduction and gene expression for model systems such as Drosophila melanogaster have been made 

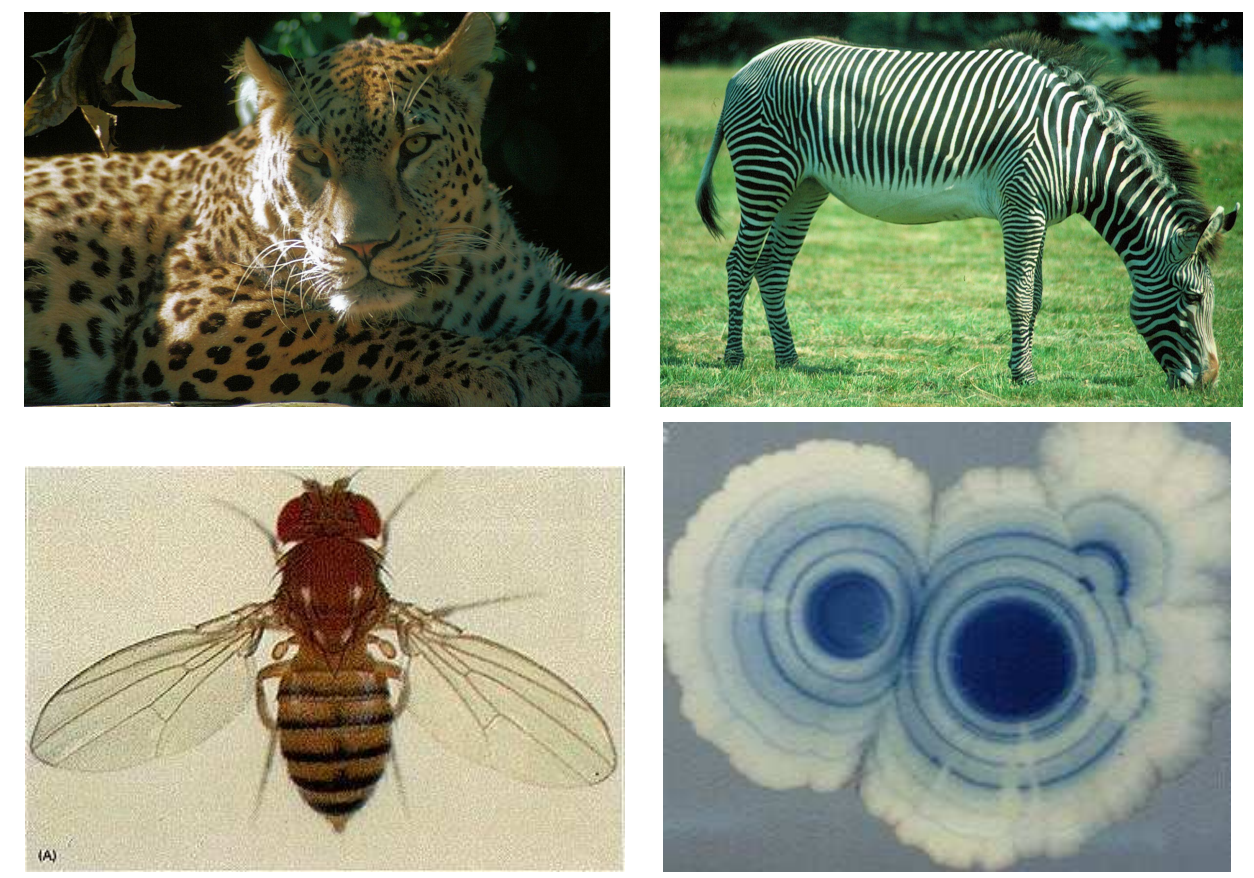

Figure 1: Patterning at various levels. Top: The leopard and the zebra. (left) Persian Leopard, (Right) Grevy's zebra, Bottom: The fruitfly Drosophila melanogaster (left) and patterns in E. coli colonies (right) (courtesy of J. Shapiro).

in the past forty years, the problem of pattern formation remains a major challenge. For some time following the discovery of the structure of DNA, the prevailing attitude was that the problem would be solved if one could understand how the information encoded in genes is translated into the patterns of synthesis that characterizes a given cell type. However, this was a faint hope, because the developmental fate of a cell in a multicellular system (which we will call a tissue) is determined not only by its genome, but also by its internal state, which in turn can depend on its spatial position within the tissue. The genes do not determine the detailed program of cellular activity, but rather, limit the scope of these activities. By setting bounds on the type of response that is possible for a cell in a given environment at a given stage of its development, the broad outline of cell development is set, but details remain a function of the cellular environment. Because gene transcription can be influenced by transcription factors in the cytoplasm, and because cells can exchange material either directly via gap junctions or indirectly via the extracellular space, intercellular communication can play a significant role in determining which part of the genetic code is transcribed at a particular point in space and time.

\subsubsection{Patterning controlled by prepatterns}

Morphogen-based models for pattern formation and regulation fall into two main classes: those with specialized regions that produce or degrade morphogens and those in which pattern emerges spontaneously in an aggregate of identical cells. Models of the former type are generally described as positional information models, but we call them prepattern models, since the next stage of 
patterning is predicated on the pattern established in a previous stage. The other main class has two subclasses, one in which the pattern has a high degree of regularity with respect to some reference frame, for example, the boundary of a tissue, whereas the other type is characterized by a more or less random reference point of the structures, but also a more or less uniform spacing between them. The canonical example of the first subclass is a Turing model, and an example of the second is the spacing of hair follicles [42].

While spatial differentiation can certainly occur in a single isolated cell such as the Drosophila egg, the difficult theoretical problems arise in tissues, because in order to produce position-dependent differentiation, each cell must evolve along the appropriate curve in state space so as to select the appropriate developmental pathway. Such space dependent differentiation or pattern formation can be thought of as resulting from the cellular response to an appropriate spatial pattern of control signals that establishes which genes are to be transcribed, which proteins produced, and what other changes of cellular state are to be made. These signals can be biochemical or mechanical, and may be the concentration of a critical substrate that produces changes in transcription, an extracellular diffusable ligand that produces downstream changes in response to changes in occupancy of receptors in the membrane, or some other cellular state variable. This primary pattern of signals, which we call a prepattern, may be inherited or externally-maintained, or it may arise spontaneously within the tissue. Development usually proceeds in stages, and the pattern in one stage often serves as the prepattern of the next, as will be seen in the context of Drosophila melanogaster. In Alan Turing's words 'Most of an organism, most of the time, is developing from one pattern into another, rather than from homogeneity into a pattern' [219].

The spatial distribution of extracellular state variables defines what we call the morphogenetic landscape to which the cells respond, and frequently alter by release of components into the extracellular space. When the signals in extracellular variables are diffusible molecules that affect the internal state in a concentration-dependent manner, they are called morphogens, a term coined by the British mathematician Turing [219]. More precisely, morphogens are defined as secreted signaling molecules that (i) are produced in a restricted portion of a tissue, (ii) are transported by diffusion [116], active transport, relay mechanisms, or other means to the remainder of the tissue [109], (iii) are detected by specific receptors or bind to regulatory regions of DNA, and (iv) initiate an intracellular signal transduction cascade that initiates or terminates the expression of target genes in a concentration-dependent manner. The concept of a morphogenetic landscape, usually described as a developmental field similar to the classical fields in physics, played a role throughout the early history of theoretical work in pattern formation [40]. When morphogens are involved, the morphogenetic landscape usually varies smoothly in space, but as the zebra exemplifies, and as will be seen later for Drosophila melanogaster, there is often a very sharp delineation between different elements of a pattern. Thus the response to an established prepattern may require conversion of a smoothly-varying extracellular signal into a step-like response via some downstream thresholding mechanism.

The early theories of developmental fields were synthesized into the theory of positional information by Wolpert [235]. Formally the theory posits that a cell must 'know' its position relative to other cells in order to adopt the correct developmental pathway, but of course what a cell 'knows' is determined by the information it extracts from the past and current signals received. Drosophila 
melanagaster is one of several model systems discussed later for which a number of morphogens have been identified, and for which many of the components of the signal transduction and gene control networks involved in patterning are known. However, less is known about how these networks produce the desired spatio-temporal pattern of gene expression.

\subsubsection{Spontaneous emergence of pattern}

To distinguish between the control mechanism underlying spontaneously-forming patterns that are fixed with respect to a boundary from those characterized by a more or less random location but regular spacing, consider a two-dimensional network of cells, each of which is coupled to its nearest neighbors by junctional membranes, and suppose that the network is effectively isolated from the remainder of the organism. In the second type of pattern, the spatial distribution of differentiated cells is not rigidly fixed relative to the boundary, but rather, the spacing between differentiated cells is approximately constant. Examples of this kind of pattern include the location of certain kinds of insect bristles [43], the spacing of hair follicles [42], the pattern of peripheral nerve connections in vertebrates, the eye where Notch-Delta signaling is involved [60,27] and in general, any pattern in which the absolute number and location of pattern elements is less important than the final density of these elements.

In contrast, the first type of pattern contains a fixed number of structures located at more or less fixed positions. Examples of this type abound: on many insects the sensory structures on the epidermis are arranged in a constant number of rows with a fixed number of structures in each row. Other examples are the over-all body segmentation pattern in arthropods and worms, the segmentation of internal structures such as vertebrate somites, and the synaptic connections of retinal ganglion cells in the midbrain tectum. In many of these examples the number of structures is constant over a wide range of total cell number and hence aggregate size, thereby reflecting a high degree of regulation with respect to the number of structures. Figuratively speaking, these systems behave as though the basic pattern is embedded in a rubber sheet and then stretched or compressed to accommodate to the overall size of the organism.

It is easy to construct a hypothetical control scheme for the second type of pattern. As an example, consider an insect bristle pattern and suppose that initiation of bristle formation occurs when the concentration of some essential substrate exceeds a threshold level. Further, suppose that every cell produces this substrate, that growth and maintenance of a bristle requires a constant supply of the substrate, and that the production rate in a single cell is insufficient to support a bristle. If all cells are initially below threshold, initiation will occur in randomly-located cells in the aggregate. If the cells are all coupled and the substrate can diffuse between cells, bristle formation in a given cell will deplete the substrate in surrounding cells and prevent formation of nearby bristles. Statistical analysis of the final density of structures that can be supported has been carried out and the results agree very well with observed densities, not only for bristle patterns but also for hair follicle patterns in several different species [76]. An alternate hypothesis that leads to similar patterns has it that each bristle, once initiated, emits an inhibitory substance that prevents formation of bristles nearby. At present one cannot decide between the depletion and inhibition models because the molecular basis of the control scheme is not yet known. In a related problem, Jung, et al. [100] 
show that the feather tract is initiated by a continuous stripe of sonic hedgehog and growth factor expression in the epithelium, which then separates into distinct feather primordia. They develop a complex model that involves a uniformly-distributed activator, an activator of primordium 'competence' that propagates across the field, and local activators and inhibitors whose production is initiated in primordia.

The random-initiation-site model is clearly inadequate for producing the precisely-ordered patterns of the first type, and the most interesting theory proposed for the generation of such patterns is due to Turing [219]. In Turing's model two or more chemical substances called morphogens react within each cell and diffuse between cells. All cells are assumed to be identical initially and cells at the boundary are closed, and thus the reaction-diffusion equations that describe the model have a solution that is spatially- uniform. What Turing showed is that this uniform state can be unstable to some non-uniform disturbances if the kinetic interactions and the diffusion constants are chosen appropriately. Such instabilities, which Turing called symmetry-breaking, can lead to either a steady or time- periodic nonuniform distribution of morphogens. The unstable wavelengths are fixed by the kinetic coefficients and the diffusivities, and therefore each unstable system has an intrinsic chemical wavelength. Thus identical systems will give rise to an identical distribution of morphogens and, via an appropriate interpretation mechanism, to an identical pattern of cell differentiation. Turing himself suggested that the model could account for the regular spacing of tentacles on Hydra and that it might be applicable to phyllotaxis. In the early sixties several biologists used the model to explain the pattern of sensory structures on the epidermis of certain insects. Since none of the morphogens were identified in any of the systems, the applicability of the model to these systems still remains in doubt.

More recently the existence of theoretically-predicted patterns has been demonstrated under carefully controlled experimental conditions in the "CIMA" (Chlorite-Iodide-Malonic AcidStarch) reaction, $[39,54,171]$. When all of the measured parameters are entered into the mathematical model derived from the known chemical reactions, excellent agreement is found between the predicted patterns and those observed experimentally [120]. These and further observations leave little doubt that interactions of reaction and diffusion can give rise to instabilities and result in well-defined spatial patterns ( $c f$. also [127]).

What remains in doubt is whether the mechanism is applicable to any biological systems. Two potential applications are the generation of feather bud tracts in birds and hair follicle patterns of mammals ( $c f$. also [87], this issue). In the former, Jung et al. [100] have identified a number of activators and inhibitors that are involved in feather bud formation and suggest that the patterning may involve a Turing mechanism, but this remains to be shown. In the latter Sick et al. [207] report experimental evidence that Wnt and its inhibitor Dkk function as an activator-inhibitor pair in the Turing sense for hair follicle formation. On the other hand, Mou et al. [142] report the additional activator-inhibitor coupling between Edar receptor and its inhibitor BMP in localizing follicles. The incorporation of these interactions along with further species into the actual network raises questions as to whether a two-component representation will be adequate in practice. The best that can be done at present is to withhold judgment until more concrete tests of its applicability are possible. 


\subsubsection{Spatial patterns in populations of mobile units}

In the previous discussion we focused on patterning in systems of immobile objects, whether a single cell or a network of connected cells. In these systems the spatial patterning requires mechanisms for distinguishing the states of adjacent cells or regions within a cell, and reactiondiffusion mechanisms have been widely-invoked in this context. However, when the functional units are mobile, movement of the units may play an essential role in pattern formation, which adds a potentially-important new pattern-generating mechanism. Self-organization at the population level gives rise to flocks of birds, schools of fish, herds of various mammals, and at a lower level, various types of aggregations in single-cell organisms. It is only recently that the importance of multicellular organization in prokaryotes, aside from examples such as Myxococcus xanthus, has been recognized [201]. It is now known that intercellular communication and coordination are widespread in prokaryotes, and many different classes of signaling molecules have been identified. Current interest in this phenomenon stems from the discovery of quorum-sensing molecules such as the $\mathrm{N}$-acyl homoserine lactones, and their possible role in biofilm formation [68]. Much simpler, and more tractable from the modeling standpoint, examples of pattern formation arise in $E$. coli, S. typhimurium, and B. subtilis colonies [34, 35, 201]. Pattern formation in E. coli, which we discuss in detail later, involves sensing of extracellular signals and alterations in the swimming behavior of individuals, as well as production of the attractant. However, spatial patterns can involve millions of cells, and heretofore modeling of them has been primarily phenomenological; details of the cell-level behavior were not incorporated into the equations that describe population-level behavior until recently.

Another example of self-organized pattern formation in unicellular organisms arises in the social amoeba Dictyostelium discoideum, which uses oscillatory signaling for aggregation [168]. These cells normally live in forest soil where they feed on bacteria [28], but when the food supply runs out amoebae express receptors that allow them to detect cAMP. They can also relay cAMP signals, and after about eight hours post-starvation, randomly located cells called pacemakers begin to emit cAMP periodically. Nearby cells detect the signal, move towards the cAMP source, and relay the cAMP signal to more distant cells. Eventually the entire population collects into mound-shaped aggregates, each containing up to $10^{5}$ cells. Mounds elongate to form slugs, which topple over and migrate over the substratum. At this stage cells also start to differentiate into prestalk and prespore cells, which occurs randomly in a mound. However, as a result of cell sorting and cAMP signaling, the prestalk cells end up in the anterior quarter of the slug, while the prespore cells occupy the remaining posterior part. When conditions are favorable, the slug tip is extended upwards, the anterior prestalk cells become immobilized in a central stalk tube, which the remaining cells use as a support for upward movement. When the stalk has reached a certain length the prespore cells mature into spores, which remain dormant until they are dispersed and find conditions favorable for growth. The autonomous production and relay of cAMP pulses by starving cells results in a very efficient process of chemotactic aggregation, and cAMP oscillations subsequently organize the transformation of mounds into slugs, the migration of slugs over the substratum, and the culmination into fruiting bodies. 


\subsection{The role of modeling in understanding patterning}

The theoretical papers by Turing [219] and Wolpert [235] provided a framework within which to formulate hypotheses about pattern formation and differential gene expression, but it was not until 1988, thirty six years after Turing's paper and nearly twenty years after Wolpert's, that the first morphogen was identified in a biological system. Driever and Nusslein-Volhard found that changing the level or distribution of bicoid mRNA (bcd) resulted in changes in the bicoid (Bcd) protein distribution and a subsequent shift of anterior structures that respond to Bcd in Drosophila $[59,58]$. In addition to Bcd, a number of other morphogens have been identified, including the bone morphogenetic proteins, members of the fibroblast growth factor (FGF) family, the epithelial growth factor (EGF) family, and additional members of the transforming growth factor- $\beta$ (TGF- $\beta$ ) family (reviewed in $[115,191]$ ). Many questions about the various steps in Bcd signaling remain twenty years after the discovery of Bcd as a morphogen, but they go beyond the identity of the molecular components involved.

Currently rapid progress on many questions in pattern formation can be made by integrating mathematical modeling with experimentation in order to understand the mechanisms of patterning in various systems. Over the past decade there has been an explosion in the number of papers that couple mathematical modeling with experimentation to understand the biology, determine biophysical parameters, and provide a platform for hypothesis testing. Mathematical, mechanistic-based models are thus serving as tools for biological discovery in new and exciting ways, and in many instances the mathematical models are leading the biology, rather than simply explaining existing observations. Mathematical models for Drosophila oogenesis, Bcd patterning, BMP-mediated patterning, planar cell polarity, EGF patterning, and segment polarity have all led to experiments that would have not have otherwise been carried out, and contributed greatly to our understanding of those systems [241, 77, 206, 3, 184]. Some of these will be discussed later.

Numerous phenomenological models for patterning were developed well before the discovery of the first morphogen in a biological system, and continue to have a substantial impact today. The most prominent example is the Turing model discussed earlier, which has served precisely as Popper held scientific theories should, i.e., a theory should be considered scientific if and only if it is falsifiable. More colloquially, it is said that 'all experiments ever do is ruin a good theory', and this is what should be expected when developing a mathematical model. If the modeling work is compelling enough that it leads to experiments, which may take years to complete, the data that emerge from this endeavor will increase our understanding of the underlying mechanisms, and may very well invalidate some components of the model.

The availability of experimental data for Bcd and other morphogens has led to a shift from predominantly phenomenological models to mechanism-based models, the goals of which are not only to explain the existing observations within a mechanistic framework, but to also be useful as tools for discovery by biologists, biochemists, and geneticists. Some examples of how mathematical models of development have been used in the past are as follows.

- Determine what theoretically-possible mechanism(s) may work. This involves phenomenological modeling or initial hypothesis testing [61, 138, 203, 224] . 
- Determine how the system works: Quantitatively measure transport and molecular mechanisms of pattern formation and include this information in the model [184, 82, 110, 83, 224, 200]. This also includes simulating aspects of patterning that are not easily observed such as morphogen dynamics [224, 97, 138, 3].

- Determine why the system works as it does: Identify mechanisms that confer reproducibility, insensitivity, scale-invariance, or other beneficial performance objectives [61, 227, 224, 82, 203, 190, 83, 50, 167, 223, 21, 81].

As a specific example, mathematical modeling has proved very helpful in uncovering mechanisms of bacterial pattern formation, as illustrated by the following.

- E.coli can sense and adapt to ligand concentrations spanning five orders of magnitude, and can detect a change in occupancy of the aspartate receptor Tar of as little as $0.1 \%$ [29]. The gain of the system, defined as the change in bias divided by the change in receptor occupancy, can be as high as 55 [197], and mathematical modeling has been integrated with experimentation to understand the source of the high gain. The model developed in [210] predicted that in the absence of cooperativity in signal transduction, a Hill coefficient of at least 11 for binding of $\mathrm{CheY}_{p}$ at the motor was needed to explain the observed gain of 3-6, and this was subsequently confirmed experimentally [44].

- When E. coli cells secrete a substance to which they also respond, complex patterns may form under different growth conditions ( $c f$. Section 4.). The hybrid cell-based model described later provides opportunities to investigate the importance of intracellular processes during pattern formation by tuning intracellular parameters. One example starts from a steady state of the Patlak-Keller-Segel system with random noise. Simulation in 1D reveals that a longer adaptation time leads to a larger aggregate size, while either no adaptation or instantaneous adaptation preclude aggregation. Therefore a finite time for adaptation to the signal is important for aggregate formation, which explains the experimental finding that adaptation-defective mutants cannot aggregate.

System-specific details on how theoretical and experimental work has interacted to illustrate Bonner's assertion are given in the remainder of the paper. In the following section we give a brief introduction to the mathematics and physics of pattern-forming processes, and discuss the modeling process in general terms. In Section 3. we treat patterning in Drosophila melanogaster, for which the morphogens are known and cell differentiation involves a prepattern: Bcd along the anterior-posterior axis and others along the DV axis. Here the primary processes are reaction and diffusion, since the movement of nuclei to the periphery of the egg is not taken into account in the model. Section 4. deals with bacterial patterning, where pattern arises spontaneously due to signaling and cell movement. Both hybrids of cell-based and continuum models, as well as pure continuum models are used here, and the spatial patterns that emerge are primitive forms of biofilms. In addition to cell-cell signaling, cell motility plays an essential role here. In Section 5. we discuss pigmentation patterns that occur in butterflies, fish and mammals. As in the bacterial 
patterns, both spontaneous pattern formation and cell movement are involved, but in these systems patterning occurs on a growing domain, and growth can modify the patterning process significantly.

\section{An overview of the mathematics and physics of pattern for- mation}

\subsection{The French flag problem}

In the simplest version of a prepattern model, either specialized source and sink cells located at the boundary of the developmental field maintain the concentration of the morphogen at appropriate fixed levels, or they produce the morphogen at a fixed rate. In the former case, absent degradation of the morphogen, a linear distribution can be established in a one-dimensional system of about 1 $\mathrm{mm}$ in length in the time that is normally available for commitment to differentiation [236, 51], and given fixed thresholds between different cell types, the tissue can be proportioned into any number of cell types in a perfectly scale-invariant way. However this scheme clearly has limitations, since the flux between source and sink in a one-dimensional system of length $\mathrm{L}$ scales as $1 / \mathrm{L}$, and thus the morphogen producing or consuming cells at the boundary must adjust production to adjust for the size of the system. For the latter case we introduce a simple version in which the flux at the boundary, rather than the concentration, is specified - generalizations will be discussed later in the context of Drosophila patterning. Suppose that a single morphogen is produced at the boundary of a one-dimensional system, diffuses through the region, and decays via a first-order reaction. The governing equations for the steady-state morphogen concentration $c$ are

$$
\begin{aligned}
D \frac{d^{2} c}{d x^{2}} & =\kappa c & & x \in(0, L) \\
-D \frac{d c}{d x} & =j & & x=0 \\
\frac{d c}{d x} & =0 & & x=L .
\end{aligned}
$$

In scaled coordinates this becomes

$$
\begin{aligned}
\frac{d^{2} c}{d \xi^{2}} & =\lambda^{2} c & & \xi \in(0,1) \\
-\frac{d c}{d \xi} & =J & & \xi=0 \\
\frac{d c}{d \xi} & =0 & & \xi=1,
\end{aligned}
$$

where

$$
\lambda^{2} \equiv \kappa L^{2} / D \quad \text { and } \quad J=j L / D
$$


The solution of this is

$$
c(\xi)=\frac{J}{\lambda}\left[\frac{e^{\lambda(2-\xi)}+e^{\lambda \xi}}{e^{2 \lambda}-1}\right] \equiv \frac{J}{\lambda} \phi(\xi)=\frac{j}{\sqrt{\kappa D}} \phi(\xi) .
$$

Thus the stationary distribution is characterized by one dimensionless parameter $\lambda$ that is the ratio of a diffusion time scale $\tau_{D} \equiv L^{2} / D$ and a kinetic time scale $\tau_{K} \equiv \kappa^{-1}$, and a dimensional parameter that is the ratio of the input flux $j$ to a characteristic velocity defined by the diffusion constant and the decay rate. The former enters in the shape function $\phi$ via the exponential terms and determines how rapidly the morphogen concentration decays in space: the larger $\lambda$ the more rapidly the solution decays from the value at the source. It is clear that for a fixed input flux $j$ both the amplitude and the shape of the morphogen distribution depend on $L$, and thus this simple mechanism does not suffice when significant variations in length occur in the developing system. More complex patterning driven by prepatterns can result when there are several specialized boundary regions. One example arises in vertebrate limb development, where specialized regions called the ZPA and the AER direct outgrowth and patterning [57]; others to be discussed later include dorsoventral (DV) patterning in Drosophila melanogaster and the localization of eyespots in butterfly wing markings.

\subsection{The standard Turing model}

Turing models involve at least two morphogens that interact chemically and diffuse passively in a network of identical cells [219]. Turing only considered periodic systems or closed surfaces, in which case no boundary conditions are needed. More generally, any system of reaction-diffusion equations in which the kinetic mechanism has certain structural features described later, and for which the boundary conditions are of the same type for all species, will be called a Turing system [56]. Under certain conditions described later, instabilities of a spatially-uniform stationary state may develop and lead to either a spatially non-uniform stationary state or to more complicated dynamical behavior. The resulting non-uniform distribution might in turn lead, via an unspecified 'interpretation' mechanism, to spatially nonuniform differentiation. Most analyses of Turing models only treat instabilities of uniform stationary states, since numerical analysis is generally required for more general stationary states. However, Turing himself recognized the biological unreality of this in stating that 'most of an organism, most of the time is developing from one pattern to another, rather than from homogeneity into a pattern' [219]. An excellent review of some of the background on pattern formation, particularly of two-component Turing systems, is given in [52] and applications of two component systems to biological problems is described in $[75,131,10]$.

In the following analysis we will assume that the temporal dynamics in a spatially uniform system are governed by the solution of a system of ordinary differential equations. Let $\Omega$ be a bounded region in $\Re^{q}, q \leq 3$ with a smooth boundary and outward normal $\mathbf{n}$. The simplest version 
of Turing's model for pattern formation is described by the system of reaction-diffusion equations

$$
\begin{aligned}
\frac{\partial c}{\partial t} & =D \nabla^{2} c+\bar{R}(c, \bar{p}) \quad \text { in } \Omega \\
\mathbf{n} \cdot D \nabla c & =0 \quad \text { on } \partial \Omega \\
c(r, 0) & =c_{0}(r),
\end{aligned}
$$

where $c=\left(c_{1}, c_{2}, \cdots, c_{m}\right)^{T}, D$ is usually assumed to be a constant diagonal matrix, and $\bar{R}$ is the reaction rate vector. We assume for the present that all $D_{i}>0$. The reaction term $\bar{R}(c, \bar{p})$ may also contain mass transfer with the surroundings, for if the system is thin in one dimension then averaging across that dimension leads to a system such as (2.3) with transfer terms [169].

Let $\omega^{-1}$ be a time scale characteristic of the reactions, let $L$ be a measure of the size of the system, and let $C_{i}$ be a reference concentration for the $i^{\text {th }}$ species. Suppose that the species are ordered so that $\max _{i}\left\{D_{i}\right\}=D_{1}$. Define the dimensionless quantities $u_{i}=c_{i} / C_{i}, \tau=\omega t$, $\theta^{i}=D_{i} / D_{1}, \nu=D_{1} / \omega L^{2}$, and $\zeta=r / L$, where $r \equiv\left(x_{1}, \cdots, x_{q}\right)$. The dimensionless governing equations are

$$
\begin{array}{ll}
\frac{\partial u}{\partial \tau}=\nu \mathcal{D} \nabla^{2} u+R(u, p) & \text { in } \Omega \\
\mathbf{n} \cdot \nabla u=0 & \text { on } \partial \Omega \\
u(\zeta, 0)=u_{0}(\zeta), &
\end{array}
$$

where $\mathcal{D}=\operatorname{diag}\left\{1, \theta^{2}, \ldots, \theta^{m}\right\}$ and $R(u, p)$ is the dimensionless form of $\bar{R}(u, p)$. It is easy to show from (2.4) that when the reaction term is absent, the solution relaxes exponentially to the average concentration set by the initial conditions. It follows that whenever the relaxation time for diffusion of each species is sufficiently short compared to that of the chemical reactions, the solution of the variational equation should relax to zero. The relaxation time of the kinetics relative to that of diffusion is given by $\nu=\omega^{-1} /\left(L^{2} / D_{1}\right)$, and one should expect that solutions of the nonlinear problem will also converge to spatially uniform solutions when $\nu>>1$. In particular, this should occur when $L$ is sufficiently small, which has been shown rigorously in $[162,45,7]$.

However, Turing showed that diffusion could destabilize a steady state that is stable in the absence of diffusion, and when this occurs one calls it a Turing or diffusive instability. In order to define this precisely we have to consider the variational system associated with (2.4). Suppose that $u^{s}$ is a time-independent solution of (2.4), and suppose that we linearize (2.4) around $u^{s}$. If we let $\xi=u-u^{s}$, then we obtain the variational system

$$
\begin{array}{r}
\frac{\partial \xi}{\partial \tau}=\nu \mathcal{D} \nabla^{2} \xi+K \xi \\
\mathbf{n} \cdot \nabla \xi=0 \\
\xi(\zeta, 0)=\xi_{0}(\zeta)
\end{array}
$$


By assuming solutions of the form $\xi=e^{\lambda \tau} \Psi$, we obtain the spectral problem

$$
\begin{array}{r}
\nu \mathcal{D} \nabla^{2} \Psi+(K-\lambda I) \Psi=0 \\
\mathbf{n} \cdot \nabla \Psi=0 .
\end{array}
$$

This is an n-component spectral problem and the eigenfunctions are difficult to obtain in general, but if $u^{s}$ is a constant the eigenfunctions can be written $\Psi_{j n}=y_{j n} \phi_{n}$, where $\phi_{n}$ is a solution of the scalar eigenvalue problem

$$
\begin{aligned}
& \nabla^{2} \phi_{n}=-\alpha_{n}^{2} \phi_{n} \\
& \mathbf{n} \cdot \nabla \phi_{n}=0 .
\end{aligned}
$$

It follows that the vector $y_{j n} \in \Re^{m}$ is a solution of the algebraic eigenvalue problem

$$
\left(K-\mu_{n} \mathcal{D}-\lambda I\right) y=0,
$$

where $\mu_{n} \equiv \alpha_{n}^{2} \nu$. Generically the eigenfunctions are complete, and the solution of the variational equation can be written

$$
\xi(\zeta, \tau)=\sum_{n=0}^{\infty} e^{\left(K-\mu_{n} \mathcal{D}\right) \tau} y_{n} \phi_{n}(\zeta),
$$

where the $y_{n} \in \Re^{m}$ are determined by the initial data ${ }^{\dagger}$. Thus stability in an $L^{2}(\Omega)$ sense for the linearized reaction-diffusion equation with homogeneous boundary conditions is governed by the eigenvalues of the family $\left\{K-\mu_{n} \mathcal{D}\right\}_{n=0}^{\infty}$. It is also well-known that the principle of linearized stability holds for equations like (2.5), which means that asymptotic stability or instability for the zero solution of the variational equations implies the same properties of the solution $u^{s}$ of the nonlinear equations.

The spatial eigenfunction $\phi_{n}(\zeta)$ determines the spatial variation of that mode, but this has the equivalent form

$$
\phi_{n}(\zeta)=\phi\left(\alpha_{n} \zeta\right)=\phi\left(\frac{\alpha_{n}}{L} r\right)
$$

where $\phi$ is the solution of

$$
\nabla^{2} \phi=-\phi \text { in } \Omega
$$

with homogeneous Neumann boundary conditions. Thus all of the spatial modes can be obtained from a universal function by appropriately scaling the argument, but this only holds in the case of homogeneous boundary conditions.

Since the Laplacian has a compact inverse for a reasonable domain, the variational problem has a discrete spectrum, but it is convenient at present to replace $\mu_{n}$ with a continuous variable. Then stability of $u^{s}$ is governed by the character of the eigenvalues of the one-parameter family of matrices $\{K-\mu \mathcal{D}\}$ for $\mu \in \Re^{+}$, and a diffusive instability is defined as follows [56].

\footnotetext{
${ }^{\dagger}$ The mathematical structure of the solution is essentially unchanged if one considers a regular lattice of cells that interact with their neighbors via a passive diffusive flux proportional to the concentration differences; all that changes is that the sum is finite $[169,7]$.
} 
Definition 1. Suppose that $u^{s}$ is asymptotically stable as a steady-state solution of $u^{\prime}=R(u, p)$. We say that a zero-amplitude diffusive instability of $u^{s}$ exists if there exist $\mu^{ \pm}, 0<\mu^{-} \leq \mu^{+}<\infty$, such that $K-\mu \mathcal{D}$ has at least one eigenvalue with a positive real part when $\mu \in\left(\mu^{-}, \mu^{+}\right)$. If for some $\mu^{*} \in\left(\mu^{-}, \mu^{+}\right), K-\mu^{*} \mathcal{D}$ has a single real positive eigenvalue the instability is stationary at $\mu^{*}$, while if $K-\mu^{*} \mathcal{D}$ has complex eigenvalues with a positive real part the instability is oscillatory at $\mu^{*}$.

Thus whenever the first eigenvalue that crosses from the left-half plane (LHP) to the righthalf plane (RHP) is real the instability is stationary, and generically this leads to bifurcation of a stationary solution from $u^{s}$, whereas oscillatory instabilities generically lead to bifurcation of periodic solutions from $u^{s}$.

In the study of pattern formation one is interested in conditions on the kinetics under which Turing instabilities can arise, and the following theorem summarizes necessary conditions for stability of a uniform steady state. From this one can identify the type of kinetic interactions that can potentially give rise to diffusive instabilities under appropriate conditions on the parameters. In the theorem, $K\left[i_{1}, i_{2}, \cdots, i_{p}\right]$ denotes a $p \times p$ principal submatrix of $K$ formed from rows and columns $i_{1}, i_{2}, \cdots, i_{p}$ for $1 \leq p \leq n-1$.

Theorem 2..1. [163] Let $\mathcal{D}$ be diagonal with $\mathcal{D}_{j} \geq 0$. In order that $\sigma(K-\mu \mathcal{D}) \subset L H P$ for all such $\mathcal{D}$ and all $\mu \in[0, \infty)$, it is necessary that

- $\sigma(K) \subset L H P$

- $\sigma\left(K\left[i_{1}, i_{2}, \cdots, i_{p}\right]\right) \subset \overline{L H P}$ for all $p^{\text {th }}$-order submatrices of $K$, where $1 \leq p \leq n-1$.

These conditions are also sufficient for linearized stability for all $\mu \in[0, \infty)$ for $n=2$ and $n=3$.

The physical interpretation of how instabilities arise at positive wavelengths when one of the conditions in Theorem 2..1 is violated goes as follows. Suppose that there is an unstable subsystem of order $p$ represented by $K_{1}$, and suppose that $K$ is partitioned as in the proof of Theorem 2..1, i.e.,

$$
K=\left[\begin{array}{l|l}
K_{1} & K_{2} \\
\hline K_{3} & K_{4}
\end{array}\right]
$$

By hypothesis, the complementary subsystem, whose matrix is $K_{4}$ and which comprises species $p+1$ through $n$, is stable. The off-diagonal blocks $K_{2}$ and $K_{3}$ represent the interaction between the two subsystems. Were $K_{2}$ and/or $K_{3}$ identically zero, the two subsystems would be uncoupled and the composite system of species $1 \ldots n$ would be unstable. However, because the unstable subsystem is embedded in a larger network, the instability in the subsystem can be suppressed through stabilizing interactions with the rest of the network in a spatially-homogeneous system.

When spatial variations are allowed, the amplitudes of the Fourier components of any disturbance satisfy the equation

$$
\frac{d y_{k}}{d \tau}=\left(K-\mu_{k} \mathcal{D}\right) y_{k}
$$

and if we choose

$$
\mathcal{D}=\left[\begin{array}{l|l}
0 & 0 \\
\hline 0 & I
\end{array}\right]
$$


and partition $y_{k}$ appropriately, then

$$
\begin{aligned}
& \frac{d y_{k 1}}{d \tau}=K_{1} y_{k 1}+K_{2} y_{k 2} \\
& \frac{d y_{k 2}}{d \tau}=K_{3} y_{k 1}+\left(K_{4}-\mu_{k} I\right) y_{k 2} .
\end{aligned}
$$

Since $\sigma\left(K_{1}\right) \cap R H P \neq \emptyset, y_{k 1}(\tau)$ grows exponentially if $y_{k 2}(\tau) \equiv 0$. Of course $y_{k 2}(\tau) \not \equiv 0$ initially, but if $\mu_{k}$ is large enough it will decay rapidly and its stabilizing effect on $y_{k 1}$ will be lost. Thus diffusion offsets the influence of the stable subsystem by quenching the amplitude of the stable components.

A similar analysis can be done when the reference solution is a spatially-uniform periodic oscillation, rather than a steady state, and the result is that synchronized oscillations can also be destabilized when the diffusion coefficients differ appropriately [162].

\subsubsection{Diffusive instabilities for two component systems}

When a diffusive instability cannot be ruled out, a more detailed analysis of how the intervals of unstable wave numbers depend on the linearized kinetic parameters and on the ratio of diffusion coefficients for a two-component system goes as follows ( $c f$. [161, 149]). When there are only two components the only diffusive instabilities possible are stationary for non-negative diffusion coefficients, and thus instability occurs if $\operatorname{det}(K-\mu \mathcal{D})$ becomes negative for some $\mu$. Suppose that the second component is self-activating, which means that $k_{22}>0$, then one finds that $\mu^{ \pm}>0$ only if $\theta \equiv D_{2} / D_{1}<-k_{22} / k_{11} \ddagger$. Furthermore, for fixed $k_{i j}$ the discriminant of $\operatorname{det}(K-\mu \mathcal{D})$ is a quadratic in $\theta$, the roots of which are

$$
\theta^{ \pm}=\frac{\operatorname{det} K-k_{12} k_{21} \pm 2 \sqrt{-k_{12} k_{21} \operatorname{det} K}}{k_{11}^{2}} .
$$

It can be shown that

$$
0<\theta^{-}<\frac{-k_{22}}{k_{11}}<\theta^{+}
$$

and therefore the maximum allowable $\theta$ for which a zero-amplitude diffusive instability occurs at some $\mu>0$ is $\theta^{c} \equiv \theta^{-}$. Note that if trace $K=\operatorname{det} K=0$, then $\theta^{ \pm} \rightarrow 1$, which means that a diffusive instability can occur when the ratio of the diffusion coefficients is arbitrarily close to one, provided that both eigenvalues of the Jacobian for the kinetics are sufficiently close to zero. This conclusion carries over to $n$-component systems as well [182].

At the critical value $\theta$ of the ratio of diffusion coefficients, the critical $\mu$ is

$$
\mu^{+}=\mu^{-} \equiv \mu^{c}=\frac{k_{22}+\theta^{c} k_{11}}{2 \theta^{c}}
$$

\footnotetext{
${ }^{\ddagger}$ In section 5.2.1. Figure 14, we display representative examples of the types of stationary patterns that can emerge for a specific two-component system in two space dimensions.
} 
and the corresponding critical wave number is

$$
\frac{\alpha^{c}}{L} \equiv \sqrt{\frac{\mu^{c} \omega}{D_{1}}}=\sqrt{\frac{\omega}{D_{1}}\left(\frac{k_{22}+\theta^{c} k_{11}}{2 \theta^{c}}\right)} .
$$

The reciprocal of this defines a natural or intrinsic wavelength at marginal instability, which Turing [219] called the chemical wavelength. It is determined by the physico-chemical properties of the system, in particular the kinetic sensitivities at the steady state and the diffusion coefficients, but is independent of any geometric properties of the domain. In a one-dimensional system of length $L$ with homogeneous Neumann boundary conditions, $\alpha_{n}=n \pi, n=0,1, \cdots$ and $\phi_{n}=\cos n \pi x / L$, while under homogeneous Dirichlet conditions $\alpha_{n}=n \pi, n=1, \cdots$ and $\phi_{n}=\sin n \pi x / L$. In either case the critical wave number is indexed by the integer $n$ closest to

$$
\frac{L}{\pi} \sqrt{\frac{\omega}{D_{1}}\left(\frac{k_{22}+\theta^{c} k_{11}}{2 \theta^{c}}\right)}
$$

(see [163]). If $K$ and $\mathcal{D}$ are such that there is a positive real eigenvalue of $K-\mu \mathcal{D}$ for $\mu \in\left[\mu^{-}, \mu^{+}\right]$, then perturbations containing the mode $\phi_{n}$ are unstable when

$$
L \in\left[L_{n}^{-}, L_{n}^{+}\right]=\left[n \pi \sqrt{\frac{D_{1}}{\omega \mu^{+}}}, n \pi \sqrt{\frac{D_{1}}{\omega \mu^{-}}}\right] .
$$

In a slowly growing system, one expects that as $L$ increases from zero the uniform steady state always loses stability with respect to $\phi_{1}$ first and is linearly unstable with respect to $\phi_{1}$ over the range

$$
\Delta L_{1} \equiv L_{1}^{+}-L_{1}^{-}=\pi \sqrt{\frac{D_{1}}{\omega}}\left[\frac{1}{\sqrt{\mu^{-}}}-\frac{1}{\sqrt{\mu^{+}}}\right] .
$$

A more detailed analysis of growth shows that this is correct for growth that is very slow compared to the reaction and diffusion dynamics, but a more detailed analysis is needed in general, as will be shown later.

Assuming that growth is sufficiently slow, a simple measure of the range for which the qualitative features of small-amplitude patterns are invariant under changes in length is the interval of instability for the $n^{\text {th }}$ mode, which is given by

$$
\Delta L_{n}=n \pi \sqrt{\frac{D_{1}}{\omega}}\left[\frac{1}{\sqrt{\mu^{-}}}-\frac{1}{\sqrt{\mu^{+}}}\right] .
$$

Whenever $n$ is large enough or $\theta$ is sufficiently small, the intervals overlap and there is more than one mode to which the uniform steady state is unstable. In this case it is possible that more than one spatially-varying solution is stable or that the spatial distribution of the morphogens in the stable solution bears little resemblance to the spatial variation of the eigenfunctions. Thus linear analysis gives little information about scale-invariance in the full nonlinear model in these cases, but it 
emphasizes again the fact that Turing's model does not show perfect scale-invariance. Models with different boundary conditions on different species, for instance homogeneous Neumann on some species and homogeneous Dirichlet conditions on the others, show greater insensitivity to changes in scale [56], and perfect scale-invariance can be achieved by modulating either the kinetic coefficients or the diffusion coefficients appropriately, as is discussed later.

\subsection{Other morphogen transport mechanisms}

In either Turing's model or models based on source-sink mechanisms, the system is regarded as a homogeneous medium in which reaction occurs and transport is by passive diffusion as described by Fick's law, for which the flux of a species is proportional to the spatial gradient of that species. However there are several other modes of cell-cell communication, including the following.

- Indirect transfer via secretion into the extracellular space followed by re-uptake by a variety of mechanisms. The analog of Turing's problem for this mode of communication has been analyzed for passive secretion and uptake mechanisms [163], but detailed models of endoand exocytosis have not been included in this context. This is a formidable task, as the example of the Drosophila wing disc shown in Figure 2 suggests.

- All-to-all communication, in which all cells release the signaling species and all detect and perhaps internalize the signal [164]. While not useful for establishing a spatial pattern, this mode does serve as a quorum-sensing mechanism $[16,8,78]$ that can control gene expression and cell motility, and may be involved in size-regulation in some systems [95].

- Direct cell-cell communication, either via gap junctions or direct receptor-ligand interactions (which is often called juxtacrine signaling). Numerous examples of both types are known: in the Drosophila melanogaster, wing disc cells are coupled via gap junctions [232], but juxtacrine signaling via Notch and various ligands is used for establishing compartment boundaries or directing neighboring cells to adopt different fates.

- Active transport, for instance along microtubules, may play a role in polarizing cells.

Several of these mechanisms play an important role in the patterning of the wing disc in Drosophila, as illustrated in Figure 2.

Frequently the complexity involved in the interactions of the different processes is hidden by analyzing the spread of morphogens using a simple reaction-diffusion system such as (2.3) [110], but how to relate the individual steps in what may be a very complex process to such a high-level description is not known.

Processes such as directed transport along microtubules require different descriptions of transport, and we discuss two of them here. The first is a generalization of the simplest Brownian motion model that leads to Fick's law, in which particles execute a random walk in space due to their interaction with the background particles in the medium. We call this the position- or spacejump process, since it comprises a sequence of alternating pauses and jumps. In pure molecular diffusion the pauses are negligible but when, for example, binding is allowed, the duration of a 


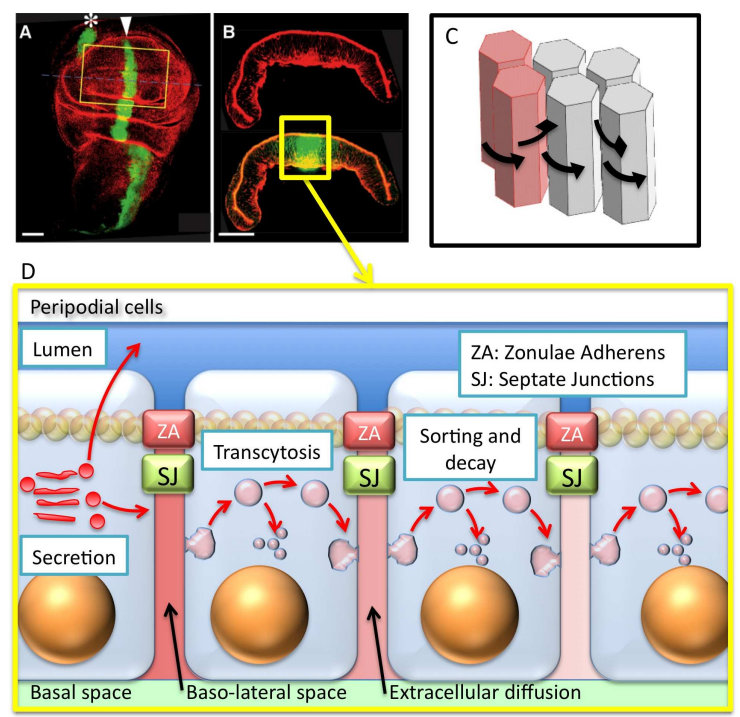

Figure 2: Patterning of epithelial cells in the Drosophila wing imaginal disc. The morphogen Dpp patterns the Anterior/Posterior compartments of the Drosophila wing imaginal disc in (A) top view showing the pouch and (B) slice (along dotted line in (A)) showing the geometry of the columnar cells. (C-D) Dpp establishes a non-uniform distribution to pattern the anterior/posterior axis by transport and reaction. Numerous processes may contribute to formation of the Dpp distribution including diffusion around columnar cells $(\mathrm{C})$ or transcytosis through columnar cells (D). Dpp secreted in the basolateral space cannot enter the lumen and vice versa due to the presence of septate junctions (SJ) in (D).

pause is governed by an exponential waiting time distribution. Furthermore, the direction of the steps is uniformly distributed and the distribution of lengths sharply peaked. A general formulation that can be reduced to the simplest diffusion process leads to the renewal equation

$$
P(\mathbf{x}, t \mid 0)=\hat{\Phi}(t) \delta(\mathbf{x})+\int_{0}^{t} \int_{R^{n}} \phi(t-\tau) T(\mathbf{x}, \mathbf{y}) P(\mathbf{y}, \tau \mid 0) d \mathbf{y} d \tau
$$

wherein $P(\mathbf{x}, t \mid 0)$ is the probability that a particle that begins at the origin at time 0 will be at $x \in$ $\Omega \subset \mathbf{R}^{n}$ at time $t, \phi$ is the waiting time distribution, $\hat{\Phi}$ is the complementary cumulative waiting time distribution, and $T$ is a kernel that determines the next position $\mathbf{x}$ for a walker currently at $\mathbf{y}$ $[165]^{\S}$. The primary assumption in this formulation is that the waiting time between jumps and the size and direction of a jump are uncoupled. Under suitable assumptions concerning the existence of limits as the mean step size goes to zero and the frequency of stepping goes to infinity, the process is governed by a diffusion equation, but other evolution equations will result for other waiting time distributions or other jump kernels. Using this formalism it is easy to incorporate 'resting' or transient 'trapping' states, and thereby to model various modes of binding or temporary localization. If for example, the waiting time distribution has a sufficiently fat tail this can lead to what is called 'sub-diffusion', for which the mean square displacement of a particle is characterized by $<r^{2}>\propto t^{\alpha}$, for $\alpha<1$ [194].

\footnotetext{
$\S$ This result is a generalization of an earlier result due to Scher and Lax [195] for lattices. The authors of [165] were unaware of the earlier result until very recently.
} 


\subsection{The mathematical description of chemotaxis}

Formation of spatial patterns of morphogens and cell determination may also involve cell movement in addition to reaction and transport. A dramatic example of this occurs in the formation of pigmentation patterns, which are discussed in Section 5. For instance in fish, various types of pigment cells migrate in the epithelium to form the variety of spots and stripes that are observed. Although it has not been established experimentally, it is likely that pigment cells respond to a spatial distribution of chemical attractants and repellents, and thereby establish the spatial pattern of pigment cells. A phenomenological model of cell movement in response to chemoattractants or chemorepellents leads to what is called the classical chemotaxis equation or the Patlak-Keller-Segel (PKS) chemotaxis equation (2.18), and this has been applied to model chemotactic movement in many different contexts $[104,105,106,180,70,128,4,192]$. In Sections 4. and 5. we summarize models of bacterial pattern formation in $E$. coli and fish pigmentation, respectively, that employ the PKS equation. Here we provide a brief introduction to the derivation of this equation.

Let $n(x, t)$ denote the cell density as a function of space and time, and suppose that in the absence of chemosignals cells execute an unbiased random walk. Under a suitable scaling of space and time, $n$ evolves according to a diffusion process governed by

$$
\frac{\partial n}{\partial t}=\nabla \cdot\left(D_{n} \nabla n\right)
$$

where $D_{n}$ is the diffusion coefficient of the cells. The diffusive flux is given by $j=-D_{n} \nabla n$, which is simply Fick's law applied to cells. In the presence of a gradient of a chemosignal one postulates that the random walk is biased, which gives rise to a chemotactic drift. To incorporate the drift, the phenomenological form

$$
j=-D_{n} \nabla n+\chi(n, S) n \nabla S
$$

is often used, and the evolution equation for cell density becomes

$$
\frac{\partial n}{\partial t}=\nabla \cdot\left(D_{n} \nabla n-\chi n \nabla S\right)
$$

The coefficient $\chi=\chi(n, S, \ldots)$ is called the chemotactic sensitivity, $u_{s}=\chi \nabla S$ is called the macroscopic chemotactic velocity, and equation (2.18) is called the classical chemotaxis or PKS equation.

Equation (2.18) was first derived by Patlak [181] from a random walk with 'persistence of direction' and 'external bias'. Persistence of direction means that the direction of the next step is correlated with that of the previous step, whereas external bias means there can be external forces on the particle or other external factors that bias the movement. Equation (2.18) results when the bias is caused by chemosignals. Later Keller and Segel [104] derived a system of four parabolic equations to model the chemotactic movement of Dictyostelium discoideum, and reduced it to (2.18) and the following reaction-diffusion equation for the chemosignal

$$
\frac{\partial S}{\partial t}=D_{s} \Delta_{s} S+R(n, S)
$$


They suggested that the aggregation of cells results from an instability of the uniform steady state, but it is now known that this is not the correct explanation; aggregation results from the response of cells to waves of a chemoattractant that are initiated by pacemaker cells. Later, similar equations were applied to bacterial chemotaxis, with the aim of explaining the traveling bands observed in $E$. coli by Adler $[1,105,106]$. Here too the conclusion is in doubt because the authors required that the chemotaxis sensitivity $\chi=\chi_{0} / S$ become singular at $S=0$ in order to establish the existence of a traveling wave.

More recently (2.18) and (2.19) have been studied extensively mathematically (see [92] for a detailed review and references). These analyses were motivated by biological features of chemotactic movement and the spatial patterns that result. Existence of stable non-uniform steady states and blow-up solutions were explored to determine if the model can produce patterns of cell concentration or aggregation [41, 98, 88, 170]. Existence of traveling wave solutions has been studied to determine whether the model can produce patterns corresponding to the traveling bands of bacteria observed experimentally $[106,103,93]$. Many variations on the minimal form given by $(2.18$, 2.19) have been developed to incorporate mechanisms, such as excluded volume, that prevent the blow-up solutions in finite time (see [90] for a review). Equations that describe taxis in the presence of multiple signals have also been derived under various cases [174, 240].

A major drawback in using the phenomenological approach that leads to (2.18) is how one rationalizes the constitutive assumption (2.17). Some characteristics of the microscopic response of individual cells have been incorporated into the chemotactic sensitivity $\chi$ in different contexts. Keller and Segel [105] related the chemotactic sensitivity to the frequency of reversals of a particle moving along the real line, and Segel [198] incorporated receptor dynamics. Ford and Lauffenburger $[72,71]$ have estimated taxis coefficients within the framework of the phenomenological approach. Pate and Othmer [180] derived the chemotactic velocity of amoeboid (crawling) cells in terms of forces exerted by the cells, beginning from Newton's law for the motion of a point particle, neglecting inertial effects, and assuming that the motive force exerted by a cell is a function of the attractant concentration. They showed how the chemotactic sensitivity is related to the rate of change of the force with attractant concentration, and in this approach the dependence of the flux on the gradient of the attractant arises from the difference in the force exerted in different directions due to different attractant concentrations. Experimental support for this approach comes from the work of Varnum-Finney, Voss, and Soll [226], who show that in Dictyostelium discoideum, as many pseudopods are produced down-gradient as up-gradient, but those that are up-gradient are more successful in generating cell movement. Another approach, based on a continuous time reinforced random walk in which the walker modifies the transition probabilities of an interval for successive crossings, is developed in [170] for a single tactic substance and in [174] for multiple substances.

Although the PKS equation has been used widely in modeling chemotactic movement and other kinds of tactic movement, the general problem of systematically incorporating microscopic details of the cell response into the chemotactic sensitivity remains a challenge. However, some progress has been made on incorporating characteristics of cell-level behavior into the classical description for the movement of bacteria and Dictyostelium discoideum [89, 166, 64, 63, 65, 240]. In Section 4.4. we discuss this aspect in the context of bacterial chemotaxis. The starting point for these 
analyses is a second major type of movement called a velocity-jump process. In this process the motion consists of a sequence of "runs" separated by reorientations, during which a new velocity is chosen. This leads to the following evolution equation for the density $p(\mathbf{x}, \mathbf{v}, t)$ of individuals at a spatial position $\mathbf{x} \in \Omega \subset \mathbf{R}^{n}$, that are moving with velocity $\mathbf{v} \in V \subset \mathbf{R}^{n}$ at time $t \geq 0$ [165]:

$$
\frac{\partial}{\partial t} p(\mathbf{x}, \mathbf{v}, t)+\mathbf{v} \cdot \nabla p(\mathbf{x}, \mathbf{v}, t)=-\lambda p(\mathbf{x}, \mathbf{v}, t)+\lambda \int_{V} T\left(\mathbf{v}^{\prime}, \mathbf{v}\right) p\left(\mathbf{x}, \mathbf{v}^{\prime}, t\right) d \mathbf{v}^{\prime} .
$$

The parameter $\lambda$ is the turning rate ( $1 / \lambda$ is the mean run time between jumps), and in general it may be space-dependent and depend on internal and external variables as well. The turning kernel $T\left(\mathbf{v}, \mathbf{v}^{\prime}\right)$ defines the probability of a jump from $\mathbf{v}^{\prime}$ to $\mathbf{v}$ when a jump occurs, and implicit in this formulation is the assumption that the choice of a new velocity is independent of the run length. The turning kernel may also be space-dependent, and it may depend on the total density of individuals. As will be shown in Section 4., when this approach is applied to the bacterium E.coli the turning frequency must depend on the extracellular signal, as transduced through the signal transduction and motor control system. The situation is more complex when this approach is applied to amoeboid cells such as Dictyostelium discoideum, which use both run length control and taxis [69], because there the extracellular cAMP distribution affects the forces involved in the turning process and these must be accounted for explicitly [65]. As in the space-jump process, resting states can be incorporated, as can birth-death processes [89]. It is also straightforward to develop a model of intracellular transport that uses both kinesin and dynein (which move in opposite directions along microtubules) using the velocity-jump process.

\subsection{The problem of robustness and scale-invariance}

The entire process of development, including growth and pattern formation, can be characterized by its tendency to produce a normal adult, or to regulate, in the face of various disturbances imposed during development. At one extreme is mosaic development in which removal of a part of a developing embryo at one stage results in the absence of that part (or parts that develop from it) in later stages. In mosaic eggs such as annelids and mollusks, removal of portions of the cytoplasm from the egg leads to the absence of those organs that normally develop from the excised region. On the other hand, many systems show a high degree of regulation, particularly at early stages of development. For instance, each of the cells that results from the first cleavage in amphibian eggs can, when separated from the other, develop into a normal, although usually smaller, adult. Regeneration of lost limbs in Hydra, a small marine creature, is an example of regulation in a system in its terminal state of development. Development is rarely either strictly mosaic or strictly regulative at all stages, but rather, shows both characteristics at different stages. Certainly regulation requires some form of intercellular communication and some kind of feedback mechanism whereby removal of part of an organism is sensed by the remainder and development redirected to compensate for the part removed. The current thinking is that such a mechanism either coincides with or is a part of the mechanism that controls pattern formation.

To understand what we mean by robustness of a model, consider the dynamical system

$$
\frac{d x}{d t}=F(x, p, S(t)) \quad x(0)=x_{0}
$$


wherein $p$ is a vector of model parameters and $S(t)$ is a time-dependent input. Three classes of 'perturbations' lead to three types of 'insensitivity' or 'robustness'.

1. Insensitivity with respect to inputs, by which we mean that some components of the state of the system 'ignore' a certain class of deterministic inputs in the long run. This type of robustness is a characteristic of systems in which one or more components adapt to constant signals, such as the motor control protein Chey $p$ in E. coli.

2. Insensitivity with respect to changes in the vector field, i.e., the function $F$ in (2.21). This is captured in the notion of coarseness or structural stability: a vector field is structurally stable if its associated flow is orbit equivalent to the flow generated by any vector field in a sufficiently small neighborhood in a suitable topology. This is the broadest definition, for it includes changes in $F$ itself, but it is a strictly local (in a suitable topology) result, except for linear vector fields.

3. Insensitivity with respect to changes in parameters. Strictly speaking this is a subset of (2), but is often considered by itself.

4. Closely related to all of these is insensitivity to either internally-generated noise, due for example, to molecular fluctuations, unequal partition of molecules at cell division, etc, or to external noise due to fluctuations in the system environment. The main difference between this case and the first three is that noise is usually thought to be persistent in time and is often small amplitude, whereas, E. coli, for instance, can adapt to signal amplitudes ranging over five orders of magnitude.

A first step is to decide what characteristic of the dynamics is being tested for robustness. In the context of spatial pattern formation, it is often a measure of the size of the system that is of interest with respect to robustness. As we have seen, reaction-diffusion systems, either with or without specialized source regions, are often used to describe pattern formation, and as we saw in the simple case of the French flag and in the analysis of Turing systems, the standard models do not produce scale-invariant patterns. In either case the spatial distribution of a morphogen is governed by the intrinsic kinetic and diffusion time scales, and thus scale-invariance may be achieved by suitably modulating either one in a size-dependent manner. One way of doing this is to modulate these scales with a species produced by all cells. The essential idea can be illustrated in a system having only one morphogen.

Let $c$ denote the concentration of the morphogen and let $m$ denote the concentration of the modulating or control species. Suppose that they react and diffuse in the region $\Omega$ according to the following equations

$$
\begin{aligned}
\frac{\partial c}{\partial t} & =\nabla \cdot(D(m) \nabla c)+\lambda(m) R(c) & & \text { in } \Omega \\
\frac{\partial m}{\partial t} & =D_{m} \nabla^{2} C+R & & \text { in } \Omega \\
-n \cdot(D(\cdot) \nabla c) & =0 & & \text { on } \partial \Omega \\
m & =0 & & \text { on } \partial \Omega .
\end{aligned}
$$


As indicated, either or both of the diffusion coefficient or the characteristic kinetic time scale of the morphogen can be modulated by the control species [167]. In effect, the former changes the underlying space metric by changing the diffusion coefficient, and the latter changes the time scale by modulating the reaction rates. If only diffusion is modulated then the diffusion coefficients must scale as $L^{2}$, which in effect changes the metric, since the level sets of the control species provide a measure of distance from the boundary. In Drosophila melanogaster and related dipterans, it is unlikely that the diffusion coefficients are modulated [81], but it is shown in Umulis et al [223] that if the total number of nuclei does not change with the size of the embryo and other conditions are met, then the morphogen gradient will scale with size, as is observed experimentally [81]. This invariance can be understood by thinking of the total number of nuclei as embedded in a rubber sheet, and then stretching the sheet to fit the embryo size. Thus the density of nuclei scales as $L^{-2}$, which is what is required to produce the correct scaling of the morphogen distribution.

\subsection{The role of growth and cell movement in pattern formation}

In some systems growth, cell movement and patterning occur on comparable time scales and there may be feedback between them. An example treated later arises in pigmentation patterns in fish. Here we consider the following model for growth and cell movement in a two-dimensional layer of cells (for details see Section 5. and Painter, et al., [173]). Assume that (i) there are two cell types, which may have different diffusion constants and chemotactic sensitivities, and (ii) there are $n$ morphogens which diffuse and react throughout the system. We suppose that morphogens make a negligible contribution to the total mass and momentum of the tissue.

Let $\rho_{i}$ be the density in mass/volume of the $i^{t h}$ cell type and suppose that the total density is constant. Then the overall continuity equation for the cell mass reads

$$
\frac{\partial \rho}{\partial t}+\nabla \cdot(\rho v)=\sum \rho_{i} \mathcal{R}_{i}
$$

where $\mathcal{R}_{i}$ is the net rate of creation or conversion of the $i^{\text {th }}$ cell type per unit of mass of that type. Since $\rho$ is constant by hypothesis we have

$$
\rho \nabla \cdot v=\sum_{i} \rho_{i} \mathcal{R}_{i}
$$

or

$$
\nabla \cdot v=\sum_{i} \omega_{i} \mathcal{R}_{i}
$$

where $\omega_{i}$ is the mass fraction of the $i^{\text {th }}$ species. Note that there are no diffusion terms in (2.22) because we define the velocity $v$ via

$$
v=\sum \omega_{i} v_{i}
$$

The balance equations for the individual cell types are

$$
\frac{\partial \rho_{i}}{\partial t}+\nabla \cdot\left(\rho_{i} v_{i}\right)=\rho_{i} \mathcal{R}_{i}
$$


and we rewrite this as

$$
\begin{aligned}
\frac{\partial \rho_{i}}{\partial t}+\nabla \cdot\left(\rho_{i} v\right) & =-\nabla \cdot \rho_{i}\left(v_{i}-v\right)+\rho_{i} \mathcal{R}_{i} \\
& =-\nabla \cdot j_{i}+\rho_{i} \mathcal{R}_{i}
\end{aligned}
$$

where $j_{i}$ is the flux relative to the mass-average velocity. This is comprised of diffusive and tactic components. Clearly if we sum (2.25) over $i$ and use the definition of $\rho$ and $v$ we obtain (2.22) and hence (2.23). We define the fluxes $j_{i}$ as

$$
j_{i}=\rho_{i}\left(v_{i}-v\right)=-D_{i} \nabla \rho_{i}+\chi_{i}(u) \rho_{i} \nabla u_{i},
$$

where $u_{i}$ is the attractant or repellent for the $i^{t h}$ cell type, and $\chi_{i}$ is the chemotactic sensitivity of that type to $u_{i}$. Some or all of the sensitivities may be zero, and cells may be sensitive to multiple signals. If we sum (2.28) over $i$ we obtain

$$
\sum_{i} j_{i}=0
$$

Thus we have the desired 'counter-transport' wherein one species displaces the other but the local density remains fixed.

We write a similar set of equations for the morphogens, amongst which we include the chemoattractants and repellents, and we assume that the morphogens are transported only by diffusion and convection, the former relative to the mass average velocity of the cells. Thus we write

$$
\frac{\partial u_{i}}{\partial t}+\nabla \cdot\left(u_{i} v\right)=u_{i} R_{i}
$$

where $u_{i}$ is the mass density of the $i^{t h}$ morphogen, which is used because $v$ represents the mass average velocity. Both the cell density and morphogen densities could be expressed in molar terms as well, and then the molar average velocity would appear in the equations.

We must now postulate the velocity field to avoid solving the momentum equations as well. Since

$$
\nabla \cdot v=\sum \omega_{i} \mathcal{R}_{i}
$$

the simplest hypothesis is that the cell growth rates $\mathcal{R}_{i}$ are all identical, and then in two space dimensions

$$
\nabla \cdot v=\frac{\partial v_{x}}{\partial x}+\frac{\partial v_{y}}{\partial y}=R
$$

This can be satisfied by postulating that

$$
V=\frac{R}{2}(x \mathbf{i}+y \mathbf{j}) .
$$

This implicitly assumes a coordinate frame fixed at one corner of the tissue, which grows at equal rates per unit length in each direction. With this assumption (2.26) becomes

$$
\frac{\partial \rho_{i}}{\partial t}+\rho_{i} \nabla \cdot v+v \dot{\nabla} \rho_{i}=-\nabla \cdot j_{i}+\rho_{i} R
$$


or

$$
\frac{\partial \rho_{i}}{\partial t}+\frac{R}{2} x \frac{\partial \rho_{i}}{\partial x}+\frac{R}{2} y \frac{\partial \rho_{i}}{\partial y}=-\nabla \cdot j_{i} .
$$

Note that the growth rate has disappeared from this equation, because the 'convective flux' due to volume expansion on the left-hand side exactly balances the growth term on the right-hand side. If we now introduce new coordinates so as to map the growing domain onto the unit square via the transformation

$$
\xi=\frac{x}{L_{1}(t)}, \quad \nu=\frac{y}{L_{2}(t)}, \quad \tau=t
$$

then we find that

$$
\frac{\partial \rho_{i}}{\partial \tau}=\frac{1}{L_{1}^{2}} \frac{\partial}{\partial \xi}\left(D_{i} \frac{\partial \rho_{i}}{\partial \xi}-\chi_{i} \rho_{i} \frac{\partial u}{\partial \xi}\right)+\frac{1}{L_{2}^{2}} \frac{\partial}{\partial \nu}\left(D_{i} \frac{\partial \rho_{i}}{\partial \nu}-\chi_{i} \rho_{i} \frac{\partial u}{\partial \nu}\right) .
$$

Thus we see that in the moving frame the connective terms all disappear. Similar reductions hold for the morphogen equations. The convenience of such simplifications will be exploited in Section 5.4. to investigate pigmentation pattern control on the growing skins of fish. A more general analysis of patterning in growing domains appears in Crampin, et al., [49].

\section{Morphogen-mediated patterning in Drosophila melanogaster}

\subsection{Background}

While morphogen-mediated patterning has been studied in zebrafish [244], frogs [21], and other model organisms, the most extensive biological and mathematical work has been done on the model organism Drosophila melanogaster (the common fruitfly). During the first four hours of Drosophila development, morphogens pattern the anterior/posterior (AP) and dorsal/ventral (DV) axes into regions of differential gene expression that presage the relative spatial position of adult structures. Examples of the AP and DV protein distributions that result from zygotic gene expression are shown in Figure 3.

The developing embryo of Drosophila approximates a prolate ellipsoid $500 \mu \mathrm{m}$ long and $160 \mu \mathrm{m}$ in diameter at the midpoint that is surrounded by a $0.5 \mu \mathrm{m}$ thick fluid-filled shell called the perivitelline (PV) space. The AP axis extends from the anterior extremum, defined by the maternal localization of bicoid mRNA (discussed later) in early development, to the opposite extremum where nanos mRNA is maternally-localized. The DV axis, which lies perpendicular to the AP axis, is established early in development through activation of the transcription factor Dorsal in the ventral most region of the embryo $₫$.

Drosophila development is temporally divided into a number of distinct stages. Pattern formation begins during oogenesis, which occurs prior to fertilization and during which the mature

\footnotetext{
`Often Drosophila genes are named for their mutant phenotype rather than for the function of the gene product thus, for example, knocking out the transcription factor Dorsal results in an embryo forming only dorsal tissues.
} 


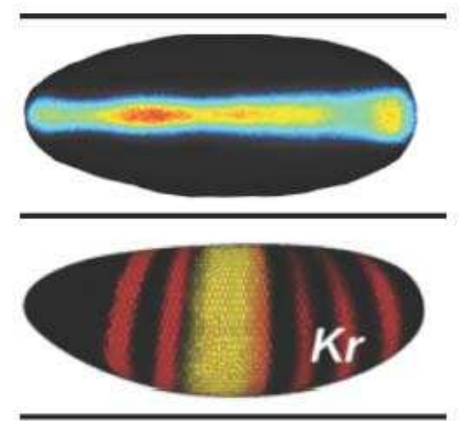

Figure 3: Morphogens pattern the anterior/posterior (AP) and dorsal/ventral (DV) axes during the first 4 hours of embryonic development. (A) The top view shows a population mean distribution of pMad signaling, a signaling intermediate for a step of the DV patterning network. (B) The side view shows the distribution of even-skipped and Kruppel (from [73] Cell with permission). (C) Cross sectional view showing nuclear and yolk/cytoplasm levels of the anterior morphogen Bicoid (from [83] with permission).

egg forms in the female fly egg chambers located in the ovaries. At $25^{\circ} \mathrm{C}$ oogenesis proceeds for approximately three days, through a distinct series of developmental stages. The separate developmental events are divided into 17 stages defined by the changes that occur in the morphological structure of the egg chamber [183].

Embryogenesis begins immediately after fertilization, and this process is divided into 17 stages based on distinct morphological changes in the developing embryo. Furthermore, due to the importance of early development, the first five stages are divided by cell-cycle number based on the sequence of synchronous nuclear divisions, beginning at fertilization. A very good approximation to the total number of nuclei in the syncytium at any given stage is $2^{n-1}$ where $\mathrm{n}$ is the current cycle (i.e. during the 14 th nuclear division cycle there are $\approx 2^{13}$ nuclei in the embryo). During the 14th mitotic division membranes begin to form between the nuclei that line the periphery of the embryo, and later they invaginate to delineate individual cells. Thus, during nuclear division cycle 14 there is an ambiguity as to whether nuclei or cells are lining the PV membrane. To further complicate matters, nuclei during this stage of development respond to local receptors, are surrounded by many cellular organelles, and secrete proteins in a spatially restricted manner similar to cells that participate in cell to cell signaling.

\subsection{Bicoid gradient formation and AP patterning}

Three genes - gurken, torpedo and cornichon, are currently known to be the primary factors for the initial formation of both the AP and DV axes [154, 183]. gurken gene expression originates in the germline cell whereas torpedo expression takes place in the somatic follicle cells. Absence of either gurken or torpedo produces eggs that lack polarity along both the AP and DV axes. Gurken, a signaling molecule, is transported with the aid of Cornichon from the endoplasmic reticulum to the oocyte surface where it is released through the membrane. Once released, Gurken is thought to interact with Torpedo, the Drosophila homologue of the Epidermal Growth Factor (EGF) receptor [154]. This early signal is believed to cause the terminal follicle cells to adopt a posterior fate 
and thus the origin of AP polarity [183]. This group of follicle cells returns a signal to the oocyte causing a reorganization of the cytoskeleton and reversed polarity of the microtubules. Microtubule polarity reversal allows transport of maternal RNAs bicoid and oskar to the anterior and posterior poles respectively via kinesin motors. The localized RNA remains until early embryogenesis where it becomes translated to further specify the AP axis.

bicoid mRNA tethered at the anterior pole encodes a transcription factor Bicoid (Bcd), which is a morphogen that specifies regions of gene expression in a concentration dependent manner [59]. The spatial distribution of Bicoid protein has a maximum near the anterior pole, where bicoid mRNA is produced, and decays monotonically to its minimum near the posterior pole [59] (see Figure 4). Bicoid promotes the transcription of the zygotic gene hunchback, which is expressed in the anterior half of the embryo. Hunchback acts as both a repressor and activator that specifies regions of expression for other targets called the gap genes [96]. A complex system of activating and repressing interactions ultimately generates the segmented body plan for Drosophila.

Analysis of Bicoid-mediated embryonic patterning has focused on two major questions: 1) what processes interact to regulate the dynamic, spatial distribution of the Bicoid protein, and 2) what processes contribute to the precise position and reproducibility of Bicoid target gene expression?

Early studies demonstrated that increases or decreases in maternally-supplied $b c d$ mRNA lead to increases or decreases in Bicoid levels in the embryo and subsequent shifts in the position of anterior structures [59]. The stained protein distribution of Bicoid is well approximated by a decaying exponential $[81,94]$, which arises automatically from a mathematical model for the diffusion of a molecule that undergoes first-order decay (see Section 2.1.). Since the protein distribution is approximated well by such a model it was widely assumed that the Bicoid distribution is established by simple diffusion and decay. The only remaining problem was to measure the rates of diffusion and linear decay.

To estimate the diffusion coefficient of Bicoid protein, investigators injected small amounts of dextran molecules of approximately the same molecular weight and size of Bicoid protein into Drosophila embryos [81]. The dynamic transport of the dextran molecules was imaged in live embryos and their diffusion constant estimated by fitting the image data to a mathematical model. The calculated diffusion coefficient of $\approx 20 \mu \mathrm{m}^{2} / \mathrm{sec}$ suggested that diffusion was rapid enough to be the principle means of Bicoid transport along the AP axis [81]. However, more recently it has been demonstrated that the in vivo diffusion rate for a Bicoid-eGFP (eGFP: enhanced Green Fluorescent Protein) fusion protein is much lower than the biologically inert dextran molecules [83].

In embryos stained for Bicoid protein, the majority of Bicoid accumulates in the nuclei lining the periphery of the embryo (See Figures $3 \mathrm{C}$ and $5 \mathrm{~A}$ ) and not in the core yolk cytoplasm. Since Bicoid concentrations are clearly higher in the nuclei, a natural hypothesis is that the nuclei play an important role in determining the dynamics and distribution of Bicoid [81, 46, 83]. To investigate the role of the nuclei, Gregor et al. [83] employed Fluorescence Recovery After Photobleaching (FRAP) to measure the import and export rates responsible for the exchange of Bicoid between the cytoplasm and the nuclei. The FRAP data were fitted to a linear local model for the number of 


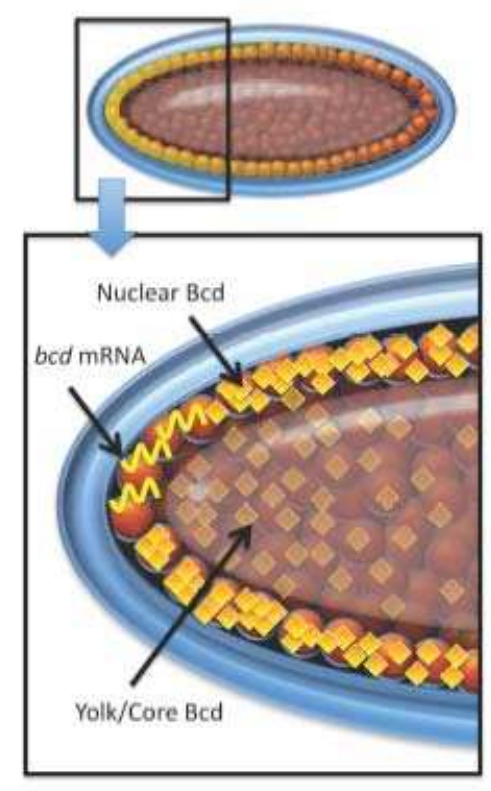

Figure 4: After fertilization, maternally-supplied bcd mRNA tethered at the anterior pole is translated to bicoid protein. The nonuniform spatial distribution of the protein has a maximum near the anterior end and decays posteriorly. There are numerous possible spatial pathways for Bicoid transport.

fluorescent Bicoid molecules $(n)$ in a single nucleus given by

$$
\frac{d n(t)}{d t}=k_{\text {in }} C_{\text {out }}-\frac{1}{\tau_{n}} n(t) .
$$

Here $k_{\text {in }}\left(k_{\text {out }}\right)$ is the import (export) rate, $k_{d-n u c}$ is the decay rate, $C_{\text {out }}$ is the concentration of Bicoid-eGFP, and $\tau_{n} \equiv\left(k_{\text {out }}+k_{d-n u c}\right)^{-1}$ is the time constant defined by the export and decay rates [83]. The solution of (3.1) for a reduction in the number of fluorescent molecules $\Delta n_{0}$ is

$$
\begin{aligned}
n(t) & =n_{\infty}-\Delta n_{0} \exp \left(-t / \tau_{n}\right), \\
n_{\infty} & =k_{\text {in }} \tau_{n} C_{\text {out }},
\end{aligned}
$$

and thus the recovery after photobleaching depends on the time-scale for nuclear export. The simple nuclear import-export and decay model was fitted to FRAP data, giving a calculated timeconstant of $\tau_{n} \approx 70 \mathrm{~s}$, which is sufficient for establishing a steady-state Bicoid distribution when coupled with the rapid diffusion approximated from the dextran molecules. Given the very rapid recovery after photobleaching, one would expect the concentration of Bicoid within each nucleus to remain constant or, if not constant, that the total number of Bicoid molecules in the nucleus would remain constant. During the course of the experiment, a further observation suggested another intriguing aspect of Bicoid-mediated patterning: as the nuclei grow, the concentration of Bicoid in each nucleus decreases at a rate inconsistent with either a fixed concentration or a fixed total number of molecules in the system. Gregor et al. found that the observed nuclear Bicoid 
concentration is predicted best by a model in which nuclei act as perfect absorbers, and transport to the nuclei is diffusion-limited. This assumption leads to the rate $k_{i n}=4 \pi r_{n} D$, where $r_{n}$ is the nuclei radius and $D$ is the diffusion constant, and with this, the diffusion constant of Bicoid in the cortical cytoplasm is predicted to be $\approx 0.37 \mu \mathrm{m}^{2} / \mathrm{sec}$, almost 100 -fold lower than the diffusion of dextran molecules. This yields a diffusion time $\tau_{D}=L^{2} / D$ of $\approx 150$ hours for the entire embryo length (or $\approx 9$ hours for one decay-length), far greater than the 3.5 hour development time. To directly measure the diffusion coefficient in vivo, Gregor et al. used FRAP in the cortical cytoplasm and calculated a diffusion coefficient of $\approx 0.3 \mu \mathrm{m}^{2} / \mathrm{sec}$, consistent with the predicted value.

It therefore appears that diffusion is too slow in the context of AP patterning in Drosophila, but a number of both experimental and modeling issues require resolution. First, in the model for nuclear transport of Bicoid, the extracellular concentration is assumed constant and it appears that the cytoplasmic content in the vicinity of the nuclei also remains constant. However, the photobleached Bicoid molecules exported from the nuclei will compete with fluorescent Bicoid for import. Since $D=\left(C_{\text {in }} / C_{\text {out }}\right)\left(r_{n}^{2} / 3 \tau_{n}\right)$, a small decrease in $\tau_{n}$ will lead to an increase in the predicted diffusion coefficient [83]. Additionally, the in vivo measurements for the diffusion constant $D$ were carried out in the cytoplasm near the embryonic periphery and technology does not permit direct measurement deeper in the embryo. The experiments only measure the concentration of Bicoid in the nuclei and cytoplasm of the embryo, not the flux of molecules in the different internal regions of the embryo. It is possible that the bulk of Bicoid transport occurs through regions of the embryo removed from the nuclei at the periphery, but once at the periphery the high import rates lead to nuclear accumulation of Bicoid.

Another intriguing possibility that was recently investigated is that the non-uniform distribution of Bcd protein depends on the non-uniform distribution of maternal $b c d$ mRNA. This suggests that the positional information may arise from processes that regulate the transcript transport in addition to the protein transport and additional work is needed to investigate the relationship between changes in the $b c d$ mRNA distribution and the resulting Bcd protein distribution [211].

To investigate alternative possibilities of Bcd patterning, the current generation of mathematical models will have to be extended to include a more realistic 3D geometry that better reflects the structure and allows a more accurate description of the underlying processes. For instance, a 3D FRAP model that accounts for both intra- and extra-nuclear bleached and fluorescent Bicoid molecules would provide additional insight into the transport and diffusion rates. In addition, more sophisticated 3D organism-scale models of the embryo would allow one to test alternative transport mechanisms and quantitatively determine the relative contributions of cortical vs. core Bicoid fluxes. These mathematical models will provide deeper insights and lead to additional biological experiments that quantitatively determine the mechanisms of Bicoid transport.

\subsection{Terminal gradient formation in Drosophila embryos}

Nuclei are also involved in patterning the terminal regions of the Drosophila embryo. The anteriorand posterior-most structures rely on a non-uniform spatial distribution of phosphorylated ERK/ MAPK [47] (ERK: extracellular-signal regulated kinase; MAPK: mitogen-activated protein kinase; 
the phosphorylated ERK is dpERK) in which dpERK is highest at the anterior and posterior poles, decaying to a minimum near the AP midline of the embryo (Figure $5 \mathrm{~B}$ ). The spatial distribution of intracellular dpERK relies on the spatial activation of Torso, a receptor tyrosine kinase (RTK), by ligands in the extracellular space surrounding the embryo [38, 47].

Two possible mechanisms could establish the spatially non-uniform dpERK profile: 1) extracellular regulation of the ligand and Torso receptor distribution similar to the extracellular regulation of BMPs (discussed later herein); or 2) diffusion and reaction of intracellular dpERK with nuclei and other intracellular components. To discern between these mechanisms, Coppey et al. [47] coupled mathematical models for nuclear import/export and long range diffusion with experiments to visualize the dpERK distribution in $w t$ and mutant embryos.

Consider the following morphogen patterning system: 1) morphogen is secreted at a localized source at one end of a field of binding sites (receptor or nuclei); 2) the morphogen is free to diffuse; and 3) the morphogen binds to receptors or is imported into nuclei, which removes them from the freely diffusing pool. Under the assumption of excess binding this leads to the following linear system

$$
\begin{aligned}
\frac{\partial c}{\partial t} & =D \frac{\partial^{2} c}{\partial x^{2}}-k_{o n} R_{t o t} c \\
D \frac{\partial c}{\partial x} & =J \text { at } x=0, \\
D \frac{\partial c}{\partial x} & =0 \text { at } x=L,
\end{aligned}
$$

where $c$ is the morphogen concentration, $D$ is the diffusion coefficient, $k_{o n}$ is the binding rate, and $R_{t o t}$ is the total concentration of binding sites. The above system is simply the time-dependent analogue of equations (2.1) in Section 2.1.. If the system length is much greater than the morphogen decay length, the equilibrium solution is therefore approximated by

$$
c(x)=\Lambda \exp \left(-x \sqrt{k_{o n} R_{t o t} / D}\right),
$$

and $\Lambda$ is determined by a balance of the input flux and model parameters. Given equation 3.5, it is readily apparent that increasing the binding site density (Torso or nuclei) leads to a morphogen distribution with a reduced range.

To determine whether intra- or extracellular events regulated the spatial distribution of dpERK, Coppey et al. increased and decreased Torso levels using genetic approaches. Changes in the Torso level yielded dpERK profiles that were indistinguishable from $w t$ dpERK staining patterns, a result only explainable in the context of the trapping model if the $w t$ embryos have excess Torso (high $R_{t o t}$ ), which greatly limits the range of the extracellular ligand. This indirect evidence led to the suggestion that the dpERK distribution may be mediated by intracellular rather than extracellular processes [47].

To investigate the intracellular processes regulating the dpERK pattern, it was hypothesized that the balance between nuclei import and export regulates the distribution of dpERK [47]. If nuclei shape the dpERK distributions, equation (3.5) suggests that increasing or decreasing the 
nuclei density would reduce or expand the range of dpERK signaling respectively. To investigate this dependence, Coppey et al. [47] imaged the distributions of dpERK in nuclear division cycle $n=10-14$ embryos (recall there are $\approx 2^{n-1}$ nuclei per cycle) and in mutants that locally disrupt the nuclear density. With this approach, three observations were made: 1) dpERK concentration in nuclei is between 1.4 and 2 times higher than in the surrounding cytoplasm; 2) the steepness of the dpERK distribution increases as the number of nuclei increases; and 3) the dpERK distribution is sensitive to changes in nuclear density as observed in shakleton mutant embryos. The combination of dpERK profile sharpening during the synchronous nuclear division cycles and the disruptions of dpERK in shakleton embryos demonstrates a clear relationship between nuclear density and morphogen profile [47].

One obvious extension of the Torso/dpERK model is to investigate the scale-invariance of dpERK distributions between different Drosophila species. Different species of Drosophila lay embryos varying greatly in size, but containing similar numbers of nuclei produced by synchronous nuclear division. Two groups have independently suggested that nuclei may play an important role in the scale-invariance of morphogen gradients such as Bicoid [223, 83]. The main hypothesis central to this theory is that if nuclei bind the morphogen that is otherwise free to diffuse, scaleinvariance occurs automatically as long as the number of nuclei remains constant, even if the size of the embryo changes considerably [223, 83].

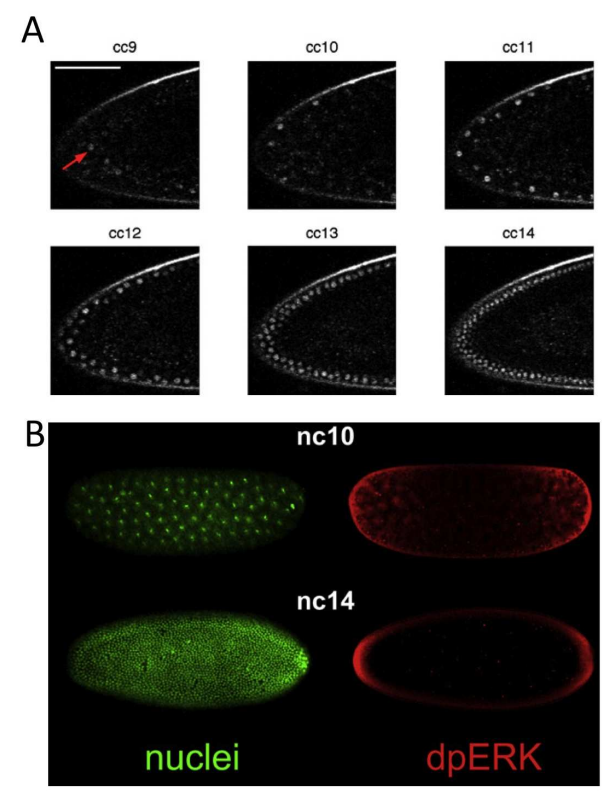

Figure 5: Nuclei are not passive observers of the morphogen gradient. (A) Bicoid is in dynamic equilibrium between the nuclei and the surrounding cytoplasm (from [83], with permission). (B) Nuclei shape the dpERK distribution in relation to their density and spatial localization (from [47], with permission). 


\subsection{BMP-mediated embryonic patterning}

Shortly after the discovery of Bicoid, the secreted morphogen Decapentaplegic (Dpp) was discovered in Drosophila. Dpp and Bicoid are similar in the sense that both are proteins forming dynamic, non-uniform distributions that ultimately specify differential gene expression over a field of naive cells, but this is where the similarities end. Bicoid is a transcription factor produced by mRNA that is maternally-deposited during oogenesis, and it is transported directly into the nuclei that line the periphery of the syncytial embryo. In contrast, Dpp is a zygotically-produced member of the bone morphogenetic protein (BMP) family that is secreted into the extracellular space, is controlled by numerous extracellular regulators, and must bind to transmembrane receptors that initiate a series of downstream steps that ultimately lead to target gene expression.

Dorsal surface patterning of the Drosophila embryo depends upon the activity of a heterodimer of BMP ligands Decapentaplegic (Dpp) and Screw (Scw) [203]. Screw is produced in all nuclei, whereas Dpp is produced along the dorsal half of the embryo. While monomers of both Dpp/Dpp and $\mathrm{Scw} / \mathrm{Scw}$ are present, the $\mathrm{Dpp} / \mathrm{Scw}$ heterodimer has $\approx 10$-fold higher activity and is probably the principal ligand for patterning. Dpp/Scw is secreted into the thin, fluid-filled perivitelline (PV) space surrounding the embryo. The BMP ligand transduces its signal by binding to type I BMP receptors Thickveins (Tkv) and Saxaphone (Sax) and type II BMP receptors Punt to form a heterotetrameric active signaling complex [203]. The activated receptor is a kinase that phosphorylates the intracellular molecule Mad to form pMad. pMad binds to two co-SMADs (Medea) and forms an active transcription factor that accumulates in the nucleus to regulate target gene expression.

If $\mathrm{Dpp} / \mathrm{Scw}$ secretion and receptor binding were the only processes occurring, one would expect that the spatial distribution of pMad would encompass nearly the entire region where $\mathrm{Dpp} / \mathrm{Scw}$ is produced. Instead, pMad begins low and broad, consistent with uniform receptor binding throughout the Dpp/Scw secretion zone, but later the pMad signal simultaneously intensifies and contracts from the broad early expression to form a narrow, high-intensity band centered at the dorsal midline, as shown in Figure 6. Dorsal surface patterning by BMPs requires the activity of at least 4 additional secreted molecules that control various aspects of the morphogen dynamics, range, and robustness (for a review, see [158]). The secreted BMP ligand is regulated by the secreted inhibitor Short gastrulation (Sog), the secreted co-factor Twisted gastrulation (Tsg), and the metalloprotease Tolloid (Tld) (see Figure 6). Tsg is produced along the dorsal surface in a band that covers the central $1 / 3$ of the embryo centered between the anterior and posterior poles. Sog is produced laterally in regions that flank the secretion zone of Dpp/Scw. The metalloprotease Tld is roughly constant throughout the dorsal half. The prevailing picture is that laterally-secreted Sog binds dorsally-secreted Tsg to form a potent inhibitor of Dpp/Scw. Dpp/Scw binds to Sog/Tsg, and the complex cannot bind to receptors but is free to diffuse. Tld then cleaves Sog specifically when it is in complex with Tsg and Dpp/Scw. The cleavage of Sog releases Dpp/Scw. The binding of $\mathrm{Dpp} / \mathrm{Scw}$ to Sog/Tsg is highest where the product of Dpp/Scw and Sog/Tsg concentrations is highest (near the Sog and Dpp/Scw secretion interface) and lowest near the dorsal midline where the balance between Tld processing and formation of $\mathrm{Dpp} / \mathrm{Scw} / \mathrm{Sog} / \mathrm{Tsg}$ favors the greatest release of Dpp/Scw. 


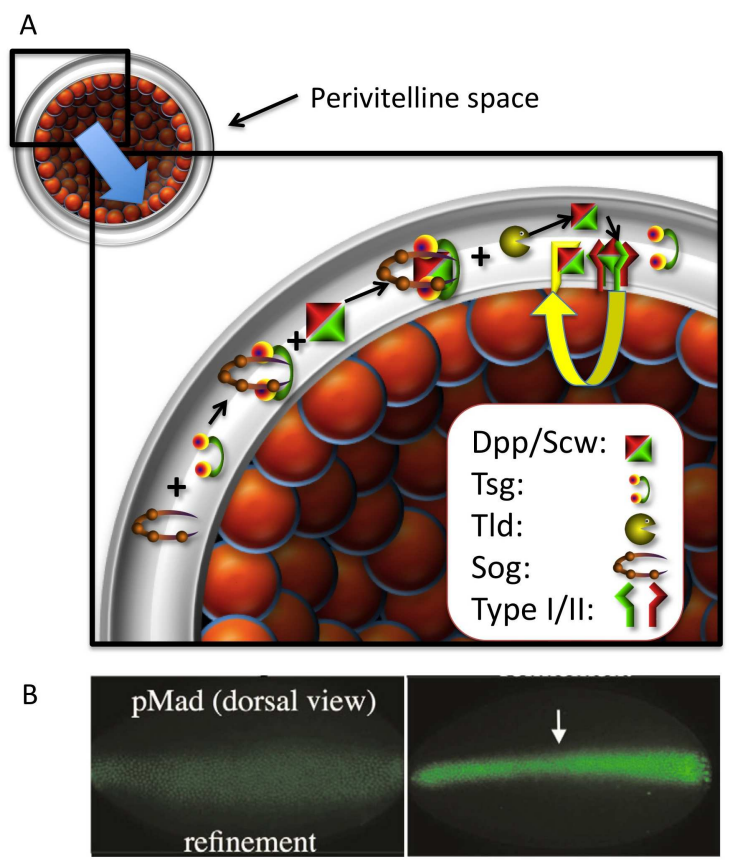

Figure 6: (A) BMP ligands and regulators are secreted into the thin, fluid-filled perivitelline space surrounding the embryo. The cross-sectional view of the syncytial embryo shows the chemical reactions that take place to localize BMP signaling to the dorsal surface. (B) The contraction of pMad expression with time. (From [224]. Copyright 2006 National Academy of Sciences, U.S.A.)

The model equations for Dpp/Scw-mediated patterning with positive feedback developed in [224] are as follows:

$$
\begin{aligned}
\frac{\partial B}{\partial t} & =D_{B} \nabla^{2} B+\phi_{B}(\mathbf{x})-k_{3} I \cdot B+k_{-3} I B+\lambda T l d \cdot I B \\
& -k_{4} B \cdot C+k_{-4} B C-k_{5} B \cdot R+k_{-5} B R \\
\frac{\partial S}{\partial t} & =D_{S} \nabla^{2} S+\phi_{S}(\mathbf{x})-k_{2} S \cdot T+k_{-2} I \\
\frac{\partial T}{\partial t} & =D_{T} \nabla^{2} T+\phi_{T}(\mathbf{x})-k_{2} S \cdot T+k_{-2} I+\lambda T l d \cdot I B-\delta_{T} T \\
\frac{\partial I}{\partial t} & =D_{I} \nabla^{2} I+k_{2} S \cdot T-k_{-2} I-k_{3} I \cdot B+k_{-3} I B \\
\frac{\partial I B}{\partial t} & =D_{I B} \nabla^{2} I B+k_{3} I \cdot B-k_{-3} I B-\lambda T l d \cdot I B
\end{aligned}
$$




$$
\begin{aligned}
\frac{\partial C}{\partial t} & =\frac{\Lambda B R^{\nu}}{K_{h}^{\nu}+B R^{\nu}}-k_{4} B \cdot C+k_{-4} B C-k_{7} B R \cdot C+k_{-7} B C R-\delta_{E} C \\
\frac{\partial B C}{\partial t} & =k_{4} B \cdot C-k_{-4} B C-k_{6} B C \cdot R+k_{-6} B C R-\delta_{E} B C \\
\frac{\partial B C R}{\partial t} & \left.=k_{6} B C \cdot R+k_{7} B R \cdot C\right]-k_{-6} B C R-k_{-7} B C R-\delta_{E} B C R \\
\frac{\partial B R}{d t} & =k_{5} B \cdot R-k_{-5} B R+k_{-7} B C R-k_{7} B R \cdot C-\delta_{E} B R \\
R_{\text {tot }} & =R+B R+B C R .
\end{aligned}
$$

Here, B is the BMP dimer (Dpp/Scw), S is Sog, T is Tsg, I is the Sog/Tsg inhibitor complex, IB is the complex when bound to the BMP ligand, $\mathrm{R}$ is the receptor level, and $\mathrm{C}$ is the level of secreted BMP binding protein. As can be seen from the preceding equations, the regulation of BMPs requires many non-linear steps that work in concert to produce BR distributions that correspond with our pMad observations.

The complex regulation of BMP signaling has been the focus of numerous mathematical modeling studies. Unlike the mathematical models used to measure aspects of Bicoid and dpERK patterning, the focus of the modeling of BMP patterning has been on discovering modules that satisfy a number of performance objectives $[115,61,138,224,223,203]$. The general idea behind this approach is that while we cannot measure specific conditions such as kinetic rates, transport rates, and molecular interactions in vivo, we can develop models that incorporate a number of plausible possibilities and use large-scale parameter screens to identify networks that reproduce features that are also observed in the biological system. For instance, in BMP-mediated embryonic patterning, some of the performance objectives that models were tested against include: 1) a sharp, dorsally-centered distribution of BMP ligands bound to receptors, 2) insensitivity to reductions in the levels of extracellular regulators, 3) correct spatiotemporal evolution of signaling, 4) insensitivity to increases or decreases in receptor levels, and 5) robustness against changes in embryo size (scale-invariance). The first modeling study on BMP-mediated dorsal surface patterning used a large-scale screen to identify model parameters that 1) produced a sharp BMP distribution, and 2) reproduced the experimentally observed robustness against genetic reductions in $s o g, s c w, t s g$, and tld. Eldar et al. used the results of their large-scale screen to predict that Scw and Dpp diffusion rates must be extremely slow unless it was bound by the inhibitor Sog, and they tested this by staining for pMad signaling in eve $2>d p p, s o g^{-/-}$embryos. Without the inhibitor Sog, they found that pMad signaling was restricted to the site of Dpp production. Following this study, Mizutani et al. suggested that Dpp was actually highly diffusible, but in the presence of receptors, the range of Dpp diffusion is very low [138]. (For a review, see [158]).

Following the initial modeling work that established the plausibility of the shuttling mechanism, further experiments demonstrated that the extracellular transport of Dpp/Scw formed only a piece of the puzzle, and that positive feedback as a result of prior BMP signaling was also required for the dynamic refinement and localized spatial distribution of pMad signaling [231] (Figure 6). Upon further investigation of the Eldar et al. and Mizutani et al. models with constant and uniform BMP production (Eldar et al. also considered a special case where Dpp production is turned off after 10 minutes, however this is demonstrably false [203]), it was found that neither model 
captured the appropriate dynamics of pMad signaling [61, 138, 224]. During development, pMad begins broad and low and refines to a narrow, high intensity band just prior to gastrulation (Figure 6). Both models predict that BMP-receptor (proportional to pMad) levels begin broad and low and, as time progresses, that the signal gets stronger and wider. Taking the new biological information and dynamics into consideration, Umulis et al. [224] developed a modular-based model to compare a number of different receptor/feedback mechanisms. In this study, the additional performance objectives included were that 1) the model produce the correct dynamics, and 2) the level of BMP-receptor complexes is insensitive to reductions or increases in the number of receptors as experimentally observed [231, 224]. The additional analysis suggested that positive feedback of a membrane-associated BMP binding protein such as Crossveinless-2 (Cv-2) yields the correct temporal dynamics, spatial distribution, and robustness of BMP signaling [224] (Figure 7 A-D). With the inclusion of positive feedback coupled with endocytosis in the BMP-patterning model, a new type of BMP-signal interpretation emerged: the dynamics of pMad signaling suggest that cells are dynamically responding to the extracellular distribution and competing for a limited number of BMP ligands [224]. The dynamic interpretation of BMP signaling not only reproduces the correct spatiotemporal signaling during development, but also produces very sharp boundaries between high and low signaling cells due to bistability. In the dynamic evolution to equilibrium, dorsally localized cells outcompete adjacent lateral cells for limited BMP ligands, thus reinforcing the difference between high and low pMad signaling (Figure 7 A- bottom panel).

Positive feedback can also lead to hysteresis which suggests that the concentration of BMPs is less important than the exposure of BMPs to different levels of signaling (Figure 7 B). An additional performance objective that is gained by inclusion of positive feedback is the insensitivity of signaling to the number of receptors. Both increases and decreases in the number of BMP receptors lead to pMad distributions that are virtually indistinguishable from $w t$ (Figure $7 \mathrm{C}$ ). To demonstrate the increased robustness against receptor levels, Umulis et al. [224] compared three different receptor models: 1) rapid equilibration, 2) receptor-mediated endocytosis, and 3) positive feedback of a secreted binding protein (Figure $7 \mathrm{D}$ ). With positive feedback, the modeling suggests that receptor levels can vary from less than $100 \mathrm{nM}$ to greater than $5 \times 10^{3} \mathrm{nM}$ concentrations and still produce the desired level of cells receiving high levels of BMP signaling. While the positively regulated molecule that controls pMad dynamics during embryogenesis has not been found, the recent discovery of the role of collagen in the embryo provides a new pathway of potential targets [230]. Briefly, Wang et al. [230] found that type IV collagen plays an important role in complex assembly between Dpp/Scw and the secreted inhibitors. Since modeling suggests that the positive feedback molecule must be localized where it is produced, it is possible that type IV collagen also plays a role in restricting the movement of other secreted BMP binding molecules.

In addition to embryonic patterning, a related model has been used to investigate the positive feedback of BMP signaling in a completely different patterning context. Here BMP patterning occurs during the development of crossveins in the Drosophila wing [200]. Wing patterning relies upon a set of secreted regulators homologous to those used for embryonic patterning, but it also requires the activity of an autonomous BMP binding protein called $\mathrm{Cv}-2$. $\mathrm{Cv}-2$ is involved in many different contexts and species as a positive and/or negative regulator of BMP signaling. In this context, local models were developed to identify biologically testable conditions that switch 


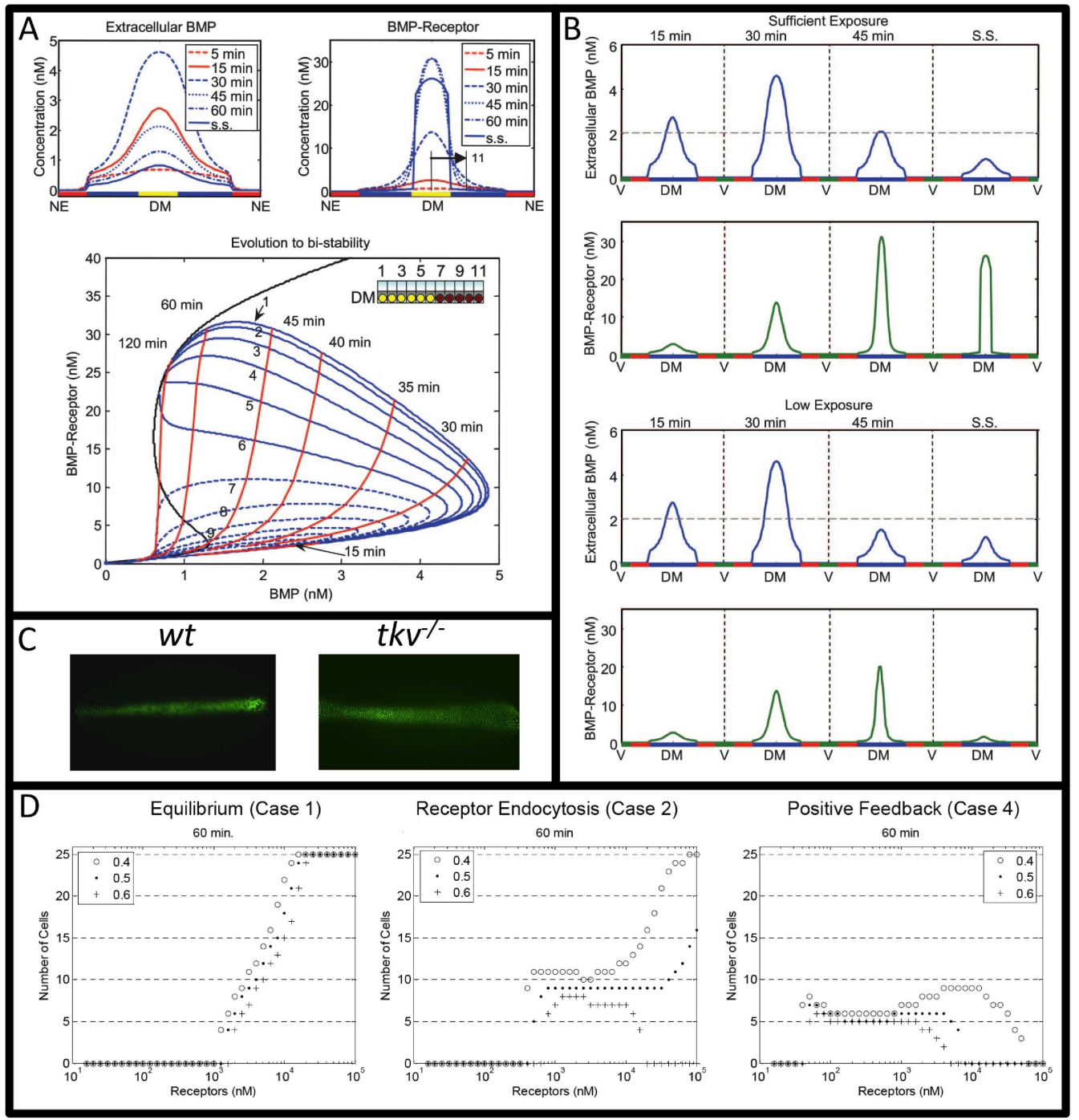

Figure 7: Mathematical modeling results for dorsal surface patterning. (A) Positive feedback leads to dynamic contraction of BMP-receptors and bistability which leads to the sharp boundary between high and low signaling states. (B) Hysteresis leads to a dynamic interpretation of extracellular BMP in the positive feedback model. (C) pMad staining in $w t$ and tkv mutant embryos is nearly identical. (D) The model with positive feedback is less sensitive to changes in receptor levels than models other models of receptor regulation. (From [224]. Copyright 2006 National Academy of Sciences, U.S.A.)

$\mathrm{Cv}-2$ from being an activator to an inhibitor of BMP signaling. According to the modeling, using a low affinity ligand and changing the concentration of $\mathrm{Cv}-2$ impart the biphasic behavior of $\mathrm{Cv}-2$ regulation. The model predictions were later tested and verified both in vivo and in vitro; additional details can be found in [200]. 


\section{Bacterial pattern formation}

Bacteria are single cell organisms that proliferate through binary fission. Generally of the order $\sim$ $1-10 \mu \mathrm{m}$, they adapt to a wide variety of environments and, while many are pathogenic, form vital symbiotic relationships with many higher-level organisms (for example, in the digestive system). The capacity to move is an essential trait in many bacteria that allows them to migrate towards more favorable environments or away from more harmful environments in response to extracellular signals such as chemicals, light and oxygen. Of these, the tendency to follow extracellular chemical gradients (chemotaxis) has been widely studied for its critical role in coordinating multicellular behavior.

Many bacteria do not live as individuals, but rather form multicellular colonies that communicate through chemical signals [201]. Intercellular communication leads to the formation of biofilms, communities of one or more bacterial types enclosed by self-secreted extracellular matrices [234, 53, 229, 80, 112]. Biofilms form almost anywhere a nutrient supply is available and surface attachment is possible, for example, plaques on teeth and slippery coatings on stones. Initiation of biofilms involves random attachment of planktonic cells to surfaces, and subsequent growth is facilitated by chemotactic movement toward the colonizers, which thereby increases the attachment probability. Mature biofilms form channels that ensure the efficient delivery of nutrients from exterior to interior.

Depending on the context, biofilms can be either harmful or beneficial. On teeth, on artificial medical implants, and in chronic wounds the formation of biofilms is harmful. On the other hand, biofilms play important roles in bioremediation technology, allowing chemical waste to be degraded in water [209]. The chemotaxis of bacterial cells towards contaminants has the potential to bring cells into close proximity to waste, thereby facilitating the degradation process. Understanding the mechanisms controlling biofilm formation and function may therefore lead to improved modes for eliminating harmful bacteria and improving their beneficial effects.

The spectacular growth patterns observed in a variety of bacterial colonies [34, 35, 237, 20, $17,26,189]$ provide a simple and manipulable system for studying the initiation of biofilms. The fundamental processes of proliferation and movement conspire to generate diverse pattern forms, and understanding their development will allow us to exploit biofilm properties to treat biofilm induced disease or increase the effectiveness of bioremediation. In this section we review both the biological experiments and mathematical models for the patterns formed in Escherichia coli colonies. Pattern formation in other bacterial colonies, e.g. Bacillus, Proteus and Myxococcus is discussed in $[18,238,101,102]$ and references therein.

\subsection{Pattern formation in $E$. coli colonies}

Escherichia coli is a cylindrical enteric bacterium that swims using a run-and-tumble strategy $[22,23,228]$. Each cell possesses 5-8 helical flagella extending several body lengths, with each flagellum capable of rotation clockwise $(\mathrm{CW})$ or counterclockwise $(\mathrm{CCW})$, driven by its basal rotary motor. When rotated CCW the flagella form a smooth bundle to propel the cell forward in a smooth "run" with speeds between 10 and $30 \mu \mathrm{m} / \mathrm{s}$; when rotated CW the bundle flies apart, the 
cell stops almost immediately due to the low Reynolds number $\left(\sim 10^{-6}\right)$, and "tumbles" randomly. Each tumble reorients the cell in a quasi-random direction, although there is a slight bias in the direction of the previous run [86]. In the absence of signal gradients, the alternation between running and tumbling results in random cell movement, and run/tumble times are exponentially distributed with means of 1 and 0.1 seconds, respectively. However, when exposed to a signal gradient run times are extended when moving up (down) a chemoattractant (chemorepellent) gradient, thereby biasing cell movement.

The molecular mechanism of chemotaxis in E. coli has been studied extensively [123, 228, 29]. The signal transduction pathway contains three basic elements, as is shown schematically in Figure 8. The first is the detection of the signal by chemoreceptors. E. coli has five chemoreceptor types, four of which are methyl-accepting chemotaxis proteins (MCPs). MCPs form homodimers that span the bacterial cell membrane, and these assemble into trimers of dimers, which are the functional unit of MCPs. The cooperativity of the functional units contribute one part of the high gain of the chemotaxis system. The second is the transduction of the signal, which involves a decrease in autophosphorylation of and phosphotransfer to chemotaxis proteins. Attractant binding to a receptor reduces the autokinase activity of the associated CheA, and therefore reduces the level of phosphorylated $\mathrm{CheY}_{\mathrm{p}}$, which is the output of the transduction network, on a fast time scale $(\sim 0.1 \mathrm{~s})$. This constitutes the excitation component. Changes in the methylation level of the receptor by $\mathrm{CheR}$ and $\mathrm{CheB}$ restores the activity of the receptor complex to its pre-stimulus level on a slow time scale (seconds to minutes). This results in adaptation to the ambient signal, which enables the cell to respond to further signals. The third component is the change in bias of the rotation of flagellar motors due to changes in $\mathrm{CheY}_{\mathrm{p}}$. The default rotation of the motor is $\mathrm{CCW}$, which leads to smooth swimming. When $\mathrm{CheY}_{\mathrm{p}}$ binds to the switch protein FliM on the flagellar motor, the motor has a higher probability to rotate $\mathrm{CW}$. The ultrasensitivity of the motor to $\mathrm{CheY}_{\mathrm{p}}$ contributes to the remaining part of the high gain. The concerted motion of several flagellar motors enables the cell to swim. Therefore increasing attractant concentration leads to decreasing CheY and therefore a longer run time on average.

Chemotaxis in E. coli plays a major role in spontaneous, self-organized pattern formation, primarily when factors such as the nutrient supply vary in space. Experiments by Adler in the 1960s led to the discovery of moving bands/rings for a bacteria colony exposed to a nutrient that also serves as a chemoattractant [1]. Budrene and Berg uncovered a wide spectrum of stable spatial pattern types generated by $E$. coli cells, attributed in part to the fact that the bacteria secrete chemoattractants (aspartate and analogues) [34, 35]. Similarly-derived patterns have also been observed in S. typhimurium colonies [237].

In the first type of experiment, a small number of $E$. coli cells was introduced at the center of a semi-solid agar substrate in the presence of a single nutrient source (succinate or other highly-oxidized intermediates of the TCA cycle). Over the following three days, a concentric swarm ring is observed to spread radially from the inoculation site (IS) with a symmetric array of spots or stripes forming sequentially in its wake (Figure 9). The initial dynamics are driven mainly through proliferation of the inoculation, with a doubling time of approximately 2 hours [35]. Succinate consumption induces the production of aspartate by the growing colony, leading to a chemotactically-driven compact aggregate at the IS of the order of $200 \mu \mathrm{m}$ diameter. Lo- 


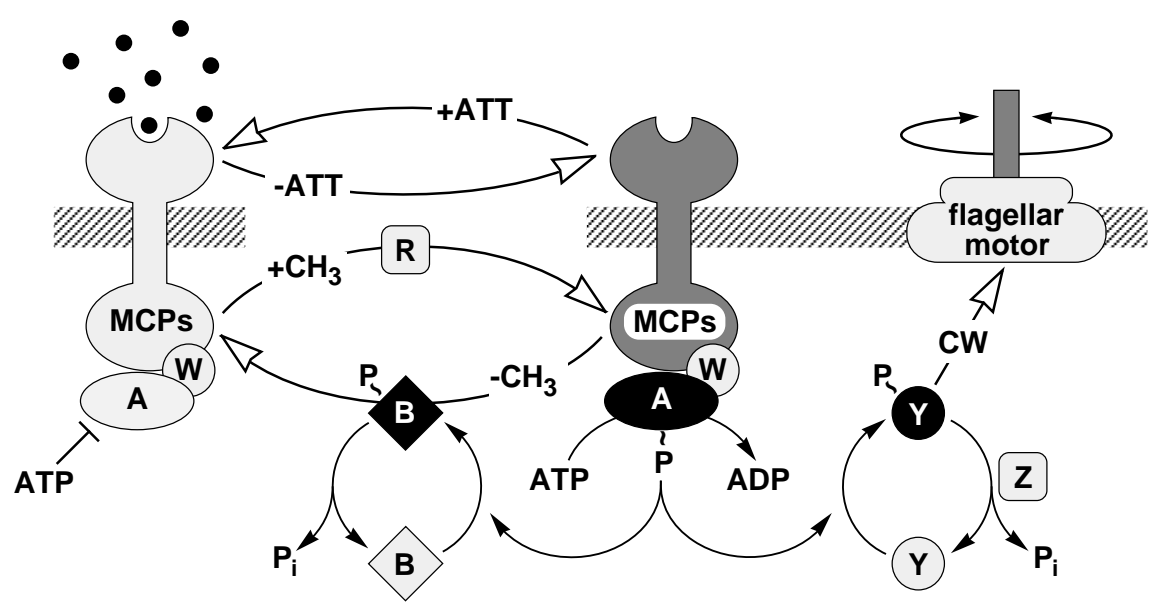

Figure 8: Signaling components and pathways for E. coli chemotaxis. Chemoreceptors (MCPs) span the cytoplasmic membrane (hatched lines), with a ligand-binding domain on the periplasmic side and a signaling domain on the cytoplasmic side. The cytoplasmic signaling proteins, denoted Che in the text, are identified by single letters, e.g., A = CheA. (From [210]. Copyright 1997 National Academy of Sciences, U.S.A.)

cal depletion of succinate leads to starvation and the cessation of aspartate secretion at its center, while cells at its boundary continue to produce aspartate due to succinate diffusing into the aggregate from the surrounding substrate. As these cells spread outwards, a positive aspartate gradient is generated that provides the directional information for centralized cells to keep pace with the front. A swarm ring thus develops that moves radially outwards with constant speed. Depending on the initial succinate concentration, aggregates may or may not form and stabilize in the wake of the swarm ring. At low concentrations of succinate the swarm ring may propagate to the edge of the petri dish without breaking up (Figure 9, a). At higher concentrations aggregates form inside the ring. The ring continues to move and the aggregates gradually detach from the ring. The cells in the aggregates finally become nonmotile, which stabilizes the pattern. The radial sequence of aggregates form by a repetition of the sequence that initiated the first generation of aggregates. If the concentration of succinate is not too high the aggregates are aligned radially (Figure 9, b). When the radius becomes large, new spokes of aggregates appear between the old ones. If the concentration is higher, new aggregates form between the preceding sites (Figure 9, c). The speed of the swarm ring is inversely proportional to the succinate concentration, as is the radius at which the ring collapses into spots.

A second set of experiments was performed by suspending $E$. coli cells in a liquid medium. Under sufficiently high cell densities, the chemotactic response to aspartate induces the cells to form networks initially, which subsequently collapse into moving aggregates which merge over time [24]. The timescale of patterning for the liquid suspension experiments is of the order of minutes and the role of proliferation can therefore be neglected. The properties of a single aggregate have been investigated in [137] for a quasi-2D system. The equilibrium size of aggregates, $150 \sim 200 \mu \mathrm{m}$ in diameter, was shown to depend only weakly on the density of cells and most probably on the sensory mechanism of the bacteria. The tumbling rate of a cell depends strongly 

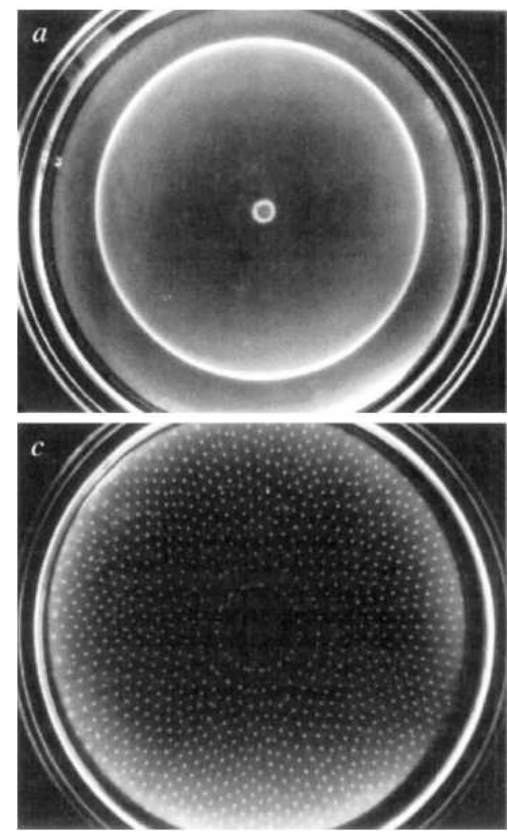
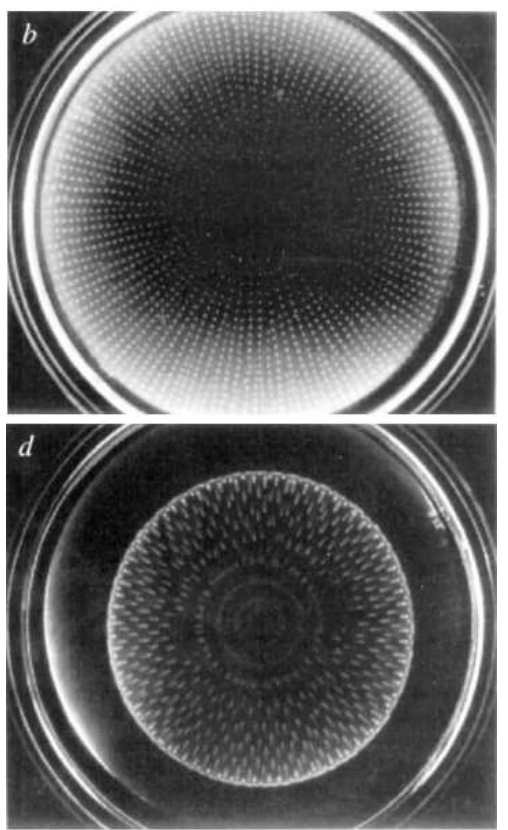

Figure 9: Patterns generated by E. coli cells cultured in semi-solid agar [35] under different succinate levels: (a) $1 \mathrm{mM}$, (b) $2 \mathrm{mM}$, (c) $3 \mathrm{mM}$, (d) $3 \mathrm{mM}$. The E. coli strains used in experiments are not chemotactic to serine and either have the gene tsr deleted (a,b,c, strain HCB317) or are serine-blind (d, strain RP4368). A similar pattern to that in (d) can be generated with the strain HCB317. The concentration of succinate required to generate a particular pattern differs for different strains, but the sequence of patterns with respect to succinate concentration remains the same. (From [35], with permission.)

on its position within the aggregate and the direction of movement: tumbling is suppressed when swimming towards the center of the cluster, resulting in overshoot, but when swimming at the periphery of the aggregate cells tumble immediately. These two responses conspire to maintain the boundary and fix the aggregate size.

The formation of these bacterial patterns involves a complex interplay between different processes, including consumption of nutrients, production of chemoattractants, tactic movement towards the attractant, cell growth and loss of motility. Moreover, formation of these spatial patterns can involve millions of cells. Mathematical models of the patterns include continuum models that incorporate these processess using phenomenological descriptions of the processes, and hybrid cell-based models that allow detailed description of microscopic behavior. The continuum models are easier to implement and more amenable to mathematical analysis, but justification of the models has to be addressed. Hybrid cell-based models give a better description of the experimental picture and allow for investigation to the balance of different processes, but can be expensive to implement. In the remainder of this section we first review both types of models that facilitated our understanding of the mechanisms underlying formation of the complex patterns, and we then summarize the current results on deriving the continuum model from the cell-based model. 


\subsection{Continuum models for bacterial pattern formation}

A variety of Patlak-Keller-Segel type systems, as described in Section 2.4., have been developed and applied to model E. coli and $S$. typhimurium patterns [106, 117, 118, 19, 218, 237, 221, 220, $222,185]$. In [106] it is shown that the system of equations

$$
\begin{aligned}
& \frac{\partial n}{\partial t}=\nabla \cdot\left(D_{n} \nabla n-\frac{\chi_{0}}{S} n \nabla S\right), \\
& \frac{\partial S}{\partial t}=D_{s} \triangle S-k S,
\end{aligned}
$$

allows for an analytic traveling wave solution, but the singular chemotaxis sensitivity chosen there violates the fact that cells have bounded traveling speed when $S$ is small. A nonsingular chemotactic sensitivity however would lead to a moving band with decreasing speed and broadening density profile due to diffusion. Later in $[117,118]$ it is shown numerically that by incorporating cell growth and death the speed and shape of the band can be stabilized.

To model the E. coli patterns published [34,35], the PKS model was extended to include both attractant and substrate. In Ben-Jacob et al. [19] and Tsimring et al [218] a second repellent field was assumed to autocatalyze the production of attractant. Simulations revealed radially aligned and sunflower-like aggregation patterns. However, the assumption of the repellent field was not biologically supported. In [221] a minimal model was proposed to account for patterns formed in both liquid and semi-solid media. The equations describe the dynamics of the cell density $n$, the attractant concentration $S$, and substrate (nutrient) concentration $f$ as follows,

$$
\begin{aligned}
\frac{\partial n}{\partial t} & =D_{n} \Delta n-\nabla \cdot\left(\frac{k_{1} n}{\left(k_{2}+S\right)^{2}} \nabla S\right)+k_{3} n\left(\frac{k_{4} f^{2}}{k_{9}+f^{2}}-n\right), \\
\frac{\partial S}{\partial t} & =D_{s} \triangle S+\frac{k_{5} f n^{2}}{k_{6}+n^{2}}-k_{7} n S \\
\frac{\partial f}{\partial t} & =D_{f} \triangle f-k_{8} n \frac{k_{4} f^{2}}{k_{9}+f^{2}} .
\end{aligned}
$$

Simulations under suitable parameter sets reveal aggregate and swarm patterns roughly agreeing with the experiments shown in Figure 9 (b)-(c). However no clear swarm rings with sharp boundary seem to form as in the semi-solid agar, nor does the network/stranded pattern which precedes the formation of aggregates seen in liquid medium experiments. The model was later analzsed under the condition $k_{3}=k_{7}=0$ corresponding to aggregate formation, merging and disappearance in liquid medium in [220]. Linear analysis around the spatially-uniform solution showed that the uniform steady state is always stable to large wave number perturbations but unstable to lower wave number perturbations for a limited amount of time, and that leads to the appearance and disappearance of the aggregates. The analysis agrees with the experiments and simulations qualitatively, but the index of the mode with the largest amplitude differs from the number of peaks in simulations by a factor of two. How the nonlinearity in the system contributes to this discrepancy is not clearly understood. Polezhaev et al. [185] proposed a model that also incorporates loss of motility of cells due to starvation. The resulting simulations revealed the formation of a swarm 
ring and stable aggregation patterns composed of immobile cells. However, the swarm ring formed has a very diffusive front, in contrast to what is observed in experiments. Although all the above models can produce patterns with some similarity to those in the semi-solid agar experiments, how different processes balance to shape the patterns was not addressed.

This was addressed by Brenner al. [31], who developed a model for the purpose of understanding which processes are responsible for different localized features of the patterns, e.g., swarm ring formation and aggregate formation and disassociation from the ring. The PKS chemotaxis equation was coupled to reaction-diffusion equations for attractants and nutrients and a minimal mechanism for the swarm ring and aggregate formation was proposed. The authors suggested that movement of the swarm ring was driven by local nutrient depletion, with its integrity resulting from the high attractant concentration at the ring location; in contrast, aggregates were proposed to form within the ring through fluctuations about the unstable uniform cell density. An asymmetric profile in the attractant concentration arises due to the consumption of aspartate by cells following the depletion of succinate. The results are qualitative due to the discrepancies between predictions of the linearized system and the full nonlinear system, and mechanisms for the regularity of the patterns in semi-solid agar were not considered. In a subsequent paper [25] an analysis of the transformation of high cell density cylinders into regularly spaced aggregates from the swarm ring was undertaken, with the conclusion that a shifting balance between diffusion and chemotaxis leads to collapse of the strands.

\subsection{Cell-based models of bacterial pattern formation}

Hybrid cell-based models have been developed for bacterial pattern formation in [62, 240, 238]. In these models, each cell is characterized by its position $\mathbf{x} \in \mathbb{R}^{N}$, velocity $\mathbf{v} \in V \subseteq \mathbb{R}^{N}$, internal state $\mathbf{y}$ and age $\theta$. In $E$. coli the cell speed is more or less constant throughout the movement, so we assume that only the direction of the velocity changes during a tumble, therefore $V$ becomes a sphere. In the experiments of Budrene and Berg [35], the cell density is $\mathcal{O}\left(10^{8}\right) \mathrm{ml}^{-1}$, thus the average volume fraction of the cell population in the substrate is $\mathcal{O}\left(10^{-4}\right)$. Even if in an aggregate cells are 100 times more crowded than average, the volume fraction would still be as small as $\mathcal{O}\left(10^{-2}\right)$. Therefore, it is plausible to assume that cells are well separated, and there is no mechanical interaction between them. This means that we can treat the movement of different cells as independent processes. Since the mean tumbling time $(\sim 0.1 \mathrm{~s})$ is much shorter than the run time $(\sim 1 \mathrm{~s})$, we also neglect the tumbling time and assume that cells reorient immediately. Therefore the chemotactic movement of each bacterial cell is an independent velocity jump process (see Section 2.4.). The turning rate and turning kernel are determined by the internal variables $\mathbf{y}$.

The signal transduction pathway of $E$. coli is very complicated, and several detailed mathematical models have been proposed for the entire signal transduction network [210, 202, 134, 188]. However the essential dynamics are simple and can be captured by a minimal cartoon model that incorporates both the adaptation and excitation processes crucial to chemotaxis [168]. It was shown in [64] that the following model predicts qualitatively and quantitatively similar input-output be- 
havior to that of the full model in [210]:

$$
\begin{aligned}
\frac{d y_{1}^{i}}{d t} & =\frac{G\left(S\left(\mathbf{x}^{i}, t\right)\right)-\left(y_{1}^{i}+y_{2}^{i}\right)}{t_{e}} \\
\frac{d y_{2}^{i}}{d t} & =\frac{G\left(S\left(\mathbf{x}^{i}, t\right)\right)-y_{2}^{i}}{t_{a}} .
\end{aligned}
$$

Here the superscript $i$ is the index of the cell, $S$ is the local attractant concentration and $t_{e}$ and $t_{a}$ (with $t_{e} \ll t_{a}$ ) are constants defining excitation and adaptation timescales. The function $G(S)$ models the detection of the extracellular (chemoattractant) signals. We adopt this cartoon model and suppose that the turning kernel $T$ and turning rate $\lambda$ are given by

$$
T=\frac{1}{|V|} \quad \text { and } \quad \lambda^{i}=\lambda_{0}\left(1-\frac{y_{1}^{i}}{\gamma_{0}+\left|y_{1}^{i}\right|}\right)
$$

where

$$
\int_{V} T\left(\mathbf{v}, \mathbf{v}^{\prime}\right) d \mathbf{v}=1
$$

This cell-based description for chemotactic movement can be augmented with reaction-diffusion equations to describe dynamics of the extracellular nutrient and attractant and numerically solved with a hybrid scheme: the movement of each cell is simulated by a Monte Carlo method while the reaction-diffusion equations are solved with an alternating direction method. The details of this algorithm can be found in [240]. In the following we present numerical results in a 2-D domain. Although we do not expect significant differences, extension of the results to 3-D is currently under investigation.

\subsubsection{Network and aggregate formation in $E$. coli liquid medium}

To model the liquid medium experiments, E. coli cells are distributed evenly in a $3 \mathrm{~cm} \times 3 \mathrm{~cm}$ square under a uniform initial nutrient supply and zero initial attractant. The dynamics for the attractant and nutrient concentrations are described by

$$
\begin{gathered}
\frac{\partial S}{\partial t}=D_{s} \triangle S+\gamma \sum_{n=1}^{N} \delta\left(\mathbf{x}-\mathbf{x}^{i}\right)-\mu S . \\
\frac{\partial f}{\partial t}=D_{f} \Delta f-k \sum_{n=1}^{N} \delta\left(\mathbf{x}-\mathbf{x}^{i}\right) .
\end{gathered}
$$

Here $D_{s}$ and $D_{f}$ are the diffusion rates, $\gamma$ defines the attraction secretion rate by cells and $\mu$ is an unspecified degradation rate. The experimental time for liquid medium experiments is between 10 and 15 minutes and proliferation/death is therefore negligible. Furthermore, in an abundant supply the nutrient equation can be dropped. Figure 10 shows the evolution of cell density, first into a network of high cell density, then breaking up into randomly-distributed aggregates. These clusters remain motile and a cluster close enough to another merges with it. Moreover the cluster 



Figure 10: Simulation showing the formation of networks and aggregates for the suspension of $E$. coli in a liquid medium using the hybrid model defined by equations (4.5-4.9). Parameters used: $L=3 \mathrm{~cm}, f_{0}=0.3$ $\mathrm{mM}, \bar{s}=10 \mu \mathrm{m} / \mathrm{s}, \lambda=1 \mathrm{~s}^{-1}, D_{s}=9 \times 10^{-6} \mathrm{~cm}^{2} / \mathrm{s}, \mu=0, \omega=10^{-5} \mathrm{~s}^{-1}, \gamma_{0}=5 \times 10^{-4}, t_{a}=5 \mathrm{~s}, G(S)=S$.

size is in good agreement with experimental measurements. This model is the first to predict network formation and the conclusions are robust, since altering the total number of cells has a negligible effect on the size of individual aggregates, but of course does change the total number of aggregates.

\subsubsection{Expanding swarm ring in E. coli semi-solid agar}

The hybrid model was applied to model the semi-solid agar experiments under the assumptions of [31]. It is assumed that cells consume succinate and secrete aspartate at a rate proportional to the extracellular succinate concentration. A low succinate concentration is assumed to induce cells to consume aspartate [31]. Following the introduction of a small population of bacteria, a cell colony is observed to evolve into a ring of bacteria which expands radially at constant speed $(\approx 2 \mathrm{~mm} / \mathrm{h})$, as shown in Figure 11. The aspartate concentration echoes the bacteria profile while the succinate 
is rapidly depleted by the colony, forming a sharp gradient. The sharp wave front predicted here is in agreement with experiments.

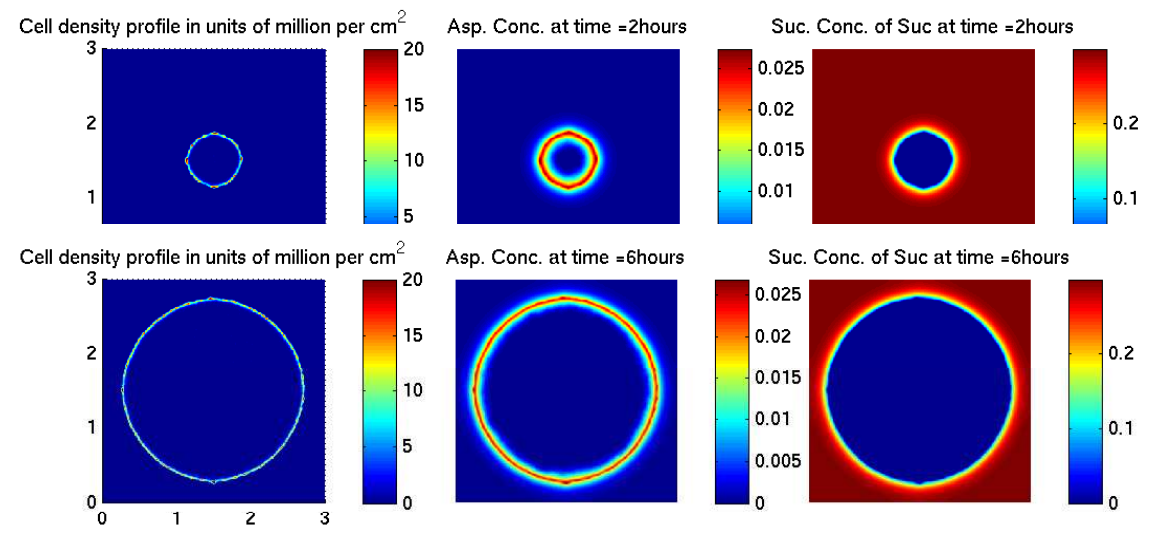

Figure 11: Simulations showing an expanding swarm ring for an E. coli colony inoculated in the semi-solid agar. Parameters used: $L=3 \mathrm{~cm}, f_{0}=0.3 \mathrm{mM}, \bar{s}=10 \mu \mathrm{m} / \mathrm{s}, \lambda=1 \mathrm{~s}^{-1}, D_{s}=9 \times 10^{-6} \mathrm{~cm}^{2} / \mathrm{s}, \omega=10^{-5}$ $\mathrm{s}^{-1}, \gamma_{0}=5 \times 10^{-4}, t_{a}=5 \mathrm{~s}$, starving delay $=20 \mathrm{~min}$.

In Figure 12 the sensitivity of the chemotaxis mechanism is increased by 10 fold $\left(\gamma_{0}=5 \times\right.$ $10^{-5}$ ). Inhomogeneities inside the swarm ring lead to the formation of a polygon pattern with fast-moving high-density aggregates and connecting catenary arcs. The pattern arises from the fact that the aggregates consume succinate at a higher rate and therefore move with a larger speed than the arcs. Notably, this pattern was also observed in certain species of $E$. coli in unpublished data [33]. The disassociation of aggregates from the swarm ring is currently the subject of further investigations; a possible driving mechanism is the high aspartate concentration in the swarm ring saturating the cells and impeding their movement towards regions of higher nutrient. How different parameters in saturation lead to the different patterns of aggregation formation or polygon patterns remains to be investigated.
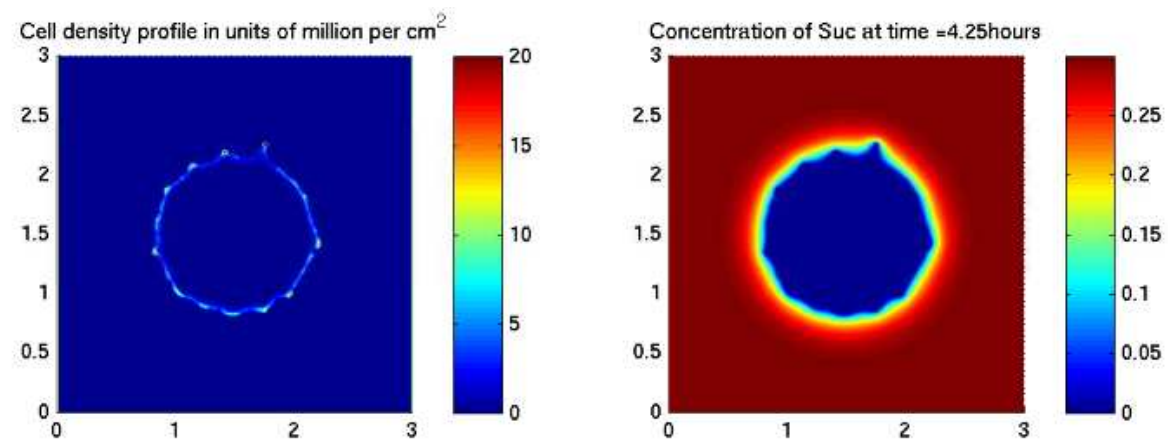

Figure 12: Simulated polygon formation in an E. coli colony. 


\subsection{From cell to continuum}

As discussed earlier, a classical PKS approach has been employed to model bacterial pattern formation by a number of authors $[19,218,220,221,185]$. However questions remain as to whether the PKS equation is a justifiable limit of the underlying microscopic process. Following the introduction of velocity jump processes to characterize the movement of bacterial cells in [165], significant progress has been made in determining the conditions under which the PKS equation can be derived $[89,166,64,63,240]$.

The transport equation for the classical velocity jump process described in Section 2.4. is

$$
\frac{\partial}{\partial t} p(\mathbf{x}, \mathbf{v}, t)+\mathbf{v} \cdot \nabla p(\mathbf{x}, \mathbf{v}, t)=-\lambda p(\mathbf{x}, \mathbf{v}, t)+\lambda \int_{V} T\left(\mathbf{v}^{\prime}, \mathbf{v}\right) p\left(\mathbf{x}, \mathbf{v}^{\prime}, t\right) d \mathbf{v}^{\prime}
$$

where $p(\mathbf{x}, \mathbf{v}, t)$ is the probability density of a cell at position $\mathbf{x}$ with velocity $\mathbf{v}$ at time $t \geqslant 0$, $\lambda$ is the turning rate of the cell and $T\left(\mathbf{v}^{\prime}, \mathbf{v}\right)$ is the probability density of jumping between $\mathbf{v}^{\prime}$ and $\mathbf{v}$ at a "tumble". Note that conservation of cells implies $\int_{V} T\left(\mathbf{v}^{\prime}, \mathbf{v}\right) d \mathbf{v}=1$. Under the assumption of independent and identical cells, $p$ can be also viewed as the cell density. Under reasonable assumptions on the turning kernel, the diffusion limit of the transport equation (4.10) was investigated in [89] and equation (2.16) was derived using regular perturbation analysis. In general, the diffusion 'coefficient' $D_{n}$ is a second-rank tensor, and conditions under which $D_{n}$ is diagonal were determined [89]. In the presence of a chemical signal, the classical PKS chemotaxis equation (2.18) was derived under the assumption that the extracellular signal field $S$ enters via the functions $\lambda$ and $T$ [166].

To understand how the chemotactic sensitivity depends on parameters of the intracellular dynamics, Erban and Othmer incorporated the velocity jump process with the internal state variables, $\mathbf{y}[64,63]$. The transport equation becomes

$$
\frac{\partial p}{\partial t}+\nabla_{x} \cdot(\mathbf{v} p)+\nabla_{y} \cdot(\mathbf{f} p)=-\lambda(\mathbf{y}) p+\int_{V} \lambda(\mathbf{y}) T\left(\mathbf{v}, \mathbf{v}^{\prime}, \mathbf{y}\right) p\left(\mathbf{x}, \mathbf{v}^{\prime}, \mathbf{y}, t\right) d \mathbf{v}^{\prime} .
$$

Applying moment closure techniques and regular perturbation analysis, the authors obtained the equation

$$
\frac{\partial}{\partial t} n=\nabla \cdot\left[\frac{s^{2}}{N \lambda_{0}} \nabla n-G^{\prime}(S(x, t)) \frac{b s^{2} t_{a}}{N \lambda_{0}\left(1+\lambda_{0} t_{a}\right)\left(1+\lambda_{0} t_{e}\right)} n \nabla S\right],
$$

first derived in $1 \mathrm{D}$, and later extended to $3 \mathrm{D}$ on the diffusion time and space scales [64, 63]. A major assumption in this derivation is that $\frac{b}{\lambda_{0}} G^{\prime}(S) \nabla S \cdot \mathbf{v} \sim \mathcal{O}(\epsilon) \mathrm{sec}^{-1}$, i.e., the signal gradient is shallow. Other assumptions include time-independent signals $S=S(\mathbf{x})$ and a linear turning rate $\lambda=\lambda_{0}-b y_{1}$. The derivation was generalized in [240] to admit a time dependent signal and nonlinear dependence of the turning rate on internal variables. The shallow gradient assumption therefore becomes $H:=\frac{a_{1}}{\lambda_{0}} G^{\prime}(S)\left(\nabla S \cdot \mathbf{v}+\frac{\partial S}{\partial t}\right) \sim \mathcal{O}(\epsilon) \sec ^{-1}$, where $a_{1}=-\frac{\partial \lambda}{\partial y_{1}}$. In addition, the analysis was further generalized by considering external forces. As an example, treating the swimming bias that arises when cells are near a surface ( which leads to the spiral density patterns seen in $P$. mirabilis ) as an external force, leads to the equation

$$
\frac{\partial}{\partial t} n=D_{n} \Delta n-\nabla \cdot\left[G^{\prime}(S) n\left(\chi_{0} \nabla S+\beta_{0}(\nabla S)^{\perp}\right)\right]
$$


under the assumption of fast excitation $\left(t_{e}=0\right)$. Here

$$
\begin{aligned}
D_{n} & =\frac{s^{2}}{2 \lambda_{0}\left(1-\psi_{d}\right)+\frac{2 \omega_{0}^{2}}{\lambda_{0}\left(1-\psi_{d}\right)}} \\
\chi_{0} & =\frac{a_{1} s^{2}\left(1-\psi_{d}\right)\left[\lambda_{0}\left(1-\psi_{d}\right)\left(\lambda_{0}\left(1-\psi_{d}\right)+\frac{1}{t_{a}}\right)-\omega_{0}^{2}\right]}{2\left(\left(\lambda_{0}\left(1-\psi_{d}\right)+\frac{1}{t_{a}}\right)^{2}+\omega_{0}^{2}\right)\left(\lambda_{0}^{2}\left(1-\psi_{d}\right)^{2}+\omega_{0}^{2}\right)} \\
\beta_{0} & =\frac{\omega_{0} a_{1} s^{2}\left(1-\psi_{d}\right)\left(2 \lambda_{0}\left(1-\psi_{d}\right)+\frac{1}{t_{a}}\right)}{2\left(\left(\lambda_{0}\left(1-\psi_{d}\right)+\frac{1}{t_{a}}\right)^{2}+\omega_{0}^{2}\right)\left(\lambda_{0}^{2}\left(1-\psi_{d}\right)^{2}+\omega_{0}^{2}\right)}
\end{aligned}
$$

The parameter $\omega_{0}$ measures the swimming bias, while $\psi_{d}$ is the index of directional persistence (see [239] for details).

The ultimate aim is to derive equations for the cell density from the cell-based model under a variety of signal gradients. In [238] it is shown that for an ultra-small signal gradient, $H \leq$ $\mathcal{O}\left(\epsilon^{2}\right) \sec ^{-1}$, the chemotactic response of the population enters into the cell density solution as small perturbations (higher order terms) to a diffusion process $\left(n^{0}\right)$ with $D_{n}=s^{2} /\left(N \lambda_{0}\right)$, and the chemotaxis equation (4.12) still provides a good approximation. For larger signal gradients, $H \geq \mathcal{O}(1) \mathrm{sec}^{-1}$, however, Equation (4.12) may fail to provide a reasonable approximation, since the macroscopic velocity

$$
\mathbf{u}_{S}=\chi(S) \nabla S=s\left(\frac{a_{1} s}{\lambda_{0}} G^{\prime}(S) \nabla S\right)\left(-\frac{1}{|V|} \int_{V} \mathbf{v} \otimes\left(t_{a} \lambda_{0} \mathcal{A}-1\right)^{-1} \mathbf{v} d \mathbf{v}\right)
$$

can increase beyond realistic cell speeds when $\nabla S$ is large. Exactly how to derive appropriate macroscopic equations from the microscopic process and what equation to expect for larger signal gradients remains an open problem. Related unresolved issues arise when one tries to derive the PKS equation for amoeboid cells [65].

\section{Pigmentation markings}

Pigmentation in vertebrates initiates early in the embryo following the migration of pigment cell precursors from their neural crest origin to the dermal skin layers [119]. At the time at which the pattern is laid down, each stripe of the zebra or spot of a leopard is of the order of 100 pigment cells in diameter, and the question naturally arises as to what mechanisms are involved in the large scale organization of cells to produce a particular pattern within the skin. The study of pigmentation patterning is thus an elegant model system for exploring the fundamental processes governing embryonic morphogenesis. In particular, pigment patterning provides a rare opportunity to examine the coupling between embryonic growth and morphogenetic patterning, because for certain species (particularly fish) the pigmentation pattern evolves with organism growth throughout the individual's lifespan.

Despite the many differences at the microscopic level, for example the number of pigment cell types and the mode by which pigment is transferred, the same basic pattern types - stripes 
and spots - are widespread across the animal kingdom, raising the question as to whether similar mechanisms control pigmentation in different species. Our insights into the biological control of pigmentation have principally derived from the study of a few model systems, including salamanders, mice and zebrafish, while theoretical models have focused mainly on mammalian, fish and butterfly pigmentation. In this section we begin by comparing and contrasting pigmentation in these systems, examples of which are shown in Figures 1 and 13, with detailed consideration of the patterning process observed in angelfish and zebrafish. We consider the various theories expounded for the control of pigment regulation, with particular emphasis on the reaction-diffusion based model for pigment patterning.

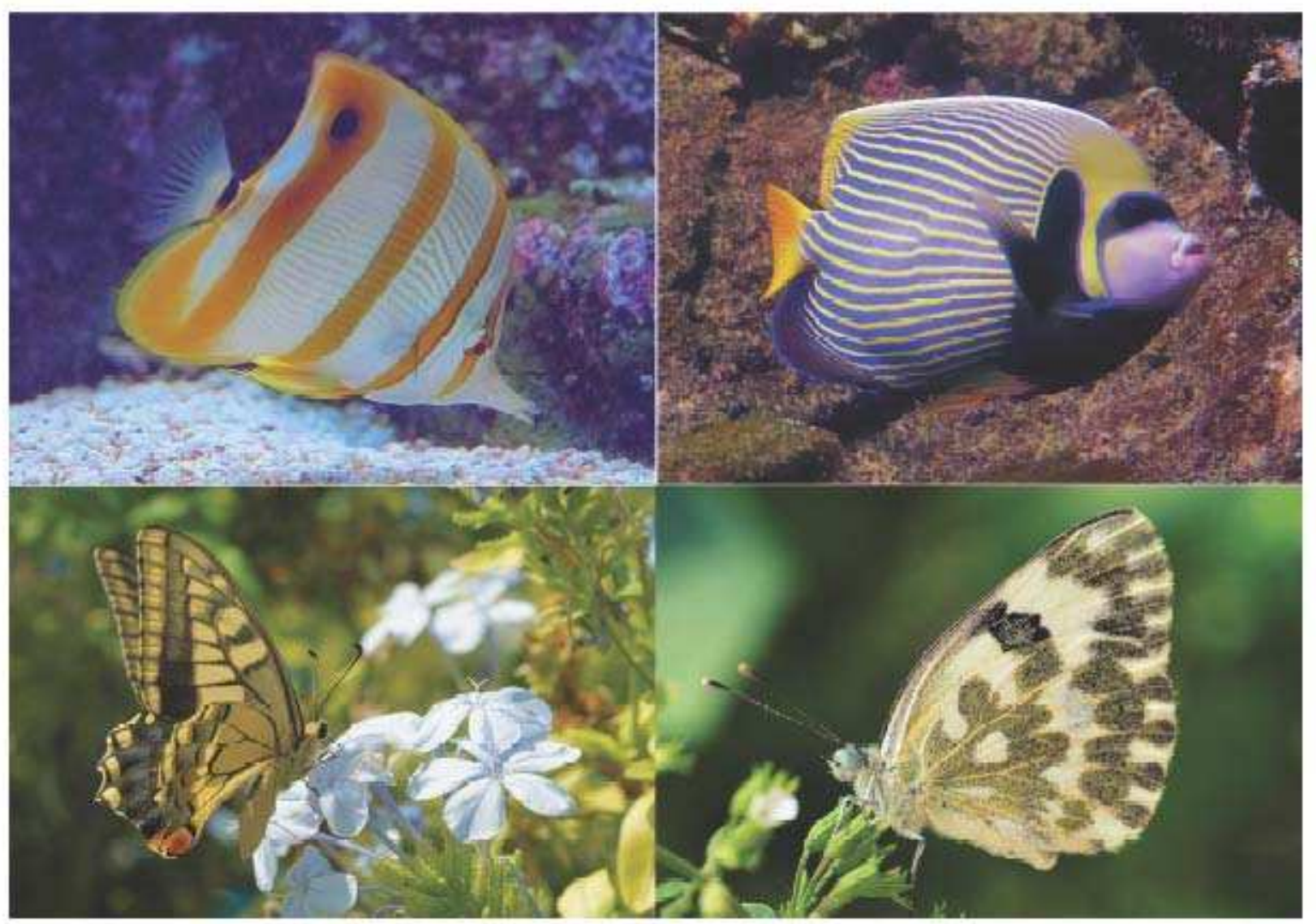

Figure 13: Examples of pigmentation patterns in fish and butterflies. Clockwise from top right: Emperor angelfish (Pomacanthus imperator), Eastern bath white (Pontia edusa), Swallowtail (Papilao machaon) and Beaked coralfish (Chelmon rostratus). Images by K.J. Painter. 


\subsection{Biology of pigmentation}

\subsubsection{Pigmentation in mammals}

In mammals, pigmentation derives from a single pigment cell type - melanocytes - which generate oranges and blacks through transfer of pigment granules via dendrites into hairs and epithelial cells; other colors, such as the blues on the face of a Mandrill, are attributed to the scattering of light within the skin rather than a specific pigment [186]. As noted earlier, pigmentation in mammals and other vertebrates involves the migration of pigment cell precursors from their neural crest origin to the dermal skin layers [119]. Successful migration along a complex path leading to their final destination depends on their ability to respond to local cues (e.g. see [217]), but longrange cues (e.g. chemotactic signals) released in the developing dermis also appear to play a role [216].

It is generally believed that the pigmentation patterns of mammals are fixed early in embryonic development (for example, from the 3rd to 5th week of gestation in the zebra according to [12]) with subsequent alterations stemming mainly from deformation due to organism growth. However, there are a number of notable exceptions, which indicate that the underlying pattern may undergo continual refinement well into juvenile stages. Newborn Malaysian tapirs display an elegant pattern of dappled white stripes and spots on a brown coat which disappear during their first few months into the solid black/white pattern of the adult, while the white spots of foals disappear into an almost uniform brown pigmentation in adult deer. Furthermore, it has been suggested that cat pigmentation markings derive from the same fundamental pattern of "solid spots", which then transform into a wide range of adult patterns (e.g. "rosettes" in leopards and jaguars, spots in cheetahs, "patches" in clouded leopards) during subsequent growth [233]. The evolution of this transition between solid spots and "rosettes" can be observed in leopard and jaguar cubs [122].

\subsubsection{Pigmentation in fish}

Fish (and other animal classes) possess a greater range of pigment cell types (or chromatophores), including melanophores, iridophores and xanthophores, which arrange into ordered layers of the dermis to form the dermal chromatophore unit [9]. The distinct arrangement of these layers generates dazzling and contrasting colors, many of which can not be obtained through a single pigment cell type.

At a macroscopic level, many species of fish (as well as amphibians and reptiles) alter their pigmentation throughout their life cycle. Rapid changes - physiological color change - are stimulated through hormonal and/or neural signals which trigger the redistribution of the pigment granules within the pigment cells, thus allowing animals such as chameleons and flatfish to blend into the surrounding environment. Certain fish can even adopt the form of a chessboard when placed on an appropriate surface [152]. Slower changes - morphological color changes - occur much more gradually and result from changes to pigment cell densities or the transfer of pigment granules to the skin (for a review, see [215]). For example, long-term exposure of the medaka (Oryzias) to a dark background results in increased densities of melanophores and decreased numbers of leucophores [214]. In many instances, the pigmentation pattern is dynamic, undergoing continuous 
transformation as the fish develops from larval to adult stages. Consequently, fish pigmentation offers a tractable system for studying the intrinsic relationship between embryonic growth and morphogenetic patterning. Two examples have attracted particular attention from theoreticians: the marine angelfish and the zebrafish.

\section{Pigmentation in the marine angelfish}

The marine angelfish family Pomacanthidae comprises 9 genera and more than 70 individual species found in tropical waters of the Atlantic, Indian and Pacific oceans [153], with their exquisite and diverse coloration popularizing them as ornamental fish for aquariums. One of the striking characteristics for many of the species in this family are the markedly different juvenile and adult markings. Most species in the genus Pomacanthus (and sub-genus Pomacanthus Euxiphipops) display a broadly consistent juvenile pattern composed of a number of curved white bars on a dark blue background. During juvenile growth, the numbers of these stripes increases in proportion to fish size with new stripes regularly inserting between pre-existing ones [113], first emerging faint and narrow but widening as the fish continues to grow. This process may repeat several times before being replaced by the adult pattern, which varies widely from species to species (e.g. see [2]). Adoption of a distinct pattern confers an advantage to the juvenile: adults are highly territorial and tend to attack other fish with similar markings [74].

\section{Pigmentation in zebrafish}

The beautiful coupling between organism growth and pattern formation described above is offset by its impracticality for detailed study: the long period of growth, large size, sensitivity to the environment and territorial nature preclude intensive study of Pomacanthus in the laboratory. In stark contrast, the zebrafish Danio rerio has emerged as one of the predominant model organisms for studying embryonic development (for a review, see [84]). Unsurprisingly, the pigmentation pattern is one of its defining traits, consisting of alternating black and silver/yellow stripes that extend horizontally across the flank.

Zebrafish pigmentation appears early and melanophores are already visually identifiable 24 hours post fertilization (hpf) and the pattern is dynamic from early larval through to adult stages. The rapid development of zebrafish (3-4 weeks from fertilization to adult), the large repertoire of larval and adult pigmentation mutants $[107,159,85]$, the number of closely related species with varied markings [175] and the growing understanding of the molecular events controlling pigment cell differentiation and positioning [208] collectively make zebrafish pigmentation an ideal system for studies of morphogenetic patterning.

Pigmentation can be divided into two distinct phases: a larval pattern that begins to form 24 hpf, and the characteristic horizontal black/silver stripe pattern of the adult. Pigment cell precursors originate in the neural crest (a transitory population of precursor cells also giving rise to neural, glial and skeletal cells) along the dorsal midline between the neural tube and surface ectoderm [119]. Melanin-expressing melanophores can be identified shortly following their migration away from the crest where they begin to populate the dorsal stripe, one of three melanophore stripes that characterizes the larval pattern [136]. Patterning of the dorsal stripe initiates anteriorly 
and progresses posteriorly, consistent with the migration of cells away from the neural crest. After $30 \mathrm{hpf}$ melanophores begin to form a ventral stripe with a lateral stripe the last to become established (at approximately $42 \mathrm{hpf}$ ). Two further pigment cell types contribute to the larval pattern: xanthophores (yellow) in melanophore-free zones and iridophores associated with melanophores in the stripes [91]. Over subsequent days of development this basic pattern is consolidated through increased numbers of pigment cells.

Metamorphosis to the adult pattern commences two to three weeks post-hatching. Melanophores begin to appear from latent precursors [179] to form the first two adult stripes on either side of the larval lateral stripe. An interstripe predominantly composed of xanthophores develops in between, while those melanophores in the larval lateral stripe either die or migrate into one of the forming stripes [178]. The formation of adult stripes and interstripes appears to partially depend on the attractive and repulsive relationships between the various chromatophore types $[125,177]$. During the following days, pigment cell numbers increase to reinforce the stripes and interstripes until each stripe is densely populated by pigment cells and approximately 6 cell diameters in width. With continued adult growth, new stripes/interstripes appear dorsally and ventrally of the existing adult stripes [130], although occasionally an existing adult stripe may split (particularly in older fish, [6]). For further details on metamorphosis to the adult pattern see, e.g. [99, 176, 125, 175].

\subsubsection{Pigmentation in butterfly wings}

Pigmentation patterns in butterfly wings form in a flat two-dimensional structure composed of a highly organized mosaic of individually colored "tiles": the pigmented scales [156]. A subset of epidermal cells differentiates to form a monolayer of scale cells that subsequently extend broad and flat cellular appendages, the scales. Coloration of the scale is achieved through the transfer of pigment granules synthesized in the scale cell, each of which can produce a single pigment color, [111]. Synthesis of the pigment, and hence appearance of the color pattern, does not occur until shortly before the emergence of the adult butterfly (i.e. late pupal stages), yet specification of the color type of scale cells is established during larval and early pupal stages, thereby generating an imperceptible prepattern of scale cells each committed to a pigment type. The fixed structure of the scale cell monolayer precludes cell movement during the specification of the scale cells, which excludes the involvement of movement during pattern organization [156]. This is in stark contrast to vertebrates where pigment cells can potentially migrate within the dermis.

Each of the attractive and complex pigmentation markings observed in the wings of different species can be broken down into common sets of elemental pattern types, referred to as the nymphalid groundplan [196, 213, 156, 160]. Patterning observed on wing patterns is comprised of both "localized" forms, such as the characteristic "eyespots", an example of which can be seen to the bottom left of the Swallowtail wing pattern in Figure 13, or the specific markings correlated with structural elements of the wing (e.g. the thin black markings along the wing veins of the Swallowtail) and "global" forms consisting of the larger scale patterns spanning entire wing tracts, for example the alternating dark/white patchwork pattern on the Eastern Bath White (Figure 13) or the wide black band running parallel to the distal wing in the Swallowtail.

At a cell and molecular level, the determination of eyespots (in Nymphalidae) is currently the 
most well understood process. Eyespots usually consist of a pattern of concentric color rings used as a means of startling predators. Each eyespot develops about a group of organizing cells that act as signaling centers, and while the signal is not known, the fact that the cells signal is demonstrated by transplanting them to other wing regions and observing the formation of ectopic eyespots at the new locations [30]. In [37] the expression patterns of a number of candidate genes was explored and the eyespot organizing cells at the center were identified by their unique expression of Distalless. A variety of further transcription factors have been found to be expressed in rings coincident with the color markings [32]. These results imply a model in which organizer cells stimulate gene expression and, eventually, pigment specification in the surrounding cells.

\subsection{Chemical prepattern and reaction-diffusion models for pigmentation}

\subsubsection{Mammalian pigmentation}

Much of the current interest in modeling pigmentation patterning stems from the influential work of Murray on mammalian coat markings and lepidopteran wing patterns in the late 1970s (see [143, 144, 145]; a comprehensive and detailed review of this topic is given in Chapter 3 of [146]). Murray's proposal was that a system of reacting and diffusing morphogens could generate a chemical pre-pattern within the developing integument via a Turing instability. The subsequent coat pattern was simply assumed to reflect the chemical pre-pattern via differential response of the pigment cell precursors to the levels of these morphogens. A similar reaction-diffusion based model for mammalian pigmentation was developed in [13].

One of the compelling arguments for the reaction-diffusion theory of pigmentation lies in its impressive capacity to replicate many of the observed pigment patterns through a single mechanism. We consider a two component Turing model given by Equation (2.3), with $c=(u, v)$ representing hypothetical chemical morphogens in the integument. Assuming that (a) the depth of the integument is small with respect to the scale of patterns, and (b) the initial pigmentation pattern is fixed over a relatively short timescale early in development, it is reasonable to assume the reaction takes place on a fixed 2D domain (we consider coupling growth to pigmentation patterning later). For the simulations here the reaction term $\bar{R}$ is given by the Lengyel-Epstein model [120] developed to describe the Turing patterns observed in the CIMA chemical reaction [39], although many other kinetics would generate equivalent results. The equations are given by

$$
\begin{aligned}
& \frac{\partial u}{\partial t}=D_{u} \nabla^{2} u+k_{1}\left(v-\frac{u v}{1+v^{2}}\right) \\
& \frac{\partial v}{\partial t}=D_{v} \nabla^{2} v+k_{2}-v-\frac{4 u v}{1+v^{2}}
\end{aligned}
$$

on a $2 \mathrm{D}$ rectangular domain $\Omega$ with periodic boundary conditions. A stability analysis about the positive homogeneous steady state at $\left(1+k_{2}^{2} / 25, k_{2} / 5\right)$ determines the conditions on the parameters for Turing instabilities to occur and in Figure 14 we plot the spatial distributions of activator ( $v$ ) predicted by equations (5.1)-(5.2) at various points in $D_{v}-k_{1}$ space. Clearly, the above system is capable of producing many of the commonly occurring pattern-types observed in animal skins, 
including "spots", "stripes", "reticulated stripes" and "inverted spots". The conditions that distinguish between stripes or spots in reaction-diffusion systems have been investigated by a number of authors; a major factor is the dominating nonlinearity in the kinetics (e.g [124, 66, 132]).

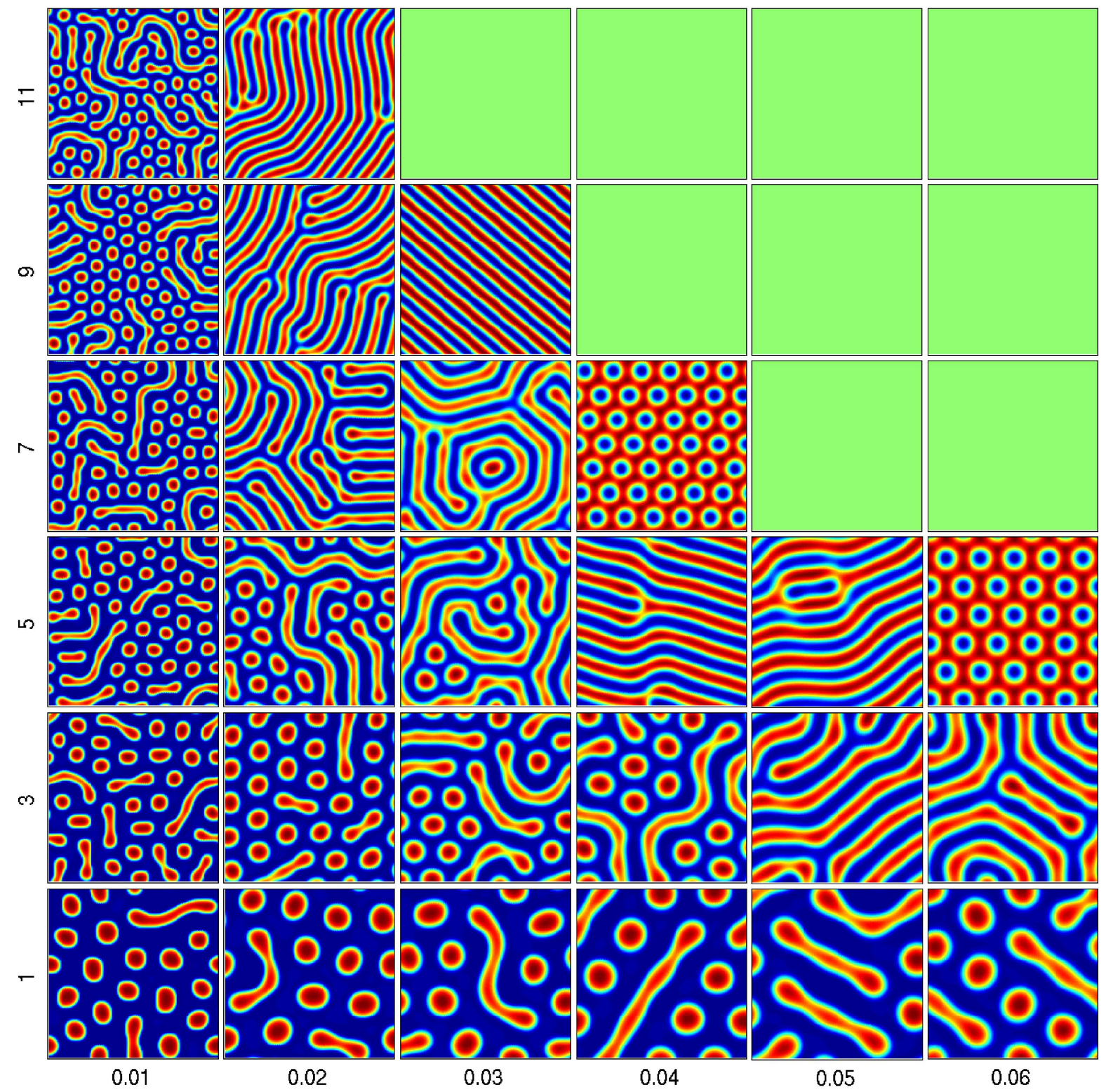

Figure 14: Activator $(v)$ concentrations at different values of $D_{v}$ (horizontal axis) and $k_{1}$ (vertical axis). At each $D_{v}-k_{1}$ pair, equations (5.1)-(5.2) were solved numerically to $t=10000$ from initial distributions $(u(\underline{x}, 0), v(\underline{x}, 0))=\left(u_{s}, v_{s}\right)+r(\underline{x})$, where $\left(u_{s}, v_{s}\right)$ is the uniform steady state and $r(\underline{x})$ a small random spatial perturbation. Other model parameters were fixed at $D_{u}=1.0$ and $k_{2}=11.0$ with periodic boundary conditions on the 2D square domain of dimensions $10 \times 10$. Concentration is represented through the colorscale $(\mathrm{red}=$ high, blue $=$ low). Note that in the top right hand corner, the conditions for a Turing instability are not satisfied and no spatial pattern develops. 
Predictions of the reaction-diffusion model stem from the link between geometry and patterning: the patterns generated by Turing systems possess a characteristic wavelength (see Figure 14) and it is therefore expected that average widths/diameters of stripes/spots are broadly consistent across the body. The intrinsic relationship between the pattern and the domain geometry subsequently leads to a number of generic predictions. For example, spotted mammals can exhibit striped tails while the reverse does not occur, and both very small and very large mammals can generally be expected to display uniform pigmentation (see [146] for further details). The former prediction appears to hold true while the latter, albeit not universal (giraffes and species of vole provide counter-examples at either end of the size distribution), generally seems to be upheld. At a finer scale, reaction-diffusion models have also been proposed to provide the information for the positioning of the individual hair follicles $[150,151,140]$ and recent experimental data have revealed potential networks that may operate in a Turing-like fashion ([142, 207], see also [87] this issue).

While the reaction-diffusion model is far from the only hypothesis for the origin of pigmentation patterns, see Section 5.3.), the seductive charm lies in its capacity to generate highly realistic patterns (particularly when solved on life-like geometries, see Figure 15) and has led to its application to model pigment pattern control across the animal kingdom. Reaction-diffusion models have been employed in [199] to explain butterfly wing markings (see section 5.2.3. below), Meinhardt [133] uses activator-inhibitor systems to replicate the enormous diversity of patterns of tropical sea-shells, their application to the markings of various species of ladybird beetles is considered in [121], and [187] have examined within-feather pigmentation control via a Turing system. Over the past decade interest in reaction-diffusion models has been further heightened by their ability to replicate the highly dynamic patterns observed in the skins of fishes.

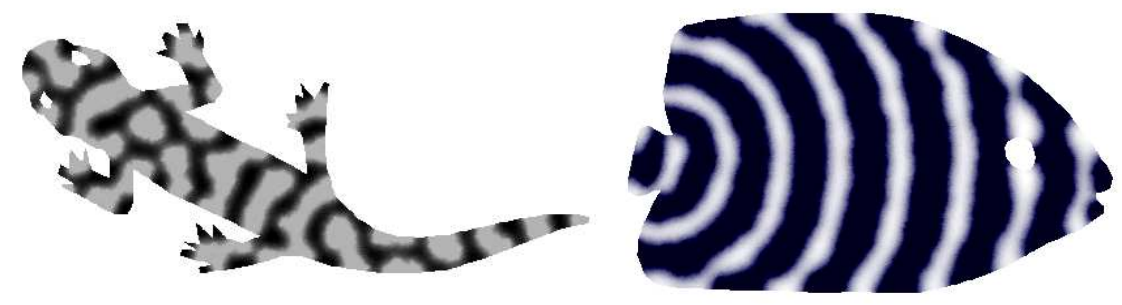

Figure 15: Simulations of equations (5.1)-(5.2) on "animal-shaped" geometries replicating the pigmentation patterns in Tiger Salamander (Ambystoma tigrinum) (left) and Juvenile Koran Angelfish (Pomacanthus semicirculatus) (right).

\subsubsection{Fish pigmentation}

The first proposal for a reaction-diffusion model controlling fish pigmentation was formulated by Kondo and Asai [113] following observations on the patterning of the marine angelfish genus Pomacanthus. As previously described, juvenile members display evolving patterns in which the number of stripes increases proportional to body length before metamorphosis to the adult pattern 
occurs. The close correspondence between simulations of reaction-diffusion models and temporal features of the pattern change observed in real fish led to the proposal for a reaction-diffusion model driving angelfish pigmentation [113] (see Section 5.4.). A number of further reaction-diffusion based models have been developed to describe additional features of pigmentation in Pomacanthus and other species: Barrio and others $[225,15]$ have considered the special role that domain geometry and boundary conditions may play; Painter and others [173, 172] augmented a reactiondiffusion model to incorporate the accumulation of pigment cells into stripes via chemotaxis. A number of authors have explored the mechanisms regulating stripe orientation, (eg., [204, 205] and [135]). The latter demonstrated how stripes of specific orientations can be experimentally generated in the CIMA chemical reaction.

A reaction-diffusion model for adult zebrafish patterning has been proposed in [6], indicating that perturbation to a single parameter in a standard reaction-diffusion system could generate a transition between horizontal stripes (as observed in the zebrafish Danio rerio) to labyrinthine and spotted patterns (observed in the leopard mutant). In later work by the same group, experimental results in which zebrafish stripes reformed following their partial ablation were replicated using a reaction-diffusion model, which suggests that the adult stripe pattern is dynamically-controlled rather than fixed at its time of formation [242].

\subsubsection{Butterfly pigmentation}

As described above, the complexity observed in butterfly wing pattern can be broken down into a number of distinct pattern elements [196, 213, 156], and theoretical modeling has generally concentrated on understanding the control of these various elements separately. The mechanism for eyespot formation has been considered by a number of authors. Nijhout [155] proposed that cells at the eyespot focus could produce a signal, leading via diffusion to a morphogen gradient that provides positional information to the surrounding cells. A number of detailed investigations have explored these ideas in greater detail (e.g. [156, 139, 55]). In contrast, the models in [67, 129], are aimed at explaining eyespot generation through simulating candidate gene regulatory networks. The pre-specification of eyespot organizers from an undifferentiated population of scale cells has been modeled in [156] using an activator-inhibitor mechanism. This model was capable of producing point-wise chemical prepatterns at the precise locations of future organizers by assuming wing veins act as a source of activator. Furthermore, chemical concentrations predicted in the model displayed a complex temporal sequence later shown within the gene-expression patterns of Distal-less.

A number of models have been developed to describe the mechanistic basis for global patterning features, i.e. patterning that covers large portions of the wings (see [157] for a detailed review). Murray [145] proposed a diffusion-morphogen-gene-activation model in which wing margins provide the source for a diffusible morphogen which, in turn, triggers gene activation and eventual color specification. Theoretical results of this model were consistent with a wide variety of observed patterns. A diffusion-based model has been developed by Bard and French in [14]. Eyespots and the wing-margin are assumed to act as sources and sinks for a chemical morphogen, the subsequent profile of which is assumed to provide the positional information for color specifica- 
tion. More recently, work by Sekimura and others (e.g. [199, 126, 157]) has explored the potential for reaction-diffusion type mechanisms to generate features of global patterning. Focusing on the spectacular and varied color patterns of the species Papilio dardanus, a standard activator-inhibitor type mechanism has been solved on realistic wing geometries with specific boundary conditions (representing activator sources) along the distal wing edges. Perturbations to model parameters were shown to generate the variety of patterns observed in this species.

\subsection{Cell-based models for pigmentation}

Despite the successes of the reaction-diffusion model at capturing certain patterning elements across the animal kingdom, including mammalian, fish and butterfly pigmentation, the in vivo identification of plausible morphogens is lacking and the model does not consider the potentially important role the pigment cells themselves may play in setting or regulating the pattern (e.g. $[177,125])$. In the reaction-diffusion model it is generally assumed pigment cells are "passive" objects that respond to threshold concentrations of the morphogens through differentiation, but a number of other models have been developed that explicitly incorporate a role for cells in the patterning process.

In vertebrates, pigment cells derive from the neural crest, an embryonic population contributing to a variety of cell populations in the adult organism. During development neural crest cells are highly motile, occasionally traveling across the organism to their final destination, and it is thought that both local and long distance chemical signals control neural crest cell migration in vivo. Based on data collected on the timing of stripe formation in embryonic and hatchling alligators, [147] proposed a cell-chemotaxis model for pigmentation, while [148] suggested a similar model for pigmentation in snakes. In these models, the pigment cells play a crucial role in generating the pigment pattern through migration in response to self-regulated morphogens. As described in the context of bacterial patterning, models of this form can certainly generate macroscopic patterns qualitatively similar to observed pigment patterns. Chemotaxis has also been incorporated into the models by Painter et al. [173, 172] to explain specific aspects regarding the dynamic pigmentation patterns in the angelfish genus Pomacanthus. In these models the cells respond chemotactically to the primary pattern generated by a Turing system, rather than inducing their self-organization.

Based on the findings in [177, 125], recent discrete-cell models developed in [141] and [36] dispense with extrinsic chemical signals and demonstrate that the pigmentation pattern for zebrafish can be generated solely through specific local interactions (i.e. adhesive or repulsive) between the principal pigment cell types (melanophores and xanthophores). Perturbations to the self- (i.e. melanophore-melanophore or xanthophore-xanthophore) and cross- (melanophore-xanthophore) interactions generates a wide variety of pattern types corresponding to pigmentation mutants. The capacity for differential-adhesion to generate the cellular sorting of different populations has been known since the seminal work of Steinberg and others (e.g. see [212]).

Other discrete cell models for pigmentation have been developed in [243] and [79] for mammalian pigmentation, adopting cellular-automata type models. While pigment cells are explicitly incorporated into these models, their input into the patterning process occurs via their regulation of chemical activators and inhibitors, subsequently leading to similar pattern phenotypes to those 
generated by continuous reaction-diffusion systems.

\subsection{Patterning on growing skins}

In the foregoing we have generally restricted attention to the modeling of pigmentation patterning on fixed geometries. Yet, for many species, the color markings undergo continual change throughout the lifespan of the organism, a striking example of which is given by the juvenile angelfish described earlier [113]. Adult zebrafish also show stripe insertion, with new stripes inserted dorsally and ventrally [130] following sufficient adult growth. As previously mentioned, there is also evidence of transformations during mammalian pigmentation, particularly in certain species of cats $[233,122]$.

These examples all imply a link between morphogenetic patterning and domain growth: the insertion of new stripes in juvenile angelfish is closely correlated with fish size, with stripes increasing twofold with each doubling in the head-tail length. There is also an age-structure to the stripes, with old stripes bright and wide and newer stripes faint and thin. To explain these transformations it is necessary to account for the domain growth, as has been done by a number of authors (e.g. [5, 114, 113, 173, 49, 57, 48, 11]). In general, tissue growth will depend on a range of both global and local cues (for example mitotic and apoptotic factors) and expansion is expected to depend on the spatial distribution of underlying factors transported with tissue growth and models for "morphogen" controlled domain growth have been developed by [57, 49, 48, 11]. Earlier (see section 2.6.) we developed a model to describe the planar expansion of a tissue through cellular growth, and demonstrated that under the assumption of spatially uniform growth the equations simplify to a form that lacks the additional terms resulting from growth-induced transport.

\subsubsection{Stripe insertion in juvenile angelfish: uniform growth}

To model stripe insertion in juvenile angelfish it is necessary to consider the in vivo growth of juveniles. In the absence of empirical data it is reasonable to expect uniform growth and equations (5.1)-(5.2) are extended onto a $1 \mathrm{D}$ growing domain $[0, L(t)]$ representing the head-tail axis. Following the process in Section 2.6. and rescaling onto a fixed domain $[0, L(0)]$, the equations for a growing-domain are given by

$$
\begin{aligned}
\frac{\partial u}{\partial t} & =\frac{D_{u}}{L(t)^{2}} \frac{\partial^{2} u}{\partial x^{2}}+k_{1}\left(v-\frac{u v}{1+v^{2}}\right)-\frac{L^{\prime}(t)}{L(t)} u \\
\frac{\partial v}{\partial t} & =\frac{D_{v}}{L(t)^{2}} \frac{\partial^{2} v}{\partial x^{2}}+k_{2}-v-\frac{4 u v}{1+v^{2}}-\frac{L^{\prime}(t)}{L(t)} v
\end{aligned}
$$

The impact of growth is absorbed into time-dependent diffusion coefficients and additional "decaytype" terms interpreted as chemical dilution due to domain growth. Analogous equations have been derived by [114], [173] and [49] using a variety of methods. In the context of angelfish pigmentation, the principal question is whether the above generates a regular insertion of new stripes as the fish doubles in size. To explore this we simulate equations (5.3)-(5.4), assuming that juvenile growth (at least initially) is exponential (the impact of different growth forms has 

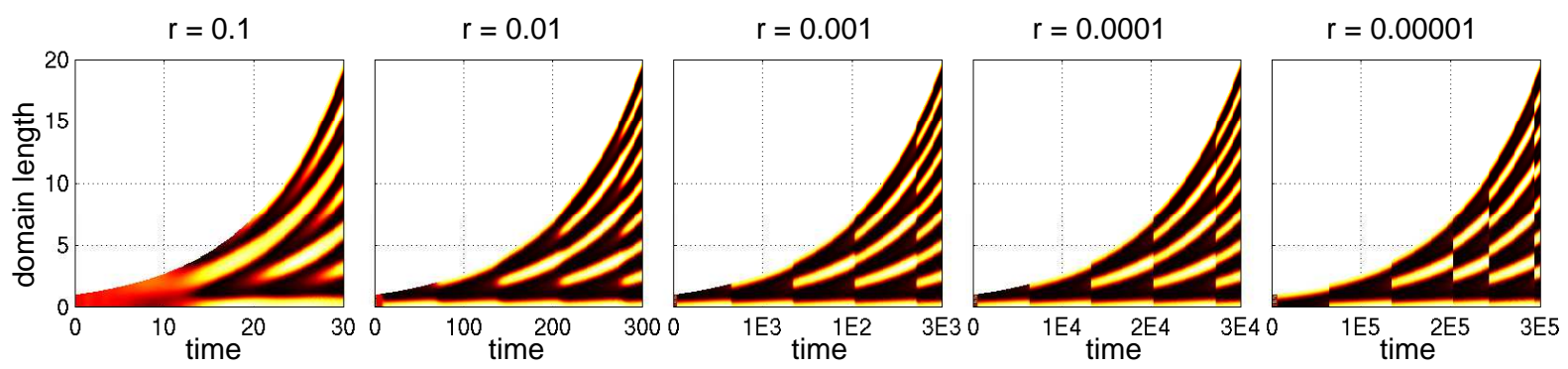

Figure 16: Simulations showing activator $(v)$ concentration from equations (5.3)-(5.4) for a 1D exponentially growing domain $[0, \exp (r t)]$. Simulations show that for a wide range of growth rates it is possible to generate a robust sequence of "frequency doubling" in which new activator peaks insert between preexisting ones. For very slow and very fast growth rates, this sequence breaks down.

been considered in [173, 49]), thus $L(t)=L_{0} \exp (r t)$ where $r$ is the growth rate. The activator distribution $(v)$ is shown in Figure 16, and the results demonstrate that for a wide range of growth rates the reaction-diffusion model does indeed generate a robust "doubling" sequence in which activator peaks insert with each doubling of the domain length, consistent with stripe insertion on the angelfish.

That a Turing system could generate "peak doubling" was predicted in [5] and re-discovered in the context of juvenile angelfish patterning by Kondo and Asai [113], strengthening their hypothesis for an underlying reaction-diffusion system governing pigmentation. A number of subsequent extensions of their analysis have been made to understand additional features in the pigmentation sequence. For example, Painter et al. [173, 172] analyzed patterning on more realistic two-dimensional geometries and incorporated a chemotaxis-based hypothesis for the accumulation of pigment cells into the forming stripes. On the 2D uniformly growing domain $(x, y) \in\left[0, L_{1}(t)\right] \times\left[0, L_{2}(t)\right]$, their equations are

$$
\begin{aligned}
& \frac{\partial c}{\partial t}=\frac{1}{L_{1}^{2}} \frac{\partial}{\partial x}\left(D_{c} \frac{\partial c}{\partial x}-\chi_{c} c \frac{\partial u}{\partial x}\right)+\frac{1}{L_{2}^{2}} \frac{\partial}{\partial y}\left(D_{c} \frac{\partial c}{\partial y}-\chi_{c} c \frac{\partial u}{\partial y}\right) \\
& \frac{\partial u}{\partial t}=\frac{D_{u}}{L_{1}(t)^{2}} \frac{\partial^{2} u}{\partial x^{2}}+\frac{D_{u}}{L_{2}(t)^{2}} \frac{\partial^{2} u}{\partial y^{2}}+k_{1}\left(v-\frac{u v}{1+v^{2}}\right)-\left(L_{1}^{\prime}(t) / L_{1}(t)+L_{2}^{\prime}(t) / L_{2}(t)\right) u,(5.6) \\
& \frac{\partial v}{\partial t}=\frac{D_{v}}{L_{1}(t)^{2}} \frac{\partial^{2} v}{\partial x^{2}}+\frac{D_{v}}{L_{2}(t)^{2}} \frac{\partial^{2} v}{\partial y^{2}}+k_{2}-v-\frac{4 u v}{1+v^{2}}-\left(L_{1}^{\prime}(t) / L_{1}(t)+L_{2}^{\prime}(t) / L_{2}(t)\right) v
\end{aligned}
$$

where the additional equation for $c$ represents the density of the predominant pigment cell type populating the "stripes". In Figure 17 we show the results of simulations of the above model using "logistic" growth, for which the initial growth is approximately exponential, but later the domain size stabilizes. The activator concentration $(v)$ is plotted in the top row, showing that a robust sequence of stripe insertion also occurs in 2D, but it should be noted that the developing stripes show no persistent age structure. However, the corresponding pigment cell density (bottom row) does show an age structure to the stripes due to the slower process of chemotactic migration. 




Figure 17: Simulation of the 2D chemotactic-reaction-diffusion hybrid model showing (top row) activator concentration $v$ and (bottom row) pigment cell density $c$. The accumulation of cells at the sites of "new" stripes proceeds slowly, resulting in an age-structure to the stripes consistent with the pattern observed in juvenile angelfish.

\subsubsection{Stripe insertion in zebrafish: boundary growth}

While new stripes appear throughout adult zebrafish growth, their pattern of appearance is somewhat different from that of juvenile angelfish: new zebrafish stripes appear along the dorsal and ventral edges of the flank with increased growth. Clearly, the uniform growth scenario described above is unable to explain this sequence, since uniform growth predicts the appearance of new stripes across the entire dorsal-ventral flank. We consider the possibility that growth in adult zebrafish occurs principally through stretching at the dorsal/boundary extremes, i.e. boundary growth. For example, by assuming domain growth occurs along the $y=0$ boundary (representing the dorsal flank), the reaction-diffusion systems equations become [172, 49]

$$
\begin{aligned}
& \frac{\partial u}{\partial t}=\frac{D_{u}}{L_{x}(t)^{2}} \frac{\partial^{2} u}{\partial x^{2}}+\frac{D_{u}}{L_{y}(t)^{2}} \frac{\partial^{2} u}{\partial y^{2}}+\frac{y L_{y}^{\prime}}{L_{y}} \frac{\partial u}{\partial y}+k_{1}\left(v-\frac{u v}{1+v^{2}}\right) \\
& \frac{\partial v}{\partial t}=\frac{D_{v}}{L_{x}(t)^{2}} \frac{\partial^{2} v}{\partial x^{2}}+\frac{D_{v}}{L_{y}(t)^{2}} \frac{\partial^{2} v}{\partial y^{2}}+\frac{y L_{y}^{\prime}}{L_{y}} \frac{\partial v}{\partial y}+k_{2}-v-\frac{4 u v}{1+v^{2}} .
\end{aligned}
$$

The nonuniform growth results in the addition of convective terms describing the transport of the chemicals due to the stretching of the skin, as in [57]. Under reasonable forms for the growth terms, the above equations can indeed predict the appearance of new stripes along the dorsal extremes, consistent with the mode of stripe insertion in zebrafish, as shown in Figure 18. 
Domain growth

- Position of new stripe
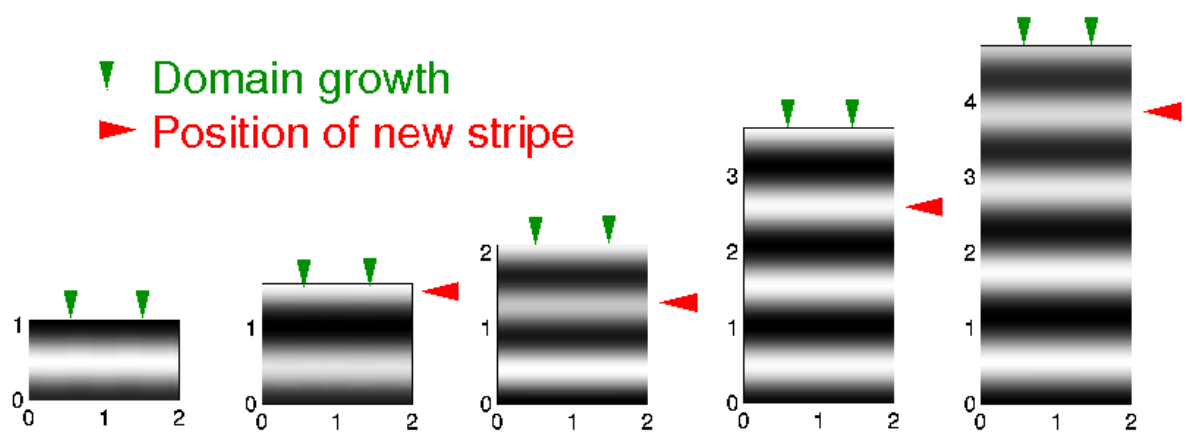

Figure 18: Simulation of the 2D boundary growth model demonstrating (top row) insertion of new stripes along the dorsal side. For these simulations, we assumed $L_{x}(t)=2$ and $L_{y}(t)=1+r t$ for $r=0.01$.

\section{Future Perspectives}

Recent years have witnessed a dramatic rise in the use of mathematical modelling to understand the basic mechanistic processes underlying biological pattern formation, from patterning within an individual cell, via the organization of tissues and organs during development to the coordinated behaviour of organisms within a population. The advancement in experimental methodology is leading to increasingly detailed and quantitative data on the core regulators of pattern formation, in turn resulting in a new generation of detailed mathematical models both driven by and driving experimental research. However, more traditional, phenomenological-based models still play a vital role, providing an established theoretical framework in which to understand details of these more complex models. We conclude this paper with a brief consideration of outstanding questions relating to pattern formation both in general and with respect to the three examples reviewed earlier - Drosophila, E. coli and pigmentation patterning - highlighting the core role that modelling may play in this process.

\subsection{Drosophila}

The timing is propitious for mathematical modeling of pattern formation and morphogenesis in Drosophila, as there is a good balance of established facts and questions that remain to be answered. In recent years, a number of biological questions relied on developing and analyzing mathematical models of varying complexity, as discussed earlier, but there is ample scope for further work. As discussed for each case earlier, to truly couple the modeling with the experimentation to make the next wave of biological discoveries, one has to develop more geometrically representative models of the organisms and tissues used for the experimentation. While significant 
biological insight has been gained from simple 1D models, we have reached the limits of what we can use the models for in terms of leading experimental design. Furthermore, experimentalists have been exploiting the orthogonal nature of AP and DV patterning to drive ectopic expression of DV genes in spatially restricted zones along the AP axis. These types of experiments provide more flexibility in developing experiments to delineate the function of BMP regulators, and none of the current 1D models are capable of predicting the signaling patterns for any of these experiments. Furthermore, experimentalists used a method of mRNA injection to decipher various aspects of BMP-mediated positive feedback in the Drosophila embryo. In order to test the next generation of models and plausible positive feedback mechanisms against the experimental observations, a full 3D model must be developed and this work is ongoing.

With a 3D organism-scale model of the Drosophila embryo, modelers can investigate a number of other phenomena that have not yet been addressed. First, one could perturb the geometry of the model to determine the sensitivity of patterning to the specific embryo geometry. One could also couple the 3D model with new biological data such as the VirtualEmbryo, which is a new spatiotemporal map of gene expression in Drosophila, and determine the sensitivity of BMP signaling to perturbations in the spatial distribution of upstream components.
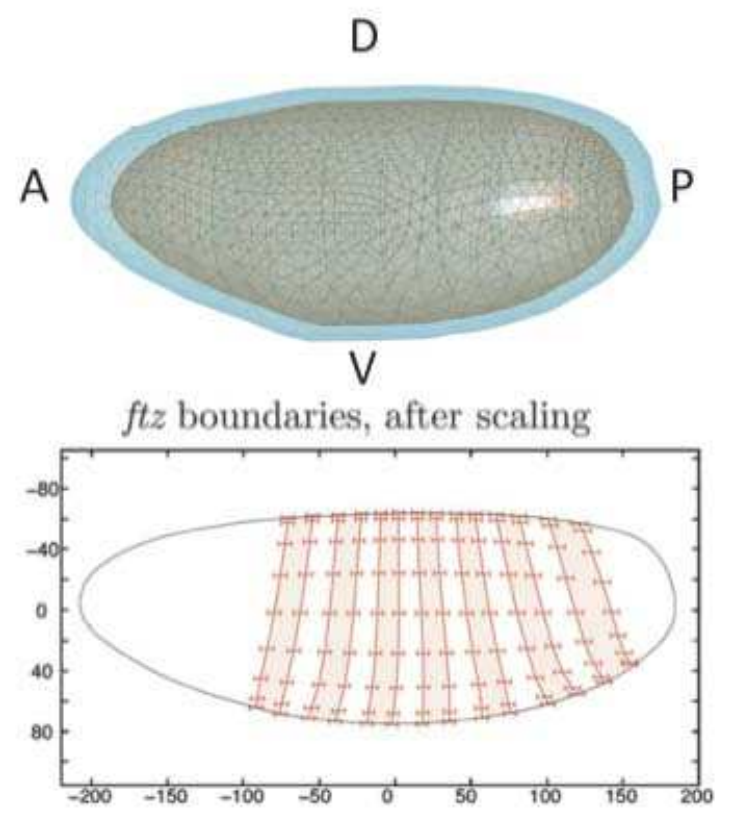

Figure 19: Future work- geometry, dynamics and concentration data. (From [108] with permission)

\subsection{Bacteria}

Biofilm formation is a complicated process that involves cell growth, differentiation, cell-cell signaling, and mechanical response to substrate. Bacterial patterns formed in laboratories provide a 
manipulable model system to understand biofilm initiation and growth. In particular, the beautiful patterns formed in E. coli colonies have posed excellent challenges to understand the complex interplay between different processes involved through mathematical modeling. Although the previous models have been shown to produce qualitatively similar patterns in experiments, the understanding of the mechanisms underlying these patterns is still poor and has to be addressed.

- Further development of the hybrid cell-based model. The hybrid cell-based model provides opportunities for modelers to describe individual cells in great detail and investigate the role of intracellular processes in the populational patterns. Two major aspects of the E. coli patterns in semi-solid agar are the swarm ring formation and the regular aggregation pattern formation. The hybrid cell-based model has already been shown to predict the outwardmoving swarm ring, with a sharper wave front and a diffusive wave tail. Depending on parameters, instability develops inside the ring and may induce fast moving aggregates. In order to obtain stable aggregates disassociated from the ring, one has to incorporate saturation of receptors at high concentration of attractant and change of motility of cells in the future. In addition, since succinate has been shown to be a weak attractant for $E$. coli , it is worthwhile to investigate the contribution of response towards multiple signals to the pattern formation.

- Derivation of continuum models. In section 4.4., chemotaxis equations were derived from the cell-based model under the shallow signal gradient condition. How to derive the macroscopic equations for large signal gradient remains an open problem. In addition, in the derivation cell-cell mechanical interaction was ignored since the cell density is low. However when cell density becomes high, one may need to consider the volume filling effects. How to derive the macroscopic equation with volume filling from a suitable cell-based model deserves future investigation. An initial step to address the question would be to consider the case with no signal gradient and derive a nonlinear diffusion equation.

\subsection{Pigmentation patterns}

Pigmentation patterning presents an ideal system for understanding the emergence of pattern and form in the developing embryo. The rich tradition of modeling in this area provides a convenient platform for formulating expanded models that integrate recent data regarding the molecular controls and cellular interactions determining pigment cell specification and positioning. The natural cell markers (i.e. the pigment) and the ability to follow pattern change in a noninvasive manner offers a potential to formulate testable predictions into the fundamental mechanisms controling patterning. To conclude this section, we offer a number of challenges for modelers to address in this exciting area.

- Distinguishing between models for pigmentation. As described above, the reaction-diffusion model accurately predicts many known pigment patterns across a wide variety of species. Yet a number of diverse models, including chemotaxis-based and differential-adhesion-based models, are capable of predicting similar pattern types. How do we successfully differentiate 
between these various models to identify the fundamental mechanism underlying pigmentation control?

- Integrating microscopic data into the modeling. In the absence of detailed information concerning the cellular and molecular processes underpinning patterning, the majority of modeling has focused on a phenomenological level, employing generic assumptions regarding the underlying interactions. Recent work, particularly in zebrafish and butterflies, is revealing greater insight into molecular and cellular interactions, leading to recent formulations of cell-based models of pigmentation control [141,36]. A challenge for future modeling will be to integrate new data into existing models to generate experimentally testable predictions.

- Positioning of pigment cell precursors. Pigmentation models have generally focused on explaining the origin of the adult form with less attention on the initial stages. Following their migration from the neural crest, larval pigment cells of the zebrafish organise into a number of longitudinal melanophore stripes with xanthophores occupying the spaces in between. The development of models that consider both the generation of this larval pattern as well as its subsequent transformation to the juvenile/adult form remains unexplored.

- Explaining patterning diversity. In zebrafish and related species the pigmentation pattern may vary considerably [175], leading to questions regarding a link between patterning and diversification. A number of theoretical studies have begun to explore the impact of perturbations to a model for the form of the adult pattern (e.g. [6, 141, 36]), yet extending this further will be required with increased understanding into the biological processes governing patterning.

In summary, we are at the beginning of a transition in the modeling of development in biology. Models are changing from qualitative descriptions to explain some peculiar or non-intuitive data, to quantitative models that make predictions that can be tested experimentally to drive the biology forward. As mathematical models of biological mechanisms continue to provide significant new insights into the underlying biology that can be directly assayed in vivo, we will observe a revolution in biology that transforms it from a qualitative, observation-driven discipline, to a quantitative, analysis-driven discipline.

\section{Acknowledgements}

The research herein was supported by the National Science Foundation (US), the National Institutes of Health (US), the Alexander von Humboldt Foundation, the Digital Technology Center, and the Minnesota Supercomputing Institute.

\section{References}

[1] J. Adler. Chemotaxis in bacteria. Science, 153 (1966), 708-716. 
[2] G. Allen, R. Steene, M. Allen. A guide to angelfishes and butterflyfishes. Odyssey, 1998.

[3] K. Amonlirdviman, N. A. Khare, D. R. Tree, W. S. Chen, J. D. Axelrod, C. J. Tomlin. Mathematical modeling of planar cell polarity to understand domineering nonautonomy. Science, 307 (2005), No. 5708, 423-6.

[4] R. P. Araujo, D. L. S. McElwain. A history of the study of solid tumour growth: The contribution of mathematical modelling. Bull. Math. Biol., 66 (2004), No. 5, 1039-1091.

[5] P. Arcuri, J. Murray. Pattern sensitivity to boundary and initial conditions in reactiondiffusion models. J. Math. Biol., 24 (1986), 141-165.

[6] R. Asai, E. Taguchi, Y. Kume, M. Saito, S. Kondo. Zebrafish leopard gene as a component of the putative reaction-diffusion system. Mech Dev, 89 (1999), 87-92.

[7] M. Ashkenazi, H. G. Othmer. Spatial patterns in coupled biochemical oscillators. Jour. Math. Biol., 5 (1978), 305-350.

[8] S. Atkinson, C. Y. Chang, R. E. Sockett, M. Camara, P. Williams. Quorum sensing in yersinia enterocolitica controls swimming and swarming motility. J Bacteriol, 188 (2006), No. 4, 1451-61.

[9] J. Bagnara, M. Hadley. Chromatophores and color change. Prentice-Hall, Eaglewood Cliffs, New Jersey. 1973.

[10] R. E. Baker, E. A. Gaffney, P. K. Maini. Partial differential equations for self-organization in cellular and developmental biology. Nonlinearity, 21 (2008), No. 11, 251-290.

[11] R. E. Baker, P. K. Maini. A mechanism for morphogen-controlled domain growth. J Math Biol, 54 (2007), 597-622.

[12] J. Bard. A unity underlying the different zebra striping patterns. J. Zool., 183 (1977), 527539.

[13] J. Bard. A model for generating aspects of zebra and other mammalian coat patterns. J. Theor. Biol., 93 (1981), 363-385.

[14] J. Bard, V. French. Butterfly wing patterns: how good a determining mechanism is the simple diffusion of a single morphogen? J. Embryol. Exp. Morph., 84 (1984), 255-274.

[15] R. Barrio, C. Varea, J. Aragn, P. Maini. A two-dimensional numerical study of spatial pattern formation in interacting Turing systems. Bull. Math. Biol., 61 (1999), 483-505.

[16] B. L. Bassler. How bacteria talk to each other: regulation of gene expression by quorum sensing. Curr Opin Microbiol, 2 (1999), No. 6, 582-587. 
[17] E. Ben-Jacob, I. Cohen, A. Czirok, T. Vicsek, D. L. Gutnick. Chemomodulation of cellular movement, collective formation of vortices by swarming bacteria, and colonial development. 238 (1997), No. 1, 181-.

[18] E. Ben-Jacob, I. Cohen, H. Levine. Cooperative self-organization of microorganisms. Advances in Physics, 49 (2000), No. 4, 395-554.

[19] E. Ben-Jacob, I. Cohen, O. Shochet, I. Aranson, H. Levine. Complex bacterial patterns. 373 (1995), No. 6515, 566-557.

[20] E. Ben-Jacob, O. Schochet, A. Tenenbaum, I. Cohen, A. Czirok. Generic modelling of cooperative growth patterns in bacterial colonies. 368 (1994), No. 6466, 46-49.

[21] D. Ben-Zvi, B. Shilo, A. Fainsod, N. Barkai. Scaling of the BMP activation gradient in Xenopus embryos. Nature, 453 (2008), 1205-1211.

[22] H. Berg. Random walks in biology 1983.

[23] H. C. Berg. Motile behavior of bacteria. Physics Today, 53 (2000), No. 1, 24-29.

[24] H. C. Berg. http://webmac.rowland.org/labs/bacteria/movies/others/. (2008).

[25] M. D. Betterton, M. P. Brenner. Collapsing bacterial cylinders. Physical Review E, 64 (2001), 061904.

[26] K. Bisset, C. Douglas. A continuous study of morphological phase in the swarm of proteus. J. Med. Microbiol., 9 (1976), 229-31.

[27] V. Bolos, J. Grego-Bessa, J. L. de la Pompa. Notch signaling in development and cancer. Endocr Rev, 28 (2007), No. 3, 339-63.

[28] J. T. Bonner. The development of Dictyostelium, Chapter Comparative Biology of Cellular Slime Molds. Academic Press 1982, 1-33.

[29] R. B. Bourret, K. A. Borkovich, M. I. Simon. Signal transduction pathways involving protein phosphorylation in prokaryotes. 60 (1991), 401-441.

[30] P. Brakefield, V. French. Eyespot development on butterfly wings: the epidermal response to damage. Dev. Biol., 168 (1995), 98-111.

[31] M. P. Brenner, L. S. Levitov, E. O. Budrene. Physical mechanisms for chemotactic pattern formation by bacteria. 74 (1998), No. 4, 1677-1693.

[32] C. Brunetti, J. Selegue, A. Monteiro, V. French, P. Brakefield, S. Carroll. The generation and diversification of butterfly eyespot color patterns. Curr. Biol., 11 (2001), 1578-1585.

[33] E. O. Budrene. Personal communication 2005. 
[34] E. O. Budrene, H. C. Berg. Complex patterns formed by motile cells of Escherichia coli. Nature, 349 (1991), No. 6310, 630-633.

[35] E. O. Budrene, H. C. Berg. Dynamics of formation of symmetrical patterns by chemotactic bacteria. Nature, 376 (1995), No. 6535, 49-53.

[36] C. Caicedo-Carvajal, T. Shinbrot. In silico zebrafish pattern formation. Dev. Biol., 315 (2008), 397-403.

[37] S. Carroll, J. Gates, D. Keys, S. Paddock, G. Panganiban, J. Selegue, J. Williams. Pattern formation and eyespot determination in butterfly wings. Science, 265 (1994), 109-114.

[38] J. Casanova, G. Struhl. The torso receptor localizes as well as transduces the spatial signal specifying terminal body pattern in Drosophila. Nature, 362 (1993), 152-155.

[39] V. Castets, E. Dulos, P. D. Kepper. Experimental evidence of a sustained standing Turingtype nonequilibrium chemical pattern. 64 (1990), No. 24, 2953-2956.

[40] C. M. Child. Patterns and problems of development. University of Chicago Press 1941.

[41] S. Childress, J. K. Percus. Nonlinear aspects of chemotaxis. 56 (1981) 217-237.

[42] J. H. Claxton. The determination of patterns with special reference to that of the central primary skin follicles in sheep. J. Theor. Biol., 7 (1964), 302-317.

[43] J. H. Claxton. Developmental origin of even spacing between the microchaetes of Drosophila melanogaster. Aust J Biol Sci, 29 (1976) 131-135.

[44] P. Cluzel, M. Surette, S. Leibler. An ultrasensitive bacterial motor revealed by monitoring signaling proteins in single cells. Science, 287 (2000), 1652-1655.

[45] E. Conway, D. Hoff, J. Smoller. Large time behavior of solutions of systems of nonlinear reaction-diffusion equations. SIAM J. Appl. Math., (1977).

[46] M. Coppey, A. Berezhkovskii, Y. Kim, A. Boettiger, S. Shvartsman. Modeling the bicoid gradient: diffusion and reversible nuclear trapping of a stable protein. Dev. Biol., 312 (2007), 623-630.

[47] M. Coppey, A. Boettiger, A. Berezhkovskii, S. Shvartsman. Nuclear trapping shapes the terminal gradient in the Drosophila embryo. Curr. Biol., 18 (2008), 915-919.

[48] E. Crampin, W. Hackborn, P. Maini. Pattern formation in reaction-diffusion models with nonuniform domain growth. Bull. Math. Biol., 64 (2002), 747-769.

[49] E. J. Crampin, E. A. Gaffney, P. K. Maini. Reaction and diffusion on growing domains: Scenarios for robust pattern formation. Bulletin of Mathematical Biology, 61 (1999), No. 6, 1093-1120. 
[50] O. Crauk, N. Dostatni. Bicoid determines sharp and precise target gene expression in the Drosophila embryo. Curr Biol, 15 (2005), No. 21, 1888-1898, comparative Study.

[51] F. H. Crick. Diffusion in embryogenesis. Nature, 225 (1970), 420-422.

[52] M. C. Cross, P. C. Hohenberg. Pattern formation out of equilibrium. 65 (1993), No. 3, 8511112 .

[53] R. Daniels, J. Vanderleyden, J. Michiels. Quorum sensing and swarming migration in bacteria. FEMS Microbiol Rev., 28 (2004), 261-89.

[54] P. de Kepper, V. Castets, E. Dulos, J. Boissonade. Turing-type chemical patterns in the chlorite-iodide-malonic acid reaction. D 49 (1991) 161-169.

[55] R. Dilão, J. Sainhas. Modelling butterfly wing eyespot patterns. Proc. Biol. Sci., 271 (2004), 1565-1569.

[56] R. Dillon, P. K. Maini, H. G. Othmer. Pattern formation in generalized Turing systems I. Steady-state patterns in systems with mixed boundary conditions. Journal of Mathematical Biology, 32 (1994), No. 4, 345-393.

[57] R. Dillon, H. G. Othmer. A mathematical model for outgrowth and spatial patterning of the vertebrate limb bud. J. Theor. Biol., 197 (1999), No. 3, 295-330.

[58] W. Driever, C. Nüsslein-Volhard. A gradient of bicoid protein in Drosophila embryos. Cell, 54 (1988), No. 1, 83-93.

[59] W. Driever, C. Nusslein-Volhard. The bicoid protein determines position in the Drosophila embryo in a concentration-dependent manner. Cell, 54 (1988), No. 1, 95-104.

[60] S. Eglen. Development of regular cellular spacing in the retina: theoretical models. Mathematical Medicine and Biology, 23 (2006), No. 2, 79-99.

[61] A. Eldar, R. Dorfman, D. Weiss, H. Ashe, B. Z. Shilo, N. Barkai. Robustness of the BMP morphogen gradient in drosophila embryonic patterning. Nature, 419 (2002), No. 6904, 304-308.

[62] R. Erban. From individual to collective behavior in biological systems. Ph.D. thesis, University of Minnesota 2005.

[63] R. Erban, H. Othmer. From signal transduction to spatial pattern formation in E. coli: A paradigm for multiscale modeling in biology. 3 (2005), No. 2, 362-394.

[64] R. Erban, H. G. Othmer. From individual to collective behavior in bacterial chemotaxis. SIAM J. Appl. Math., 65 (2004), No. 2, 361-391.

[65] R. Erban, H. G. Othmer. Taxis equations for amoeboid cells. J Math Biol, 54 (2007), 847885. 
[66] B. Ermentrout. Stripes or spots? nonlinear effects in bifurcation of reaction-diffusion equations on the square. Proc. Roy. Soc. Lond. A., 434 (1991), 413-417.

[67] T. Evans, J. Marcus. A simulation study of the genetic regulatory hierarchy for butterfly eyespot focus determination. Evol. Dev., 8 (2006) 273-283.

[68] A. Filloux, I. Vallet. Biofilm: set-up and organization of a bacterial community. Med Sci (Paris), 19 (2003), No. 1, 77-83.

[69] P. R. Fisher, R. Merkl, G. Gerisch. Quantitative analysis of cell motility and chemotaxis in Dictyostelium discoideum by using an image processing system and a novel chemotaxis chamber providing stationary chemical gradients. J. Cell. Biol., 108 (1989), 973-984.

[70] R. M. Ford, D. A. Lauffenburger. Analysis of chemotactic bacterial distributions in population migration assays using a mathematical model applicable to steep or shallow attractant gradients. 53 (1991), No. 5, 721-749.

[71] R. M. Ford, D. A. Lauffenburger. Measurement of bacterial random motility and chemotaxis coefficients: II: Application of single-cell-based mathematical model. Biotechnol. Bioeng., 37 (1991), 661-672.

[72] R. M. Ford, B. R. Phillips, J. A. Quinn, D. A. Lauffenburger. Measurement of bacterial random motility and chemotaxis coefficients: I. stopped-flow diffusion chamber assay. Biotechnol. Bioeng., 37 (1991) 647-660.

[73] C. Fowlkes, C. Hendriks, S. KerŁnen, G. Weber, O. Rbel, M. Huang, S. Chatoor, A. DePace, L. Simirenko, C. Henriquez, A. Beaton, R. Weiszmann, S. Celniker, B. Hamann, D. Knowles, M. Biggin, M. Eisen, J. Malik. A quantitative spatiotemporal atlas of gene expression in the Drosophila blastoderm. Cell, 133 (2008), 364-374.

[74] H. Fricke. Juvenile-adult colour patterns and coexistence in the territorial coral reef fish Pomacanthus imperator. Marine Ecology, 1 (1980), 133-141.

[75] A. Gierer, H. Meinhardt. A theory of biological pattern formation. 12 (1972), No. 1, 30-39.

[76] L. Glass. Stochastic generation of regular distributions. Science, 180 (1973), 1061-1063.

[77] L. A. Goentoro, G. T. Reeves, C. P. Kowal, L. Martinelli, T. Schpbach, S. Y. Shvartsman. Quantifying the Gurken morphogen gradient in Drosophila oogenesis. Dev. Cell, 11 (2006), 263-272.

[78] A. B. Goryachev, D. J. Toh, T. Lee. Systems analysis of a quorum sensing network: design constraints imposed by the functional requirements, network topology and kinetic constants. Biosystems, 83 (2006), No. 2-3, 178-87.

[79] C. Graván, R. Lahoz-Beltra. Evolving morphogenetic fields in the zebra skin pattern based on turing's morphogen hypothesis. Int. J. Appl. Math. Comput. Sci., 14 (2004), 351-361. 
[80] E. P. Greenberg. Bacterial communication: Tiny teamwork. Nature, 424 (2003), 134.

[81] T. Gregor, W. Bialek, R. R. de Ruyter van Steveninck, D. W. Tank, E. F. Wieschaus. Diffusion and scaling during early embryonic pattern formation. Proc Natl Acad Sci U S A, 102 (2005), No. 51, 18403-7.

[82] T. Gregor, D. W. Tank, E. F. Wieschaus, W. Bialek. Probing the limits to positional information. Cell, 130 (2007), No. 1, 153-164.

[83] T. Gregor, E. F. Wieschaus, A. P. McGregor, W. Bialek, D. W. Tank. Stability and nuclear dynamics of the bicoid morphogen gradient. Cell, 130 (2007), No. 1, 141-152.

[84] D. Grunwald, J. Eisen. Headwaters of the zebrafish - emergence of a new model vertebrate. Nat. Rev. Genet., 3 (2002), 717-724.

[85] P. Haffter, J. Odenthal, M. C. Mullins, S. Lin, M. J. Farrell, L. Vogelsang, H. F., M. Brand, F. van Eeden, M. Furutani-Seiki, M. Granato, M. Hammerschmidt, C. P. Heisenberg, Y. J. Jiang, D. A. Kane, N. Kelsh, R. N. Hopkins, C. Nusslein-Volhard. Mutationa affecting pigmentation and shape of the adult zebrafish. Dev. Genes. Evol., 206 (1996), 260-276.

[86] H.Berg, D. Brown. Chemotaxis in Escherichia Coli analyzed by three-dimensional tracking. Nature, 239 (1972), 502-507.

[87] D. Headon, K. J. Painter. Stippling the skin: Generation of anatomical periodicity by reaction-diffusion mechanisms. Submitted to MMNP, (2008).

[88] M. Herrero, J. Velázquez. Chemotactic collapse for the Keller-Segel model. J. Math. Biol., 35 (1996), 177-194.

[89] T. Hillen, H. G. Othmer. The diffusion limit of transport equation derived from velocity-jump processes. Siam J. Appl. Math., 61 (2000), No. 3, 751-775.

[90] T. Hillen, K. Painter. A user's guide to pde models for chemotaxis. J. Math. Biol., 58 (2008), 183-217.

[91] M. Hirata, K.-i. Nakamura, T. Kanemaru, Y. Shibata, S. Kondo. Pigment cell organization in the hypodermis of zebrafish. Dev Dyn, 227 (2003) 497-503.

[92] D. Horstmann. From 1970 until present: the Keller-Segel model in chemotaxis and its consequences I. Jahresbericht der DMV, 105 (2003), No. 3, 103-165.

[93] D. Horstmann. A constructive approach to traveling waves in chemotaxis. Journal of Nonlinear Science, 14 (2004), 1-25(25).

[94] B. Houchmandzadeh, E. Wieschaus, S. Leibler. Establishment of developmental precision and proportions in the early Drosophila embryo. Nature, 415 (2002), No. 6873, 798-802. 
[95] L. Hufnagel, A. A. Teleman, H. Rouault, S. M. Cohen, B. I. Shraiman. On the mechanism of wing size determination in fly development. Proc Natl Acad Sci U S A, 104 (2007), No. 10, 3835-40, epub 2007 Feb 28.

[96] J. Jaeger, J. Reinitz. On the dynamic nature of positional information. Bioessays, 28 (2006), No. 11, 1102-11.

[97] J. Jaeger, S. Surkova, M. Blagov, H. Janssens, D. Kosman, K. Kozlov, Manu, E. Myasnikova, C. Vanario-Alonso, M. Samsonova, D. Sharp, J. Reinitz. Dynamic control of positional information in the early Drosophila embryo. Nature, 430 (2004), No. 430, 368-71.

[98] W. Jäger, S. Luckhaus. On explosions of solutions to a system of partial differential equations modelling chemotaxis. 329 (1992), No. 2, 819-824.

[99] S. L. Johnson, D. Africa, C. Walker, J. A. Weston. Genetic control of adult pigment stripe development in zebrafish. Dev Biol, 167 (1995), 27-33.

[100] H. S. Jung, P. H. Francis-West, R. B. Widelitz, T. X. Jiang, S. Ting-Berreth, C. Tickle, L. Wolpert, C. M. Chuong. Local inhibitory action of BMPs and their relationships with activators in feather formation: implications for periodic patterning. Dev Biol, 196 (1998), No. 1, 11-23.

[101] D. Kaiser. Coupling cell movement to multicellular development in myxobacteria. Nat. Rev. Microbiol., 1 (2003), No. 1, 45-54.

[102] D. Kaiser. Myxococcus - from single-cell polarity to complex multicellular patterns. Annual Review of Genetics, 42 (2008), No. 1, 109-130.

[103] E. F. Keller, G. M. Odell. Necessary and sufficient conditions for chemotactic bands. Mathematical Biosciences, 27 (1975), 309-317.

[104] E. F. Keller, L. A. Segel. Initiation of slime mold aggregation viewed as an instability. J. Theor. Biol., 26 (1970), 399-415.

[105] E. F. Keller, L. A. Segel. Model for chemotaxis. J. Theor. Biol., 30 (1971), 225-234.

[106] E. F. Keller, L. A. Segel. Traveling bands of chemotactic bacteria: A theoretical analysis. J. Theor. Biol., 30 (1971), 235-248.

[107] R. N. Kelsh, M. Brand, Y. J. Jiang, C. P. Heisenberg, S. Lin, P. Haffter, J. Odenthal, M. C. Mullins, F. J. van Eeden., M. Furutani-Seiki, M. Granato, M. Hammerschmidt, D. A. Kane, R. M. Warga, D. Beuchle, L. Vogelsang, C. Nusslein-Volhard. Zebrafish pigmentation mutations and the processes of neural crest development. Development, 123 (1996), 369-389.

[108] S.V. Keranen, C.C. Fowlkes, C.L. Luengo Hendriks, D. Sudar, D.W. Knowles, J. Malik, M.D. Biggin. Three-dimensional morphology and gene expression in the Drosophila blastoderm at cellular resolution II: dynamics. Genome Biol. 7 (1006), R124 
[109] M. Kerszberg, L. Wolpert. Mechanisms for positional signalling by morphogen transport: a theoretical study. J. Theor. Biol., 191 (1998), No. 1, 103-114.

[110] A. Kicheva, P. Pantazis, T. Bollenbach, Y. Kalaidzidis, T. Bittig, F. Julicher, M. GonzalezGaitan. Kinetics of morphogen gradient formation. Science, 315 (2007), No. 5811, 521-525.

[111] P. Koch, D. Keys, T. Rocheleau, K. Aronstein, M. Blackburn, S. Carroll, R. ffrench Constant. Regulation of dopa decarboxylase expression during colour pattern formation in wild-type and melanic tiger swallowtail butterflies. Development, 125 (1998), 2303-2313.

[112] R. Kolter, E. P. Greenberg. Microbial sciences: The superficial life of microbes. Nature, 441 (2006), 300-302.

[113] S. Kondo, R. Asai. A reaction-diffusion wave on the skin of the marine angelfish Pomacanthus. Nature, 376 (1995), 675-768.

[114] P. Kulesa, G. Cruywagen, S. Lubkin, P. Maini, J. Sneyd, M. Ferguson, J. Murray. On a model mechanism for the spatial patterning of teeth primordia in the alligator. J. Theor. Biol., 180 (1996), 287-296.

[115] A. D. Lander. Morpheus unbound: reimagining the morphogen gradient. Cell, 128 (2007), No. 2, 245-256.

[116] A. D. Lander, Q. Nie, F. Y. M. Wan. Do morphogen gradients arise by diffusion? Dev. Cell, 2 (2002), No. 6, 785-96.

[117] I. R. Lapidus, R. Schiller. A model for traveling bands of chemotactic bacteria. Biophys J., 22 (1978), No. 1, 1-13.

[118] D. Lauffenburger, C. R. Kennedy, R. Aris. Traveling bands of chemotactic bacteria in the context of population growth. Bulletin of Mathematical Biology, 46 (1984), No. 1, 19-40.

[119] N. Le Douarin, C. Kalcheim. The neural crest. CUP, Cambridge, 2nd edition 1999.

[120] I. Lengyel, I. R. Epstein. Modelling of Turing structures in the chlorite-iodide-malonic acidstarch reaction system. Science, 251 (1991) 650-652.

[121] S. Liaw, C. Yang, R. Liu, J. Hong. Turing model for the patterns of lady beetles. Phys Rev E Stat Nonlin Soft Matter Phys, 64 (2001), 041909.

[122] R. Liu, S. Liaw, P. Maini. Two-stage Turing model for generating pigment patterns on the leopard and the jaguar. Phys Rev E Stat Nonlin Soft Matter Phys, 74 (2006), 011914.

[123] R. Lux, W. Shi. Chemotaxis-guided movements in bacteria. Crit. Rev. Oral. Biol. Med., 15 (2004), No. 4, 207-20.

[124] M. Lyons, L. Harrison. Stripe selection: An intrinsic property of some pattern-forming models with nonlinear dynamics. Dev. Dyn., 195 (1992) 201-215. 
[125] F. Maderspacher, C. Nüsslein-Volhard. Formation of the adult pigment pattern in zebrafish requires leopard and obelix dependent cell interactions. Development, 130 (2003), 34473457.

[126] A. Madzvamuse, P. Maini, A. Wathen, T. Sekimura. A predictive model for color pattern formation in the butterfly wing of papilio dardanus. Hiroshima Math. J., 32 (2002), 325336.

[127] P. K. Maini, K. J. Painter, H. P. C. Nguyen. Spatial pattern formation in chemical and biological systems. J Chem Soc Faraday Trans, 93 (1997), No. 20, 3601-10.

[128] N. V. Mantzaris, S. Webb, H. G. Othmer. Mathematical modeling of tumor-induced angiogenesis. J. Math. Biol., 49 (2004), No. 2, 111-87.

[129] J. Marcus, T. Evans. A simulation study of mutations in the genetic regulatory hierarchy for butterfly eyespot focus determination. BioSystems, 93 (2008), 250-255.

[130] M. McClure. Development and evolution of melanophore patterns in fishes of the genus Danio (Teleostei: Cyprinidae). J. Morphol., 241 (1999) 83-105.

[131] H. Meinhardt. Models of biological pattern formation. Academic Press, New York 1980.

[132] H. Meinhardt. Models for positional signalling with application to the dorsoventral patterning of insects and segregation into different cell types. Development, supplement (1989), 169-180.

[133] H. Meinhardt, P. Prusinkiewicz, D. Fowler. The algorithmic beauty of sea shells. Springer 2003.

[134] B. A. Mello, Y. Tu. Quantitative modeling of sensitivity in bacterial chemotaxis: the role of coupling among different chemoreceptor species. Proc. Nat. Acad. Sci. (USA), 100 (2003), 8223-8228.

[135] D. Míguez, A. Muñuzuri. On the orientation of stripes in fish skin patterning. Biophys. Chem., 124 (2006), 161-167.

[136] N. Milos, A. D. Dingle. Dynamics of pigment pattern formation in the zebrafish, Brachydanio rerio. I. Establishment and regulation of the lateral line melanophore stripe during the first eight days of development. J Exp Zool, 205 (1978), 205-216.

[137] N. Mittal, E. O. Budrene, M. P. Brenner, A. Oudenaarden. Motility of Escherichia coli cells in clusters formed by chemotactic aggregation. Proc. Natl. Acad. Sci. (USA), 100 (2003), No. 23, 13259-63.

[138] C. M. Mizutani, Q. Nie, F. Y. Wan, Y. T. Zhang, P. Vilmos, R. Sousa-Neves, E. Bier, J. L. Marsh, A. D. Lander. Formation of the BMP activity gradient in the Drosophila embryo. Dev. Cell, 8 (2005), No. 6, 915-24. 
[139] A. Monteiro, V. French, G. Smit, P. Brakefield, J. Metz. Butterfly eyespot patterns: evidence for specification by a morphogen diffusion gradient. Acta Biotheor., 49 (2001), 77-88.

[140] J. R. Mooney, B. N. Nagorcka. Spatial patterns produced by a reaction-diffusion system in primary hair follicles. J. Theor. Biol., 115 (1985), 299-317.

[141] J. Moreira, A. Deutsch. Pigment pattern formation in zebrafish during late larval stages: a model based on local interactions. Dev. Dyn., 232 (2005).

[142] C. Mou, B. Jackson, P. Schneider, P. A. Overbeek, D. J. Headon. Generation of the primary hair follicle pattern. Proc. Natl. Acad. Sci. U.S.A., 103 (2006), 9075-9080.

[143] J. D. Murray. A pattern formation mechanism and its application to mammalian coat markings. volume 39 of Lecture Notes in Biomathematics, Springer, Berlin, Heidelberg, New York. 1979, (360-399).

[144] J. D. Murray. On pattern-formation mechanisms for leipdopteran wing patterns and mammalian coat markings. Phil. Trans. Roy. Soc. Lond. B, 295 (1981), 473-496.

[145] J. D. Murray. A pre-pattern formation mechanism for animal coat markings. J. Theor. Biol., 88 (1981), 161-199.

[146] J. D. Murray. Mathematical biology ii: Spatial models and biomedical applications. Springer, New York, 3rd edition 2003.

[147] J. D. Murray, D. Deeming, M. Ferguson. Size-dependent pigmentation-pattern formation in embryos of alligator-mississippiensis - time of initiation of pattern generation mechanism. Proc. Roy. Soc. Lond. B, 239 (1990), 279-293.

[148] J. D. Murray, M. Myerscough. Pigmentation pattern formation on snakes. J. Theor. Biol., 149 (1991), 339-360.

[149] J. D. Murray. Mathematical biology, volume 19 of Biomathematics 1989.

[150] B. N. Nagorcka, J. R. Mooney. The role of a reaction-diffusion system in the formation of hair fibres. J. Theor. Biol., 98 (1982) 575-607.

[151] B. N. Nagorcka, J. R. Mooney. The role of a reaction-diffusion system in the initiation of primary hair follicles. J. Theor. Biol., 114 (1985), 243-272.

[152] T. Naitoh, A. Morioka, Y. Omura. Adaptation of a common freshwater goby, yoshinobori, rhinogobius brunneus temminck et schlegel to various backgrounds including those containing different sizes of black and white checkerboard squares. Zool. Sci., 2 (1985), 59.

[153] J. Nelson. Fishes of the world. John Wiley and Sons, New York, 3rd edition 1993. 
[154] F. S. Neuman-Silberberg, T. Schupbach. The Drosophila dorsoventral patterning gene gurken produces a dorsally localized RNA and encodes a TGF alpha-like protein. Cell, 75 (1993), No. 1, 165-74.

[155] H. Nijhout. Wing pattern formation in lepidoptera: a model. J. Exp. Zool., 206 (1978), 119-136.

[156] H. Nijhout. A comprehensive model for color pattern formation in butterflies. Proc. Roy. Soc. Lond. B, 239 (1990), 81-113.

[157] H. Nijhout, P. K. Maini, A. Madzvamuse, A. Wathen, T. Sekimura. Pigmentation pattern formation in butterflies: experiments and models. C. R. Biol., 326 (2003), 717-727.

[158] M. B. O'Connor, D. M. Umulis, H. G. Othmer, S. S. Blair. Shaping BMP morphogen gradients in the Drosophila embryo and pupal wing. Development, 133 (2006), 183-93.

[159] J. Odenthal, K. Rossnagel, P. Haffter, R. N. Kelsh, E. Vogelsang, M. Brand, F. J. van Eeden., M. Furutani-Seiki, M. Granato, M. Hammerschmidt, C. P. Heisenberg, Y. J. Jiang, D. A. Kane, M. C. Mullins, C. Nusslein-Volhard. Mutations affecting xanthophore pigmentation in the zebrafish, Danio rerio. Development, 123 (1996), 391-398.

[160] J. Otaki. Physiologically induced color-pattern changes in butterfly wings: mechanistic and evolutionary implications. J. Insect Physiol., 54 (2008), 1099-1112.

[161] H. G. Othmer. Interactions of reaction and diffusion in open systems. Ph.D. thesis, University of Minnesota, Minneapolis 1969.

[162] H. G. Othmer. Current problems in pattern formation. In Some mathematical questions in biology, volume VIII, Amer. Math. Soc., Providence, R.I. 1977, (57-85).

[163] H. G. Othmer. Synchronized and differentiated modes of cellular dynamics. In H. Haken, editor, Dynamics of Synergetic Systems, Springer-Verlag.

[164] H. G. Othmer, J. A. Aldridge. The effects of cell density and metabolite flux on cellular dynamics. J. Math. Biol., 5 (1978), 169-200.

[165] H. G. Othmer, S. R. Dunbar, W. Alt. Models of dispersal in biological systems. J. Math. Biol., 26 (1988), No. 3, 263-298.

[166] H. G. Othmer, T. Hillen. The diffusion limit of transport equations, Part II: chemotaxis equations. SIAM JAM, 62 (2002), 1222-1260.

[167] H. G. Othmer, E. F. Pate. Scale-invariance in reaction-diffusion models of spatial pattern formation. Proc Natl Acad Sci U S A, 77 (1980), No. 7, 4180-4184.

[168] H. G. Othmer, P. Schaap. Oscillatory cAMP signaling in the development of Dictyostelium discoideum. Comments on Theoretical Biology, 5 (1998), 175-282. 
[169] H. G. Othmer, L. E. Scriven. Instability and dynamic pattern in cellular networks. J. Theor. Biol., 32 (1971), 507-537.

[170] H. G. Othmer, A. Stevens. Aggregation, blowup and collaps: The ABC's of generalized taxis. SIAM J. Appl. Math., 57 (1997), No. 4, 1044-1081.

[171] Q. Ouyang, H. L. Swinney. Transition from a uniform state to hexagonal and striped turing patterns. Nature, 352 (1991), 610-612.

[172] K. Painter. Mathematical models for biological pattern formation, chapter Modelling of pigment patterns in fish. Number 121 in IMA Volumes in Mathematics and its Applications, Springer-Verlag, Berlin 2000, (59-82).

[173] K. J. Painter, P. K. Maini, H. G. Othmer. Stripe formation in juvenile Pomacanthus explained by a generalized Turing mechanism with chemotaxis. Proc. Nat. Acad. Sci., 96 (1999), 5549-5554.

[174] K. J. Painter, P. K. Maini, H. G. Othmer. Development and applications of a model for cellular response to multiple chemotactic cues. J. Math. Biol., 41 (2000), No. 4, 285-314.

[175] D. Parichy. Homology and the evolution of novelty during Danio adult pigment pattern development. J. Exp. Zool. B Mol. Dev. Evol., 308 (2007), 578-590.

[176] D. M. Parichy, D. G. Ransom, B. Paw, L. I. Zon, S. L. Johnson. An orthologue of the kit-related gene fms is required for development of neural crest-derived xanthophores and a subpopulation of adult melanocytes in the zebrafish, Danio rerio. Development, 127 (2000), 3031-3044.

[177] D. M. Parichy, J. M. Turner. Temporal and cellular requirements for Fms signaling during zebrafish adult pigment pattern development. Development, 130 (2003), 817-833.

[178] D. M. Parichy, J. M. Turner. Zebrafish puma mutant decouples pigment pattern and somatic metamorphosis. Dev Biol, 256 (2003), 242-257.

[179] D. M. Parichy, J. M. Turner, N. B. Parker. Essential role for puma in development of postembryonic neural crest-derived cell lineages in zebrafish. Dev Biol, 256 (2003), 221-241.

[180] E. Pate, H. G. Othmer. Differentiation, cell sorting and proportion regulation in the slug stage of Dictyostelium discoideum. J. Theor. Biol., 118 (1986), No. 3, 301-319.

[181] C. S. Patlak. Random walk with persistence and external bias. Bull. of Math. Biophys., 15 (1953), 311-338.

[182] J. E. Pearson, W. Horsthemke. Turing instabilities with nearly equal diffusion coefficients. Journal of Chemical Physics, 90 (1989), No. 3, 1588-1599. 
[183] F. Peri. The role of EGF and TGF-b signaling in specifying the polarity of the Drosophila egg and embryo. Doctoral, University of Cologne 2001.

[184] T. J. Perkins, J. Jaeger, J. Reinitz, L. Glass. Reverse engineering the gap gene network of Drosophila melanogaster. PLoS Comput Biol, 2 (2006), No. 5, 0417-28.

[185] A. A. Polezhaev, R. A. Pashkov, A. I. Lobanov, I. B. Petrov. Spatial patterns formed by chemotactic bacteria escherichia coli. Int J Dev Biol, 50 (2006), No. 2-3, 309-14.

[186] R. Prum, R. Torres. Structural colouration of mammalian skin: convergent evolution of coherently scattering dermal collagen arrays. J. Exp. Biol., 207 (2004), 2157-2172.

[187] R. Prum, S. Williamson. Reaction-diffusion models of within-feather pigmentation patterning. Proc. Biol. Sci., 269 (2002), 781-792.

[188] C. V. Rao, J. R. Kirby, A. P. Arkin. Design and diversity in bacterial chemotaxis: A comparative study in Escherichia coli and Bacillus subtilis. PLoS Biol, 2 (2004), No. 2, E49.

[189] O. Rauprich, M. Matsushita, C. J. Weijer, F. Siegert, S. E. Esipov, J. A. Shapiro. Periodic phenomena in proteus mirabilis swarm colony development. J Bacteriol, 178 (1996), No. 22, $6525-38$.

[190] G. Reeves, R. Kalifa, D. Klein, M. Lemmon, S. Shvartsman. Computational analysis of EGFR inhibition by Argos. Dev. Biol., 284 (2005), 523-535.

[191] G. T. Reeves, C. B. Muratov, T. Schupbach, S. Y. Shvartsman. Quantitative models of developmental pattern formation. Dev Cell, 11 (2006), No. 3, 289-300.

[192] T. Roose, S. J. Chapman, P. K. Maini. Mathematical models of avascular tumor growth. SIAM Review, 49 (2007), No. 2, 179-208.

[193] G. Ruxton. The possible fitness benefits of striped coat coloration for zebra. Mammal. Rev., 32 (2002), 237-244.

[194] M. J. Saxton. A Biological Interpretation of Transient Anomalous Subdiffusion. I. Qualitative Model. Biophysical Journal, 92 (2007), No. 4, 1178.

[195] H. Scher, M. Lax. Stochastic transport in a disordered solid. I. theory. Phys. Rev. B, 7 (1973), No. 10, 4491-502.

[196] B. Schwanwitsch. On the groundplan of the wing pattern in nymphalids and certain other families of rhopalocara. Proc. Zool. Sci. Lond., 34 (1924) 509-528.

[197] J. E. Segall, S. M. Block, H. C. Berg. Temporal comparisons in bacterial chemotaxis. Proc. Nat. Acad. Sci. USA, 83 (1986), 8987-8991.

[198] L. A. Segel. A theoretical study of receptor mechanisms in bacterial chemotaxis. SIAM Journal on Applied Mathematics, 32 (1977), 653-665. 
[199] T. Sekimura, A. Madzvamuse, A. Wathen, P. K. Maini. A model for colour pattern formation in the butterfly wing of Papilio dardanus. Proc. Biol. Sci., 267 (2000), 851-859.

[200] M. Serpe, D. Umulis, A. Ralston, J. Chen, D. Olson, A. Avanesov, H. Othmer, M. O'Connor, S. Blair. The BMP-binding protein Crossveinless 2 is a short-range, concentrationdependent, biphasic modulator of BMP signaling in Drosophila. Dev. Cell, 14 (2008) 940953.

[201] J. A. Shapiro. Thinking about bacterial populations as multicellular organisms. Ann Rev Microbiol, 52 (1998), 81-104.

[202] T. S. Shimizu, S. V. Aksenov, D. Bray. A spatially extended stochastic model of the bacterial chemotaxis signalling pathway. J. Mol. Biol., 329 (2003), 291-309.

[203] O. Shimmi, D. Umulis, H. G. Othmer, M. B. O'Connor. Facilitated transport of a Dpp/Scw heterodimer by Sog/Tsg leads to robust patterning of the Drosophila blastoderm embryo. Cell, 120 (2005), No. 6, 873-86.

[204] H. Shoji, Y. Iwasa, A. Mochizuki, S. Kondo. Directionality of stripes formed by anisotropic reaction-diffusion models. J. Theor. Biol., 214 (2002), 549-561.

[205] H. Shoji, A. Mochizuki, Y. Iwasa, M. Hirata, T. Watanabe, S. Hioki, S. Kondo. Origin of directionality in the fish stripe pattern. Dev. Dyn., 226 (2003), 627-633.

[206] S. Y. Shvartsman, C. B. Muratov, D. A. Lauffenburger. Modeling and computational analysis of EGF receptor-mediated cell communication in Drosophila oogenesis. Development, 129 (2002), 2577-2589.

[207] S. Sick, S. Reinker, J. Timmer, T. Schlake. WNT and DKK determine hair follicle spacing through a reaction-diffusion mechanism. Science, 314 (2006), No. 5804, 1447-50.

[208] D. Silver, L. Hou, W. Pavan. The genetic regulation of pigment cell development. Adv. Exp. Med. Biol., 589 (2006), 155-169.

[209] R. Singh, D. Paul, R. K. Jain. Biofilms: implications in bioremediation. J. Math. Biol., 14 (2006), No. 9, 389-97.

[210] P. A. Spiro, J. S. Parkinson, H. G. Othmer. A model of excitation and adaptation in bacterial chemotaxis. PNAS., 94 (1997), No. 14, 7263-7268.

[211] A. Spirov, K. Fahmy, M. Schneider, E. Frei, M. Noll, S. Baumgartner. Formation of the bicoid morphogen gradient: an mRNA gradient dictates the protein gradient. Development., 136 (2009), 605-614.

[212] M. Steinberg. Differential adhesion in morphogenesis: a modern view. Curr. Opin. Genet. Dev., 17 (2007), 281-286. 
[213] F. Süffert. Die ausbildung des imaginalen flügelschnittes in der schmetterlingspuppe. Z. Morph. Ökol. Tiere, 14 (1929), 338-359.

[214] M. Sugimoto. Morphological colour changes in the medaka, oryzias latipes, after prolonged background adaptation - $i$. changes in the population and morphology of the melanophores. Comp. Biochem. Physiol., 104A (1993), 513.

[215] M. Sugimoto. Morphological color changes in fish: regulation of pigment cell density and morphology. Microsc. Res. Tech., 58 (2002) 496-503.

[216] K. Tosney. Long-distance cue from emerging dermis stimulates neural crest melanoblast migration. Dev. Dyn., 229 (2004), 99-108.

[217] P. Trainor. Specification of neural crest cell formation and migration in mouse embryos. Semin. Cell Dev. Biol., 16 (2005), 683-693.

[218] L. Tsimring, H. Levine, I. Aranson, E. Ben-Jacob, I. Cohen, O. Shochet, W. N. Reynolds. Aggregation patterns in stressed bacteria. Phys. Rev. Letts, 75 (1995), No. 9, 1859-1862.

[219] A. M. Turing. The chemical basis of morphogenesis. Phil. Trans. R. Soc. London, 237 (1952), 37-72.

[220] R. Tyson, S. Lubkin, J. Murray. Model and analysis of chemotactic bacterial patterns in a liquid medium. J Math Biol, 38 (1999), 359-375.

[221] R. Tyson, S. R. Lubkin, J. D. Murray. A minimal mechanism for bacterial pattern formation. Proc. R. Soc. Lond. B, 266 (1999), 299-304.

[222] R. Tyson, L. Stern, R. LeVeque. Fractional step methods applied to a chemotaxis model. J. Math. Biol., 41 (2000), 455-75.

[223] D. Umulis, M. O'Connor, H. Othmer. Robustness of embryonic spatial patterning in Drosophila melanogaster. Curr. Top. Dev. Biol., 81 (2008) 65-111.

[224] D. M. Umulis, M. Serpe, M. B. O'Connor, H. G. Othmer. Robust, bistable patterning of the dorsal surface of the Drosophila embryo. Proc Natl Acad Sci U S A, 103 (2006), No. 31, 11613-8.

[225] C. Varea, J. L. Aragon, R. A. Barrio. Confined turing patterns in growing systems. Phys. Rev. E., 56 (1997), 1250-1253.

[226] B. J. Varnum-Finney, E. Voss, D. R. Soll. Frequency and orientation of pseudopod formation of Dictyostelium discoideum amebae chemotaxing in a spatial gradient: Further evidence for a temporal mechanism. 8 (1987), No. 1, 18-26.

[227] G. von Dassow, E. Meir, E. M. Munro, G. M. Odell. The segment polarity network is a robust developmental module. Nature, 406 (2000), No. 6792, 188-92. 
[228] G. H. Wadhams, J. P. Armitage. Making sense of it all: bacterial chemotaxis. Nat. Rev. Mol. Cell Biol., 5 (2004), No. 12, 1024-37.

[229] M. Walters, V. Sperandio. Quorum sensing in Escherichia coli and Salmonella. Int J Med Microbiol., 296 (2006), No. 2-3, 125-31.

[230] X. Wang, R. Harris, L. Bayston, H. Ashe. Type IV collagens regulate BMP signalling in Drosophila. Nature, 455 (2008), 72-77.

[231] Y. C. Wang, E. L. Ferguson. Spatial bistability of Dpp-receptor interactions during Drosophila dorsal-ventral patterning. Nature, 434 (2005), No. 7030, 229-34.

[232] M. P. Weir, C. W. Lo. Gap-junctional communication. compartments in the Drosophila wing imaginal disc. Developmental Biology, 102 (1984) 130-146.

[233] L. Werdelin, L. Olsson. How the leopard got its spots: a phylogenetic view of the evolution of felid coat patterns. Biol. J. Linn. Soc., 62 (1997) 383-400.

[234] N. A. Whitehead, A. M. Barnard, H. Slater, N. J. Simpson, G. P. Salmond. Quorum-sensing in gram-negative bacteria. FEMS Microbiol Rev., 25 (2001), No. 4, 365-404.

[235] L. Wolpert. Positional information and the spatial pattern of cellular differentiation. J. Theor. Biol., 25 (1969), No. 1, 1-67.

[236] L. Wolpert. Positional information and pattern formation. Curr. Topics in Dev. Biol., 6 (1971), 183-224.

[237] D. E. Woodward, R. Tyson, M. R. Myerscough, J. D. Murray, E. O. Budrene, H. C. Berg. Spatio-temporal patterns generated by salmonella typhimurium. Biophys. J., 68 (1995), No. 5, 2181-2189.

[238] C. Xue. Mathematical models of taxis-driven bacterial pattern formation. Ph.D. thesis, University of Minnesota 2008.

[239] C. Xue, H. G. Othmer. Radial and spiral streams formation in bacterium Proteus mirabilis colonies 2009, preprint.

[240] C. Xue, H. G. Othmer. Multiscale models of taxis-driven patterning in bacterial populations. SIAM J. Appl. Math., to appear (2009).

[241] N. Yakoby, C. A. Bristow, I. Gouzman, M. P. Rossi, Y. Gogotsi, T. Schpbach, S. Y. Shvartsman. Systems-level questions in Drosophila oogenesis. Syst Biol (Stevenage), 152 (2005), 276-284.

[242] M. Yamaguchi, E. Yoshimoto, S. Kondo. Pattern regulation in the stripe of zebrafish suggests an underlying dynamic and autonomous mechanism. Proc. Natl. Acad. Sci. U.S.A., 104 (2007), 4790-4793. 
H. G. Othmer et al. The intersection of theory and application in developmental biology

[243] D. Young. A local activator-inhibitor model of vertebrate skin patterns. Math. Biosci., 72 (1984), 51-58.

[244] Y. Zhang, A. Lander, Q. Nie. Computational analysis of BMP gradients in dorsal-ventral patterning of the zebrafish embryo. J. Theor. Biol., 248 (2007), 579-589. 